Escuela Técnica Superior de Ingenieros de Telecomunicación

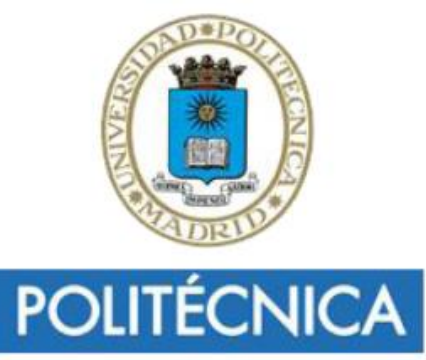

\author{
PhD DISSERTATION
}

\title{
CONTRIBUTION TO SERVICE PROVISION ON INTEROPERABLE MOBILE DEVICES IN CYBER-PHYSICAL SYSTEMS
}

\author{
Author \\ Diego Sánchez de Rivera Córdoba
}

MSc, Telecommunications Engineer

Advisors

Ramón Pablo Alcarria Garrido

Associate professor in Department of Geospatial Engineering.

Universidad Politécnica de Madrid (UPM)

\section{Diego Martín de Andrés}

Associate professor in Department of Telematics Engineering.

Universidad Politécnica de Madrid (UPM)

June 2019 



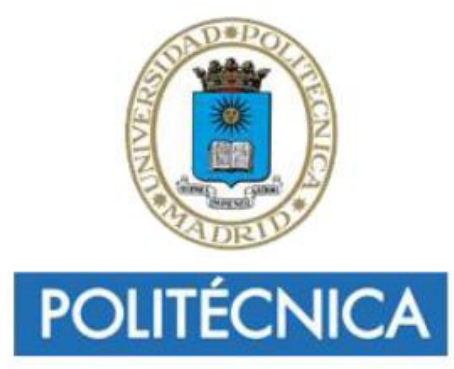

Título de la Tesis: CONTRIBUTION TO SERVICE PROVISION ON INTEROPERABLE MOBILE DEVICES IN CYBER-PHYSICAL SYSTEMS

Departamento: Departamento de Ingeniería de Sistemas Telemáticos

Autor: Diego Sánchez de Rivera Córdoba

Directores: Ramón Pablo Alcarria Garrido

Diego Martín de Andrés

Tribunal nombrado por el Excmo. y Magfco. Sr. Rector de la Universidad Politécnica de Madrid, el día de de

\section{Presidente:}

Vocal:

Vocal:

Vocal:

Secretario:

Suplente:

Suplente:

Realizado el acto de defensa y lectura de Tesis el día Ingenieros de Telecomunicación de Madrid.

de junio de 2019 en la E.T.S. de

\section{Calificación:}

EL PRESIDENTE

LOS VOCALES

EL SECRETARIO 
A Zaira.

Gracias por ser como eres. 


\section{Agradecimientos}

Parecía lejos este momento, cuando hace unos años me embarqué, casi sin saber dónde me metía, en la aventura de realizar un Doctorado. La decisión fue fácil, ya que siempre encontré un gran apoyo de personas, que por un motivo u otro llegaron a mi vida, y a las que dedico estas palabras.

En primer lugar, me gustaría agradecer a Tomás Robles, director del Grupo de Investigación GISAI en el que he podido realizar esta Tesis, su implicación plena, tanto personal como profesional, en el desarrollo de la misma. Sin duda, sus conocimientos han servido para impulsar en muchos momentos el progreso necesario con el que completar este trabajo.

Gran parte de esta motivación se la debo a mis dos directores de Tesis: Ramón y Diego, de los cuales me llevo su profunda vocación por enseñar todo lo que saben, de la mejor forma que puedan hacerlo. Su interés en guiarme por todas las fases de esta Tesis ha sido enrome, y considero que, de no ser por ellos este desafío no hubiera sido tan interesante.

Ellos también forman parte del componente fundamental al trabajar en equipo: las personas. Y es que, junto con Álvaro y Borja, el día a día vivido en el grupo no ha podido ser mejor. Grandes recuerdos me llevo de todos estos años, y seguro que muchos más seguiremos formando.

Si hay personas a quien tengo que agradecer, esas son mis padres. Estas palabras se quedan cortas para plasmar el infinito apoyo que he recibido de ellos, y que con orgullo puedo reconocer, muchas gracias. Incluyo en estas líneas a mi hermano, David, que aun iniciando nuevas aventuras y por muy lejos que se encuentre, siempre encuentro en él su gran generosidad. A mis abuelas, mis tíos, mis primas, y a toda mi familia y amigos, gracias.

A ti, Zaira, por convertirte, junto a mi familia, en la persona más importante de mi vida. Por tu apoyo, por tu paciencia, por estar siempre ahí, por conocerme, por creer en mí, y por muchísimas cosas más, gracias. Gracias por estar a mi lado.

Madrid. Junio de 2019 


\section{Keywords}

cyber physical systems, service provision, interoperable devices, unattended systems, system monitoring, auto-provision deployment, communication efficiency. 


\section{Abstract}

This PhD Thesis is framed under the Cyber Physical System (CPS) concept, and contributes to the provision, process and optimization of the service provision in smart environments. The smart environment model promotes the creation of interoperable relationships between all the elements present in the network. As everyday objects in our lives are continuously incorporating smart capabilities, an appropriate system can improve the end-user experience.

The overall goal of this PhD Thesis work is to design and validate a service provision environment for CPS with smart devices that improve the functionalities and interoperable capabilities of the service environment. Based on a service environment for CPS, several particularities of smart devices and services adapted to specific conditions will be provided.

One of the current top trends in the smart environment paradigm is the serviceoriented system, which frameworks the interaction between the physical infrastructure of the platform and the service offered to the final users. Applications can be then built on top of the layers that manage the smart system in seamless user experience. In this manner, these services may be used to work in applications deployed by technological experts, or might be included in a "prosumer" environment where non-expert users can design, build and deploy their own services in a dynamic way. Additionally, these deployments are often located in distant locations and supported by the addition of smart environment features.

This flexible paradigm offers the capability of being adaptable to each application, but even so, several problems exist that may decrease the reliability and the advantages of the smart systems in real world deployments.

With this work, I contribute to service provision by defining a process that supports the configuration and provision of the system in an automated form, by including a domain expert at the primary phase and a supporting architecture to interact and manage the system.

Service provision is then decomposed and focused in several aspects where I propose to include mechanisms and procedures to improve the user experience in the whole system, based on CPS constraints, and trying to achieve an extensible system framework that can be adapted to the future trends needs.

Furthermore, I propose the addition of several supporting elements to the basic CPS architecture in order to optimize the resource allocation, monitoring process and communication efficiency of a deployed system. These contributions allow the system to be aware of the changes of the infrastructure and provide a dynamic adaptation of the system resources, battery utilization and device capabilities changes that can emerge in a running smart environment. 
To validate the contributions, experimental measures based on simulated and real scenarios have been conducted. The solutions have been verified and relevant conclusions have been extracted. 


\section{Resumen}

Esta tesis doctoral se enmarca dentro del concepto llamado Sistema Ciber-Físico (CPS en inglés) y contribuye a la provisión, el procesado y la optimización de lo relativo a la provisión de servicios en entornos inteligentes. El concepto de entorno inteligente promueve la creación de relaciones fundamentalmente interoperables entre todos los elementos presentes en la red. Dado que, por ejemplo, muchos objetos cotidianos en nuestras vidas incorporan continuamente nuevas capacidades inteligentes, es aquí donde un sistema apropiado puede mejorar la experiencia de los usuarios finales.

El objetivo principal de esta Tesis Doctoral es diseñar y validar un entorno de provisión de servicios para CPS con dispositivos inteligentes que mejoren las funcionalidades y las capacidades de interoperabilidad del entorno. Sobre la base de un entorno de servicios para CPS, se proporcionarán varios detalles de los dispositivos y servicios inteligentes adecuados a las condiciones específicas estudiadas.

Una de las principales tendencias actuales en el paradigma de entorno inteligentes es la orientación a servicios del sistema, el cual, enmarca la interacción entre la infraestructura física de la plataforma y el servicio ofrecido a los usuarios finales. Sobre esto, las aplicaciones pueden construir capas que administran el sistema inteligente con una experiencia de usuario mejorada. Para lograr este objetivo, estos servicios pueden ser implementados para su utilización por expertos tecnológicos o pueden incluirse en un entorno "prosumer" donde los usuarios no expertos puedan diseñar, construir, e implementar sus propios servicios de una manera dinámica. Además, estas implementaciones a menudo se ubican en ubicaciones distantes y pueden ser adaptadas mediante la adición de nuevas características al entorno inteligente.

Este paradigma es tan flexible que ofrece la capacidad de poderse adaptar a cada aplicación, aun así, existen varios problemas que pueden disminuir la confiabilidad y las ventajas de estos sistemas en los despliegues reales.

De esta manera, con este trabajo contribuyo a la provisión de servicios definiendo procesos que permiten la configuración y despliegue del sistema de forma automatizada incluyendo un experto del dominio en la primera fase y una infraestructura que soporte estas interacciones y la administración del sistema.

Esta provisión automatizada de servicios se descompone en profundidad y se concentra en mejorar varias funcionalidades, donde propongo incluir mecanismos y procedimientos para mejorar la experiencia del usuario en todo el sistema. Todo ello basado en las restricciones que impone un CPS y para tratar de lograr un marco de sistema extensible que pueda adaptarse a las necesidades futuras.

Además, propongo añadir varios elementos de soporte a esta arquitectura de manera que se optimice la asignación de recursos, el proceso de monitorización y la eficiencia de las comunicaciones del sistema. Estas contribuciones permiten que el sistema sea 
consciente de los cambios de la infraestructura que pueden surgir en un entorno inteligente en ejecución y proporcione una adaptación dinámica a los recursos del sistema, una mejor utilización de la energía disponible y mejora en las capacidades del dispositivo.

Para validar estas contribuciones, se han realizado medidas experimentales basadas en escenarios tanto simulados como reales. La solución ha sido verificada y se han podido extraer conclusiones relevantes. 


\section{Contents}

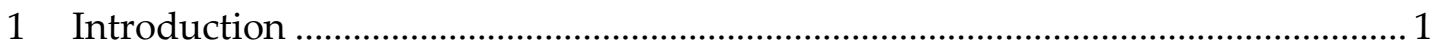

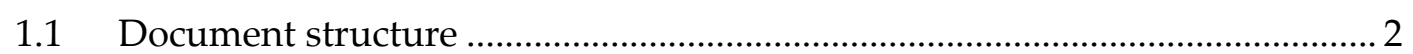

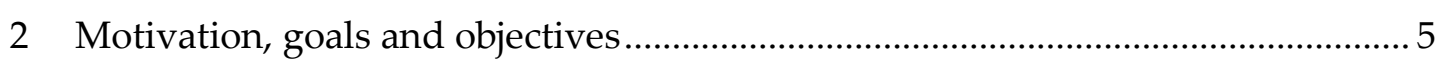

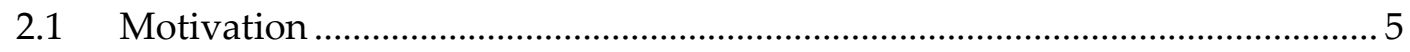

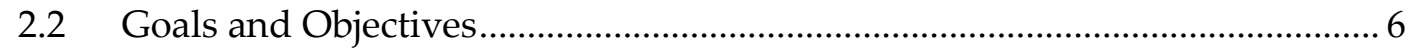

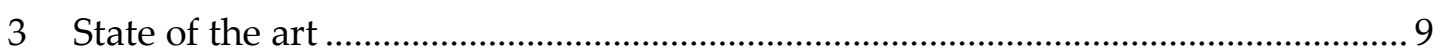

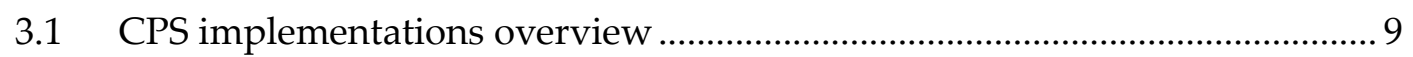

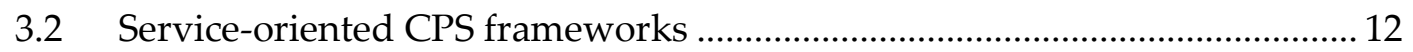

3.3 Service based CPS configuration and adaptation backgrounds ..................... 17

3.4 Automated service distribution at location level............................................ 19

3.5 Considerations on CPS end point devices ….................................................. 21

4 Service provision in unattended systems, methods and requirements ................ 25

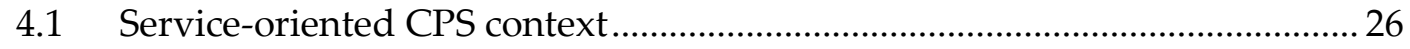

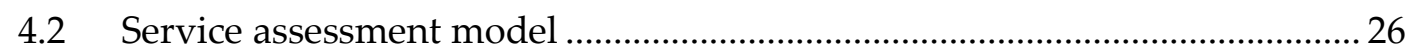

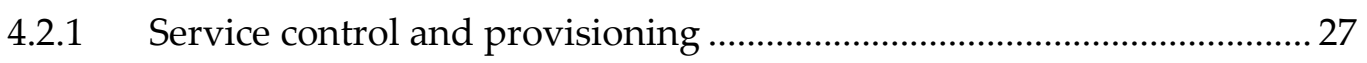

4.2.2 Service data components .......................................................................... 27

4.2.3 Integrating a service generating process ................................................ 30

4.3 Requirement gathering among real deployments .......................................... 33

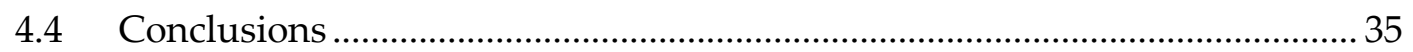

5 Model and reference architecture for the supporting elements ............................ 37

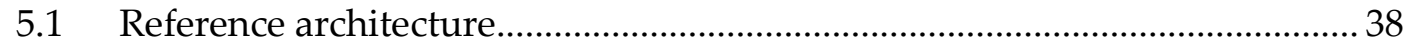

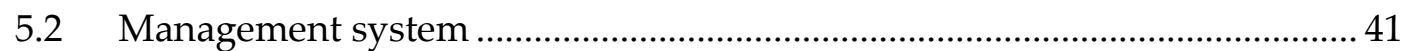

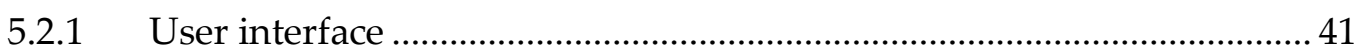

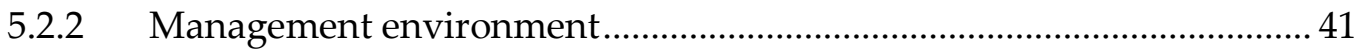

5.2.3 Supporting Facilities. The common Bus.................................................... 43

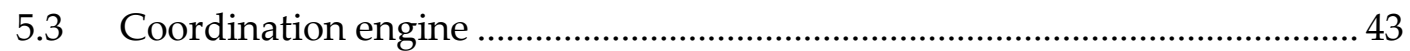

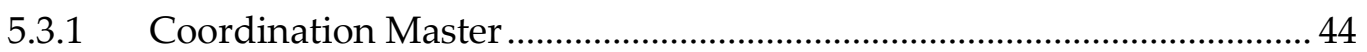

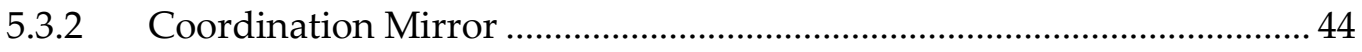

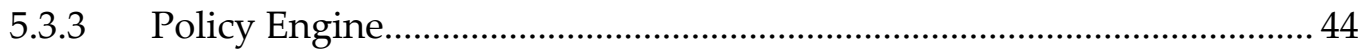

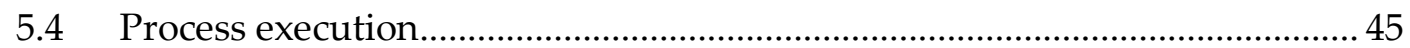

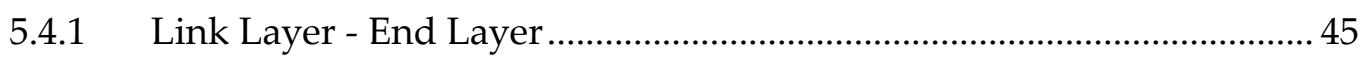




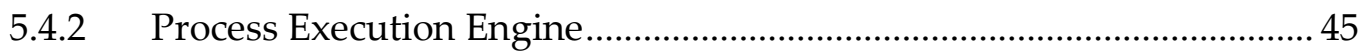

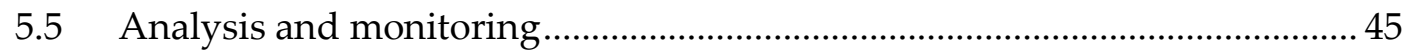

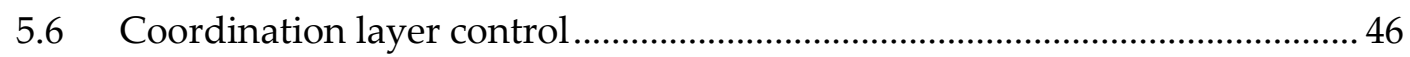

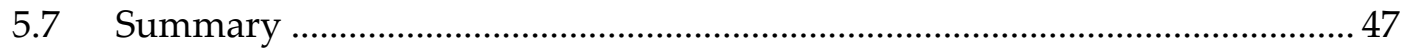

6 Methods and functions to auto-provision deployments ...................................... 49

6.1 Service-based analysis of smart environments ................................................ 50

6.2 Fast-self configuration for interoperable device integration .......................... 52

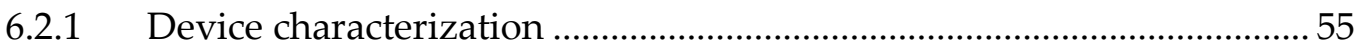

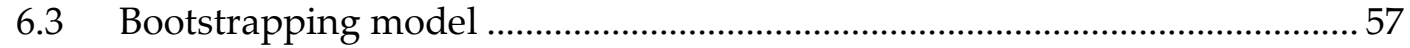

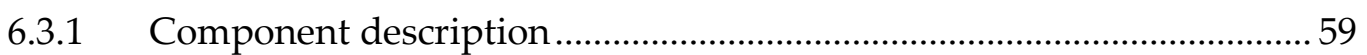

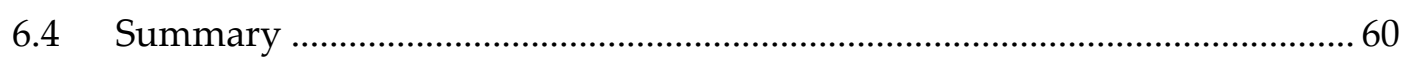

7 Monitor and execution validation model in an interoperable multi-site CPS..... 63

7.1 Monitoring model and process lifecycle definition ..........................................64

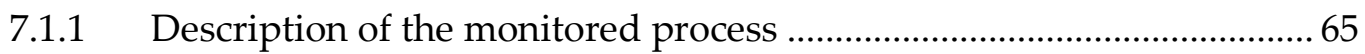

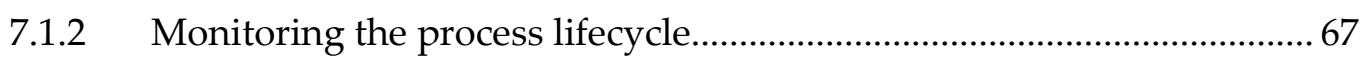

7.2 Distributed CPS monitoring, a resiliency focused approach ..........................68

7.2.1 Benefits and drawbacks of the proposed approach ................................ 70

7.3 A monitoring model for a distributed CPS ....................................................... 70

7.4 Supporting the process execution monitoring …............................................ 73

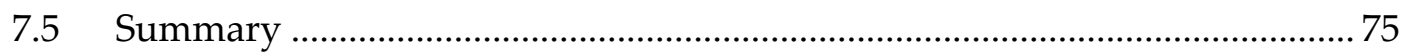

8 Optimizing communication efficiency in constrained endpoint devices............. 77

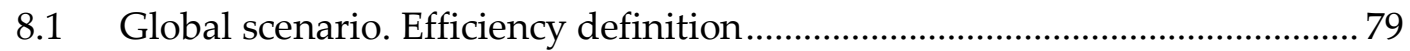

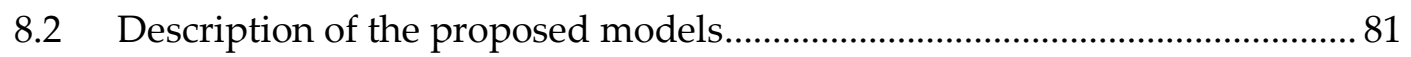

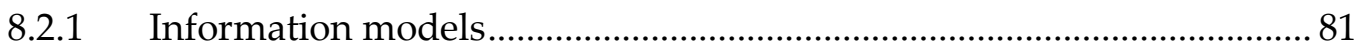

8.2.2 Endpoint device and server models ........................................................ 83

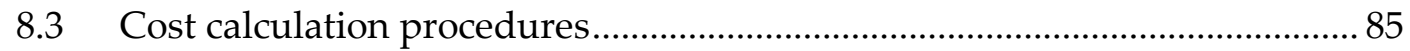

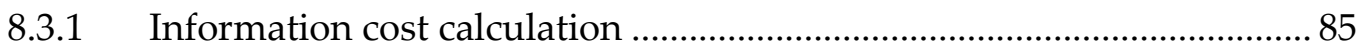

8.3.2 Link management cost calculation........................................................... 88

8.3.3 Information obtention cost calculation.................................................... 90

8.3.4 Information consumption cost calculation ……………………….......... 92

8.4 Proposed algorithm for optimizing communication efficiency …….............. 95

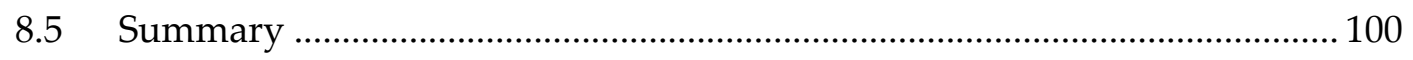

9 Validation in proposed application scenarios ......................................................... 103 


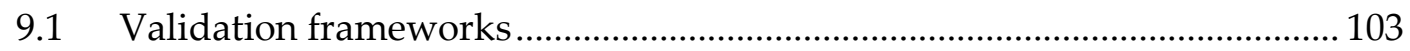

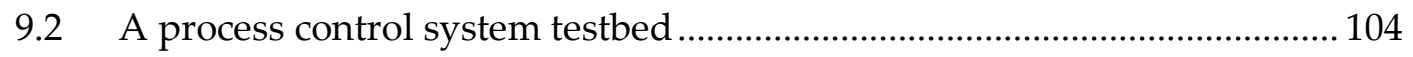

9.2.1 Results of the process control system testbed ....................................... 107

9.3 Interoperable device configuration testbed ................................................ 110

9.3.1 Results of the interoperable configuration testbeds .............................. 112

9.4 Optimizing communication efficiency testbed .............................................. 116

9.4.1 Results of applying the proposed algorithm ......................................... 118

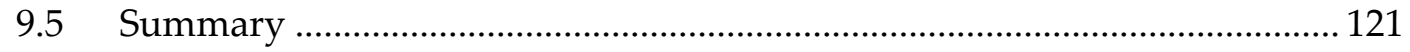

10 Conclusions and Future Works....................................................................... 123

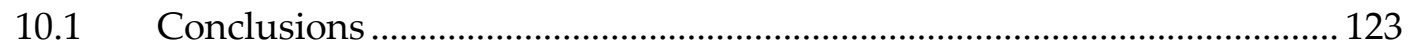

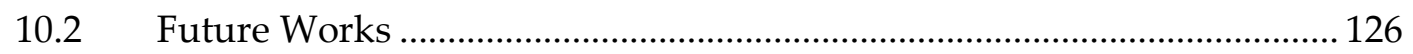

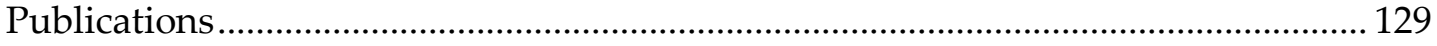

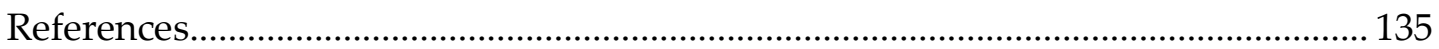




\section{List of figures}

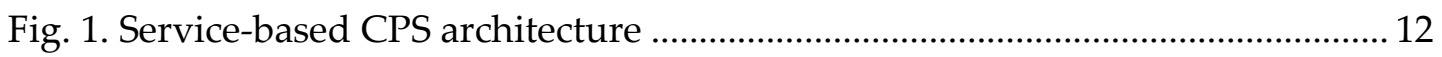

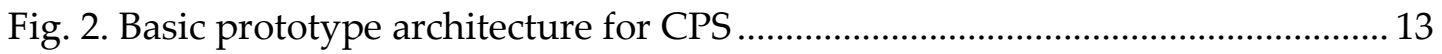

Fig. 3. EuroCPS project architecture …........................................................................ 13

Fig. 4. IoT@Work project architecture ........................................................................... 14

Fig. 5. CPS architecture model from Wan et al. ......................................................... 14

Fig. 6. Networked CPS architecture from Lai et al..................................................... 15

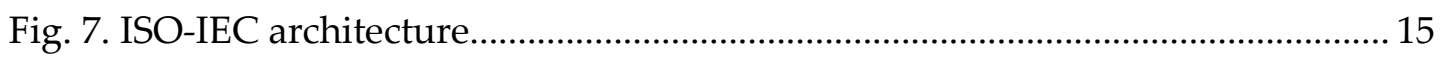

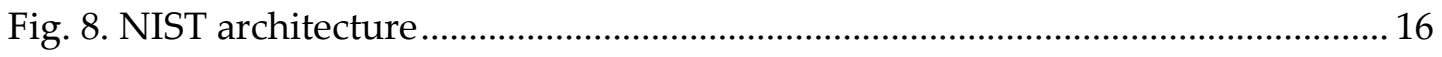

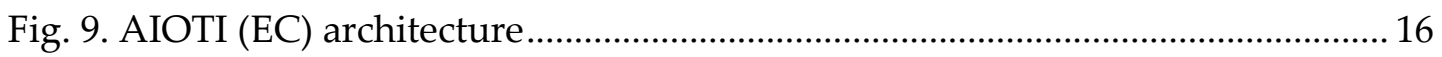

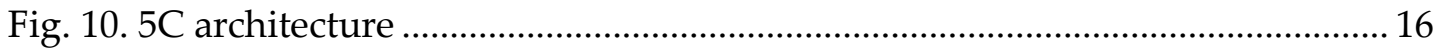

Fig. 11. Components of the service architecture .......................................................... 28

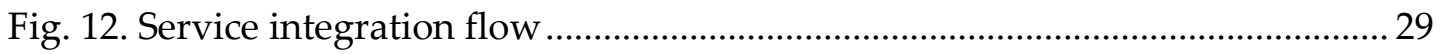

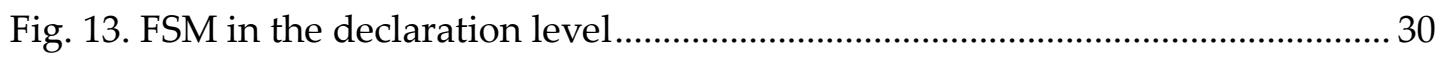

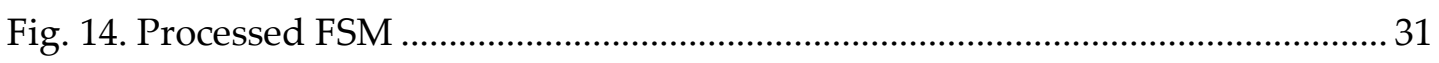

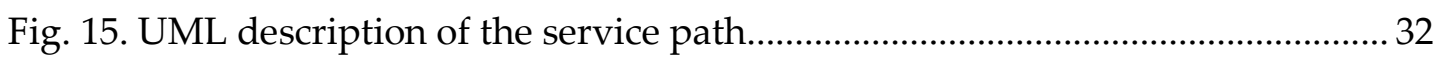

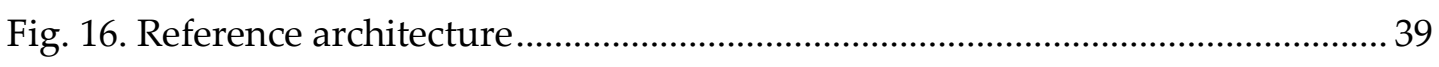

Fig. 17. Detailed reference architecture...................................................................... 40

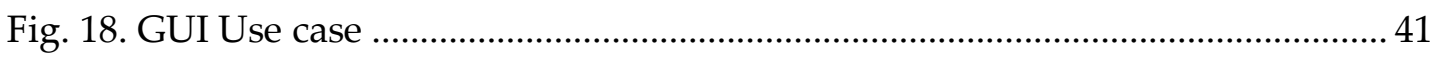

Fig. 19. Management environment distribution .......................................................... 42

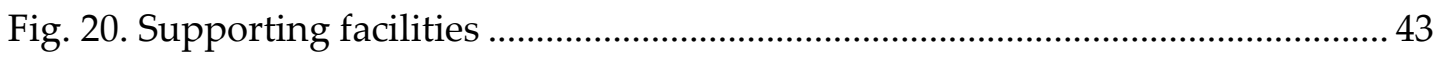

Fig. 21. Coordination Paths and multi-site data normalization .................................. 44

Fig. 22. Service-oriented Smart Environment scheme ................................................. 51

Fig. 23. Reference architecture for a fast-self configuration solution........................... 53

Fig. 24. Functional architecture of the fast-self configuration solution ....................... 56

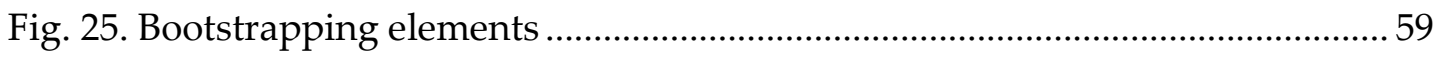

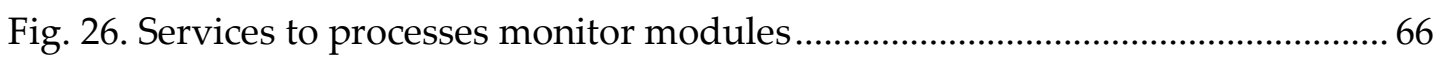

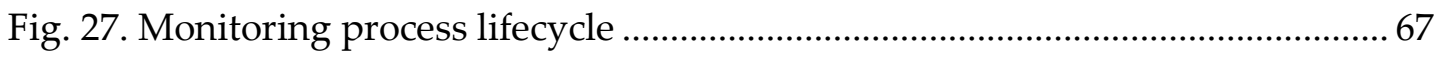

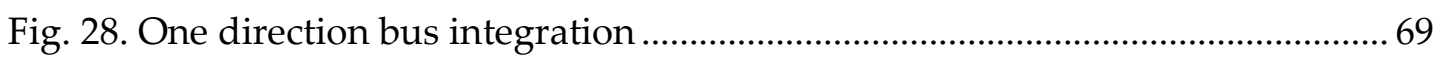

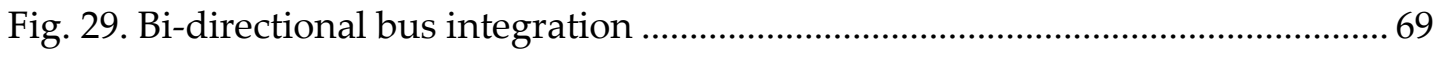

Fig. 30. Monitoring schema in a multi-site CPS .......................................................... 71

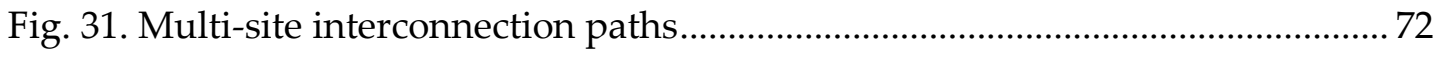

Fig. 32. Multi-site interconnection paths with central hub........................................ 73

Fig. 33. Monitoring modules of the supported architecture ....................................... 74

Fig. 34. Architecture for a communication endpoint-server link in CPS ................... 79

Fig. 35. Lifecyle of endpoints according different behavior models............................ 84

Fig. 36. Mean communication efficiency for predefined pattern servers ..................... 96

Fig. 37. Mean communication efficiency for Bernoulli pattern servers........................ 96

Fig. 38. Mean communication efficiency for Poisson pattern servers.......................... 97

Fig. 39. Mean communication efficiency for different compression algorithms........ 97 


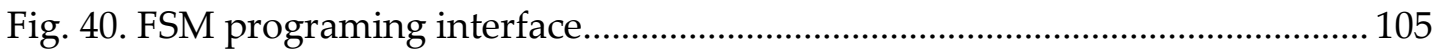

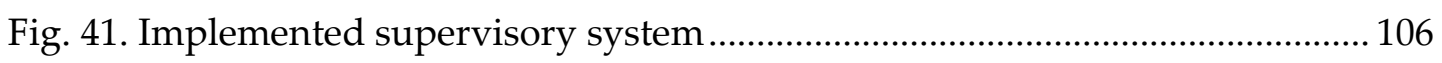

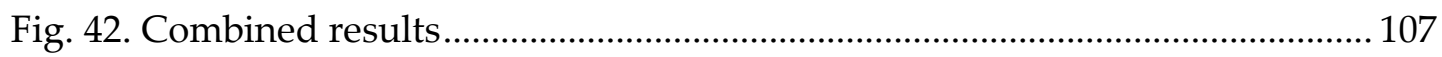

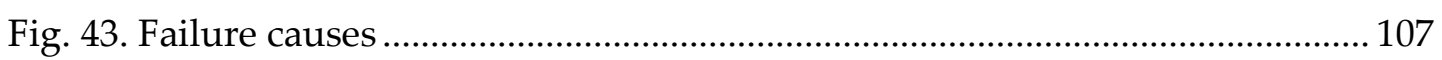

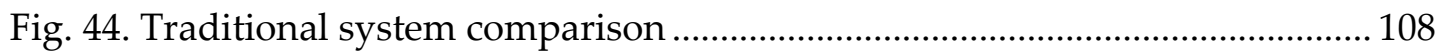

Fig. 45. Response time incrementing events/minute rate .......................................... 108

Fig. 46. Successful events percentage incrementing events/minute rate.................... 109

Fig. 47. Response time incrementing number of processes........................................ 109

Fig. 48. Successful events percentage incrementing number of processes ............... 110

Fig. 49. Normalized initial convergence time (s) - first phase .................................... 112

Fig. 50. Normalized initial convergence time (s) - second phase............................... 113

Fig. 51. Convergence time - second experiment.......................................................... 114

Fig. 52. Normalized resource consumption - third experiment................................ 115

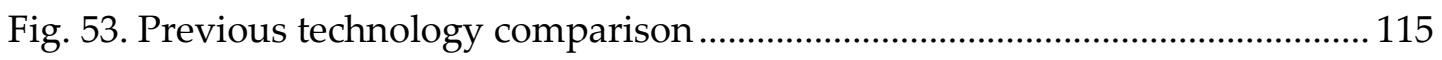

Fig. 54. Real implementation of information endpoints for CPS............................... 117

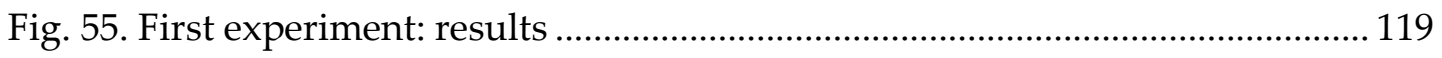

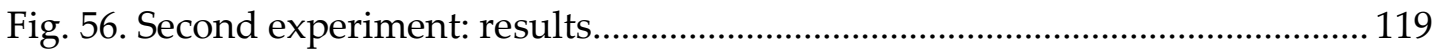

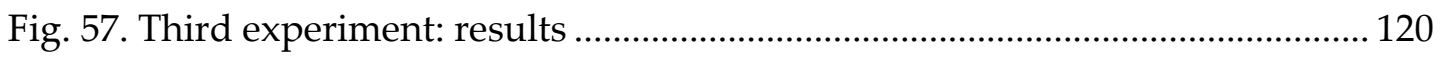




\section{Lists of tables, algorithms}

\section{Tables}

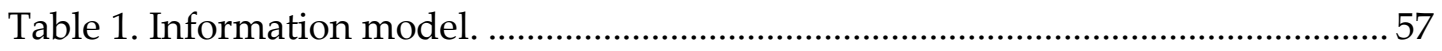

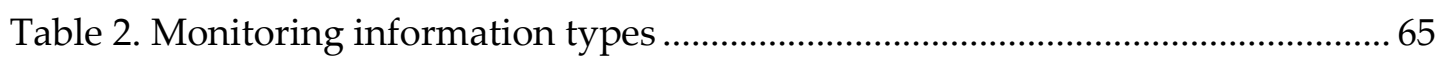

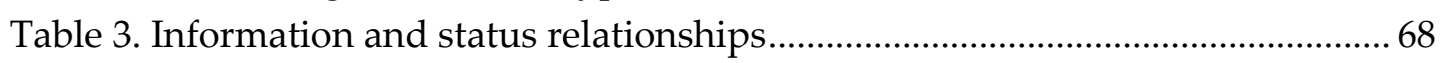

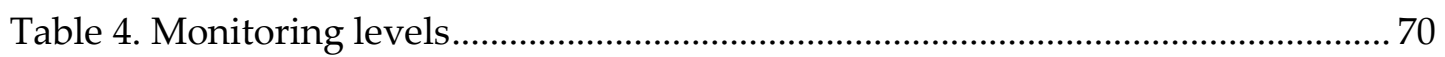

Table 5. Comparison between Pub/Sub and Req/Res patterns.................................... 74

Table 6. Total number of generated blocks. Random variables..................................... 85

Table 7. Total number of lost blocks. Random variables............................................... 87

Table 8. Link management costs for different endpoint's models ............................... 90

Table 9. Query process cost for different end point's models ...................................... 91

Table 10. Query process cost for different endpoint's models ................................... 92

Table 11. Information consumption cost. Random variables...................................... 93

Table 12. Application limits for each compression algorithm .................................... 98

Table 13. Values acquired in each scenario - Second phase. .................................... 111

Table 14. Configuration parameters for the experimental validation ........................ 118

Table 15. Fourth experiment: results ............................................................................ 121

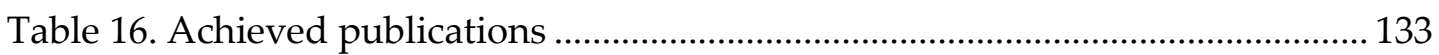

\section{Algorithms}

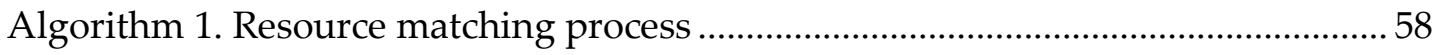

Algorithm 2. Dynamic communication efficiency optimization ................................. 99 


\section{Acronyms}

\begin{tabular}{|c|c|}
\hline AIOTI & The Alliance for the Internet of Things Innovation \\
\hline API & Application Programming Interface \\
\hline BLE & Bluetooth Low Energy \\
\hline BPEL & Business Process Execution Language \\
\hline BPNM & Business Process Model and Notation \\
\hline CPI & Cyber-Physical Internet \\
\hline CPS & Cyber-Physical Systems \\
\hline CPU & Central Processing Unit \\
\hline DB & Database \\
\hline DNS & Domain Name System \\
\hline FSM & Finite State Machine \\
\hline GUI & Graphical User Interface \\
\hline HTML & HyperText Markup Language \\
\hline IoT & Internet of Things \\
\hline ISO & International Organization for Standardization \\
\hline JCR & Journal Citation Report \\
\hline JSON & JavaScript Object Notation \\
\hline KPI & Key Performance Indicator \\
\hline LAN & Local Area Network \\
\hline LED & Light-Emitting Diode \\
\hline LXC & Linux Container \\
\hline MMTC & Massive Machine Type Communications \\
\hline MQTT & Message Queuing Telemetry Transport \\
\hline NFC & Near Field Communication \\
\hline NIST & National Institute of Standards and Technology \\
\hline OLE & Object Linking and Embedding \\
\hline OPC & OLE for Process Control \\
\hline PLC & Programmable Logic Controller \\
\hline $\mathrm{P} / \mathrm{S}$ & Publish and Subscribe \\
\hline QoS & Quality of Service \\
\hline RAM & Random Access Memory \\
\hline RFID & Radio Frequency Identification \\
\hline RLE & Run Length Encoding \\
\hline SCADA & Supervisory Control and Data Acquisition \\
\hline SDN & Software Defined Networks \\
\hline SE & Service Environment \\
\hline W3C & World Wide Web Consortium \\
\hline WiFi & Wireless Fidelity \\
\hline WSN & Wireless Sensor Network \\
\hline XML & eXtensible Markup Language \\
\hline XASM & Extensible Abstract State Machines \\
\hline YAML & Yet Another Markup Language \\
\hline
\end{tabular}




\section{Introduction}

The emerging term "Cyber Physical Systems" (CPS) refers to the integration of computational and physical capabilities using ad hoc sensor and actuator networks, which typically are seamlessly integrated into daily living objects. However, in recent years (since 2014, approximately), works about CPS also consider other devices such as microcontrollers, legacy systems or even humans, which greatly extend the system's capabilities. Based on these improved CPS, new applications, architectures and functionalities have been reported. Moreover, this new paradigm has been also employed to develop solutions in traditional fields such as the ad hoc and wireless sensor networks, Internet of Things (IoT) or pervasive computing.

In this context, one of the most promising proposals is the so-called service-oriented CPS. In this kind of CPS, the physical infrastructure offers a collection of services by means of some software elements such as service composition engines. These services may be used to perform a certain fixed application deployed by technological experts, or might be included in a prosumer environment where non-expert users can design, build, deploy and remove their own services in a dynamic way.

The actual era of technologic information is considered as the next evolution of data gathering and data processing. Almost every aspect of our lives has something related to a data driven system that collects and process the information in a paradigm called "big data". Those systems are often composed of several devices working together in a seamless operation, and managed to provide relevant results even if the devices are not present in the same location of the manager entity. This trend has been accused by the growth of the "cloud" integrations, as they rely the majority of the process in server centers located far away of the final devices and certainly, often those distances can produce unwanted results in the operations.

Considering this global picture of the CPS situation, the final user of a CPS wants a precise and reliable process lifecycle, which ensure a seamless operation in all its levels. Mostly, the need for an interoperable system in all its inner level is required to work seamlessly in every plane and in order to do this, not only the information exchange requires an agreement, but a broader definition of interoperability is needed taking into 
account organizational and political levels that the impact the system performance. From the integrated cooperation at the module level to the final communication with the nowadays, mostly battery powered devices at the end of the chain, continuous operation with the minimal user maintenance is required.

At this point, it is clear that a feasible interaction between all the modules can accomplish the goal of an improved and optimized CPS. A solution which resembles an integrated complex structure with ease of use and automatic procedurals that helps the configuration phase of its inner systems and that, offers a more streamline and dynamic aspects in its operation.

In this dissertation, I study the benefits of several factors that when included in a system, overcome the requirements of a redesigned CPS approach. The ability to detect new elements in its associated elements and how these new capabilities are offered to the system and being able to develop new services with them are the basis of the architecture presented. Furthermore, these elements are defined in an architecture proposal to provide methods to improve their working principles in several aspects, such as energy saving procedures and monitoring. The work is completed with the proposal of verification mechanisms and some considerations are given to the final devices on the basis of acquiring knowledge. A validation scenario is also described and the results presented along with future work trends that have been identified in this work.

\subsection{Document structure}

This dissertation is structured as follows:

Section 2, Motivation, goals and objectives, describes the motivation of the thesis, the goals to achieve and the particular objectives to achieve the goals.

Section 3, State of the Art, analyzes related work regarding the contributions in this work, and how these proposals fulfill the objectives stated in Section 2.

Section 4, Service provision in unattended systems, requirements and methods defines the service provision method proposed in order to incorporate an automatic service generation into the so-called unattended systems.

Section 5, Model and reference architecture for the supporting elements, identifies the required modules to fulfill the service integration chain and presents the supporting architecture that enables the integrated facilities to overcome the requirements identified in Section 4.

Section 6, Methods and functions to auto-provision deployments, proposes a solution to provide a way to initialize and configure a new device into the system, by applying a bootstrapping model and implementing a device integration procedure from the bottom to top layers. 
Section 7, Monitor and execution validation model in an interoperable multi-site CPS, presents methods and procedures to monitor and to assess the executions of processes in a system composed of several sites.

Section 8, Optimizing communication efficiency in constrained endpoint devices, introduces several considerations to the final devices of our system, and proposes methodologies to improve battery utilization using an algorithm that includes different wake-up mechanisms and wireless transmission optimizations.

Section 9, Validation in proposed application scenarios, describes different scenarios in which the solutions presented in previous chapters are validated and compared. A CPS environment is deployed where the proposed solutions are implemented and several scenarios tested where the measurements and validations are verified.

Finally, Section 10, Conclusions and Future Works, summarizes the achievements of the contributions of this thesis work and describes current research lines continuing the work of this dissertation. 


\section{Motivation, goals and objectives}

In this section, I describe the motivation of the thesis and the goals to achieve. These general goals are detailed into particular objectives, which are identified in this section, and accomplished by the contributions described in this thesis work.

\subsection{Motivation}

This thesis work is motivated by the need of exploring current CPS deployment framework, processes and mechanisms for the provision of service-oriented smart environments, service distribution and service execution model for CPS environments. This allows final users to use offered services of a system based on the devices currently present, to build a high layer application that every business model require.

In this work, I provide a methodological approach that takes into account expert, administration and final user roles to define a CPS environment implementation with improved functions to obtain enhanced operations. This approach is focused on providing the necessary steps to the final user of the CPS to implement functions and improvements in the system and allow them to optimize several key points in the architecture.

To the best of my knowledge, a CPS in which several locations, physical or logical, are integrated (for now on, let's called it a multi-site CPS implementation), lacks of a standardized component integration or architectural guidelines to follow in order to obtain an integrated system with optimal characteristics. The design of a CPS that unveils the inherit influence of every connected device in an unattended manner is dedicated almost exclusively to the architectural implementation of the main CPS components. In this design is where the performance of the system can be optimized, and thus, the importance of an adequate component selection has to be carefully thought.

Incorporating a standardized distribution method of CPS components and integrating methods and functions to the whole environment is the motivation to propose several improvements between the architectural layer and the device layer, 
where the optimizations can vary as long as the final technology used evolves. In this work, a wide variety of technologies is considered, in an attempt to verify the proposals in as many options as possible.

As devices can be present in several locations, and all belonging to the same CPS environment, there is a need to preserve and to rely all the information about the capabilities and process execution status to the manager modules, as long as keeping the veracity and validity of the data processed. This is important in order to achieve different quality indicators and to preserve a correct operation in an unattended system.

\subsection{Goals and Objectives}

The goal of this thesis is to design and validate a service provision environment for CyberPhysical Systems (CPS) with mobile devices that improve the functionalities and interoperability capabilities of the service environment. Based on a service environment for CPS, a model of a device and services adapted to specific conditions (energy, availability, scope ...) will be provided.

To do this, definition of protocols and standardized services adapted to the provision of services to the CPS environment, including mechanisms for an unattended system: solutions, algorithms and protocols that reduce the need for human intervention, will be proposed. Once it has been defined and, to validate the results of the proposals, a proof of concept will be implemented in a real hardware system.

The environment proposed in this thesis is intended to allow heterogeneous devices in a common framework and in a multi-site environment to be discovered and incorporated to the service provision system in an unattended way, considering the possibility of off-site locations and different connection technologies. Therefore, the following specific objectives are identified:

Objective \#1: to identify the requirements that a cyber-physical framework needs in order to achieve multi-site interoperability and rapport capabilities.

Objective \#2: to define a model of a cyber-physical entity focused on improving the integration, interoperability and monitor the capacity of a system, considering the different relationships that could exists between components.

Objective \#3: to design an architecture that allows the multi-site CPS inter communication to provide an integrated and interoperable infrastructure, according to the needs defined in Objective $\# \mathbf{1}$ and $\# \mathbf{2}$.

Objective \#4: to define a service model that covers the different entities of a cyberphysical service provision chain and complies with the unattended basis of the main goal.

Objective \#5: to define the service life cycle associated with a device in order to discern the functions that the global framework must offer to the provisioned entities. 
Different layers of relevant behaviors will be identified, as several points of view need to be covered.

Objective \#6: to propose a method of auto-recognition of features and capabilities based on bootstrapping methods in the core framework. A device must be configured in an unattended manner and then incorporated to the general system with its capabilities converted into high layer services.

Objective \#7: to define temporal constraints and rules for the synchronization methods to work in a seamless way and comply with the Quality of Service stablished in the system. A definition of a coordination model and its usage is also needed to obtain the framework completion.

Objective \#8: to provide tools that allow the monitoring of the devices to verify the correct operation of the system. A scalable system is required to operate the management framework as simulated devices could be added to test it.

Objective \#9: to provide a unified and heterogeneous control interface for the management of cyber-physical systems by implementing applications, prototypes and proofs of concept able to validate the various contributions. 


\section{State of the art}

This section analyzes the work related to the proposed contributions, while linking the most important features of these technologies, projects, models or algorithms, with the objectives proposed in Section 2.

The study of the current state of the art focuses on several aspects, as they are needed to understand the relation between the classic CPS approach and the objectives that this $\mathrm{PhD}$ Thesis pursues. Aspects like the introduction to the CPS implementations that nowadays integrate the physical world with processes defined in computational machines are important. Knowing the objectives that the CPS started to fulfill in its conception and how they evolve will allow focusing on the next iteration, and providing a substantial step to overcome the complications that have emerged.

\subsection{CPS implementations overview}

Current CPS implementations require a design that allows the integration and the interoperability of various elements in different conditions. In a traditional CPS, components are often seen as independent functional boxed that need to be served with their specific requirements and therefore, not in a seamless operation.

The interoperable concept is often understood as the definition of hard schemas and fixed implementations that several components share. A more natural and broader concept of this term resembles the need of the social, political and organizational factors in order to obtain a coherent service environment that allows improving the system performance.

Traditional CPS implementations often try to relate computational and physical processes (Borja Bordel, Alcarria, Robles, \& Martín, 2017; E. Lee, 2006). In the traditional implementation of the CPS concept, computational processes are state machine definitions, where the state transition depends on a physical process (Derler, Lee, Tripakis, \& Törngren, 2013). With this approach, the behavior of the system is defined and fixed in a low level, in order to define, in a precise manner, the possible actions that each device is capable to perform. This results in a very closed system, where only a 
technological expert can manipulate and configure it. In order to extend the CPS concept to a broader use, an extension of the physical process to consider any other trigger event has been also proposed (Bordel Sánchez, Alcarria, Martín, \& Robles, 2015, p. 4). With this base, even more general CPS concepts, known as Elastic Systems (Moldovan, Copil, \& Dustdar, 2018) offer the inclusion of social entities (humans) as part of the entities present in the system. Even with this kind of flexibility, technological experts are still needed to perform configuration processes, as the system does not offer an adaptable mechanism to include the common people. At least, not without a deep understanding of the technology.

Proposals that include a mechanism to dynamically define CPS processes often requires the inclusion of the "task" concept (Rajkumar, Lee, Sha, \& Stankovic, 2010). The domain expert is also the entity in charge of extracting the task information from the state machine that identifies the CPS status. By relating the inputs and the outputs of these tasks, the definition of the process can be extracted in a global and extensible way. In this point, several levels of abstraction can be identified, as a task can be seen from different perspectives. In a first form, a human readable format is generated, and a need to transform it in a machine level format is required. Some proposals (Sztipanovits et al., 2012) define a scheme where transformation modules are placed in order to obtain the low level service description.

Regarding the transformation process, a current trend identified in the literature is to perform it by using transformation rules. Those allow the software agents (Czarnecki \& Helsen, 2003) to apply the predefined translation between human and machine languages with a compiler-like methodology (Appel, Frischbier, Freudenreich, \& Buchmann, 2013). With this proposal, the advanced techniques developed for compilers, can be also used in the CPS workflow, such as predictive compilation (Childers, Davidson, \& Soffa, 2003). Several additions have been also proved the use of the semantic approaches (Tamarit, Mariño, Vigueras, \& Carro, 2017) into the real deployments, allowing specific solutions to be embedded in general microcontrollers (Bonzini \& Pozzi, 2006).

Although there has been a significant progress, those previous proposals lack several functionalities that decrease the usefulness of the service process integration. Two relevant facts are identified.

Firstly, the needed metadata for a proper service description cannot be incorporated into the system in an easy manner. Next, the metadata language used for service description is not mature enough. Authors proposed in this regard, several extensions to existing languages in order to make suitable the description requirements into the well-known solutions such as BPEL (Appel et al., 2013; Tranquillini et al., 2012) or BPMN (Czarnecki \& Helsen, 2003; Sungur, Spiess, Oertel, \& Kopp, 2013). However, the problem persists since the engines used to perform the executions are not adapted in the same way in these proposals. 
Following the language definition, the specification of the metadata is another key issue in the transformation process, specifically the temporization constraints that every CPS must support. In this context, the previous proposals acknowledge this requisite by using complex notation, as existing languages do not support it natively. Other proposals (Kim, Lakshmanan, \& Rajkumar, 2012; Zhang, Szwaykowska, Wolf, \& Mooney, 2008) incorporate several time schedulers for allowing timing control, but the real implementation is not tested.

To overcome the difficulties found in translating the task, CPS researches have been trying to adapt a WSN (Wireless Sensor Network) concept into the task declaration process. They propose to describe the tasks as a service sequence instead of a collection of actions. Those tasks need to be invoked in order to continue with the lifecycle of the service (Kyusakov, Eliasson, Delsing, Deventer, \& Gustafsson, 2013; K. Lin \& Panahi, 2010). The invocation has to be coordinated, and to provide a synchronization constraint, a central execution or orchestrator is included into the mix (Seiger, Huber, \& Schlegel, 2018). The result is a collection of proposals that using the central orchestrator, propose the use of predefined algorithms that adapt the evolution of the system depending of the physical state of the external variables (Marrella \& Mecella, 2017). With this approach, the timing constraint is easily manageable, as the central entity can obtain the current state of each module and delay the execution if needed, but the need of updated low-level information still remains.

Even more complex, can be the idea to incorporate other elements or services hosted in the internet into the system, in addition to the local devices. The on-site locations can be provided by a cloud provider or a distant headquarter of the company (J. Wan, Zhang, Zhao, Yang, \& Lloret, 2014). The service workflow in those situations must be defined, and the particularities identified to provide a concurrent deployment.

In those cases, where several locations can be added to the same CPS, the use of a central coordination entity becomes more important, and delegation algorithms have to be deployed to allow an inter-location service execution. A simple implementation of the algorithm (Clauß \& Schulte, 2014), the central entity is in charge to activate the edge nodes if a task is being executed in its location. This solution considers that each task is treated as an independent job, as the central coordinator schedules the task until its completion. This proposal, although it is proved to work, presents a very strict task delegation process, and does not adapt to changes in the platform. A proposal extending the previous technique tries to include software agent instead of the edge nodes. Those agents sit as a middleware offering public end points to be triggered by the central entity, and providing the cost of the utilization as feedback (Mueller, 2015). The utilization of these methods presents themselves several problems like standardization of the methods and the object serialization required to work.

In this PhD Thesis, an improved architecture for CPS is proposed. Focusing on the "multi-site" environment concept, a support for on-site locations is provided and several contributions made in order to ease the configuration phase of the system. As several 
works have been undertaken to improve the service availability in a broader CPS environment, I propose the addition of an adaptation process and a configuration phase to deploy a service-oriented CPS.

\subsection{Service-oriented CPS frameworks}

In this section, I analyze several CPS architectures that are found in different drafts by standardization organizations or in the literature.

Ten relevant CPS reference architectures have been identified and described. A figure is added to each architecture to understand the differences between them:

- ARCH\#1. Service-based CPS architecture (La \& Kim, 2010). This architecture provides the highest orientation to traditional systems focused on process control. It includes the physical layer, a control module that manages the system and, in this case, a service framework is included as a novel element. This element is in charge of performing all the control tasks that the system requires. One weak characteristic can be found in its low integration level. This disadvantage needs to be improved if more advanced executions are desired, as the efficiency will be improved. Additionally, advanced functions like self-adaptation or process interoperability cannot be included if there is no independent levels or adaptors included.

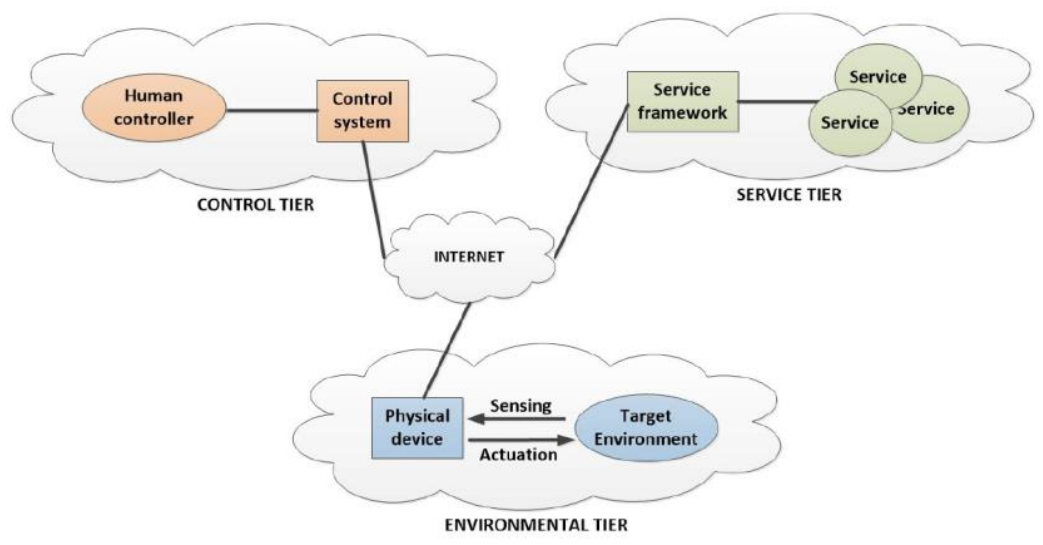

Fig. 1. Service-based CPS architecture

- ARCH\#2. In order to improve a few aspects of ARCH\#1 (Tan, Goddard, \& Pérez, 2008) proposed a basic architecture of a CPS. With a focus on an event-based architecture, they propose using publication and subscription methodologies in the processing network that allows control policies in hard and soft levels. In addition, a knowledge extraction functionality is included to gain an independent layer between the hardware and the high-level events. This approach of the low-level layers consider a virtual representation of the hardware devices in order to assign different states to the same entity that can be changed in relation with the received events. One disadvantage of this architecture is the complicated control of the processes that include several devices, as there is no central management module in charge of the uniformity of the results provided by the lowest layers. In this 
context, managing the global system status in a descriptive way is a hard challenge as the information is distributed along the whole system. This complicates a realtime control and the required calculations may overload the infrastructure in the action evaluation phase.

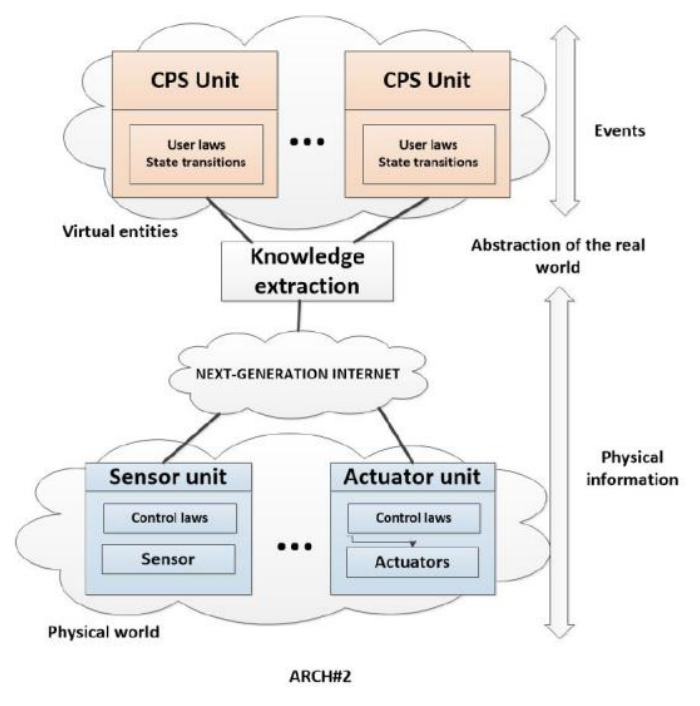

Fig. 2. Basic prototype architecture for CPS

- $\quad$ ARCH\#3. EuroCPS proposal architecture. In the European project EuroCPS, this architecture was proposed ('EuroCPS I Cyber-Physical Systems', n.d.). A collection of embedded devices is considered as a networked CPS integration. Because of the simplicity of the proposal, the architecture can be very flexible, as it can be adapted to represent almost any current system as a collection of interconnected entities. This, in fact, leverages the specific needs of any application, as the architecture does not make clear how to integrate any additional functionality into the system.

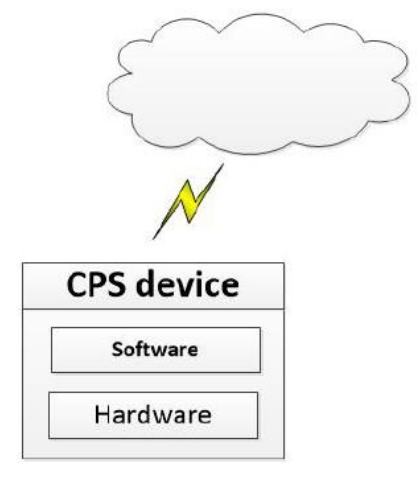

Fig. 3. EuroCPS project architecture

- ARCH\#4. IoT@Work proposed architecture. IoT@Work project (Houyou, 2012) proposed this layer approach. Originally focused on the IoT paradigm, it was not proposed to be a CPS architecture itself. However, there are some literature researches that have been used this architecture in CPS applications. The architecture solves some problems related to the layer interoperability present in the service-based environments. As it focuses on automation applications, the deployments of the Industry 4.0 paradigm benefit of the improved layer 
differentiation of the proposal. This proposal lacks however, of a solution for the geographic distribution of production locations, where it should be more specific on the implementation methods that it proposes.

\begin{tabular}{|c|}
\hline AUTOMATION APPLICATIONS \\
\hline APPLICATION LEVEL AND \\
MIDDLEWARE SERVICES \\
\hline $\begin{array}{c}\text { DEVICE RESOURCE CREATION AND } \\
\text { MANAGEMENT SERVICES }\end{array}$ \\
\hline $\begin{array}{c}\text { DEVICE AND NETWORK EMBEEDED } \\
\text { SERVICES }\end{array}$ \\
\hline $\begin{array}{c}\text { FIELD/CONTROL INFRASTRUCTURE } \\
\text { AND NETWORK }\end{array}$ \\
\hline
\end{tabular}

Fig. 4. IoT@Work project architecture

- $\quad$ ARCH\#5. CPS architecture model (Jiafu Wan, Chen, Xia, Li, \& Zhou, 2013). This proposal focuses almost exclusively on the hardware layer and its components. It differentiates three subsystems that contribute to the global infrastructure: a computing platform, storage platform and a wireless sensor network (WSN). This infrastructure takes the traditional process control methodologies from the $\mathrm{ARCH \# 1.} \mathrm{The} \mathrm{proposal} \mathrm{is} \mathrm{ambiguous} \mathrm{in} \mathrm{the} \mathrm{integration} \mathrm{layer,} \mathrm{where} \mathrm{the} \mathrm{connectors}$ with other system layer lack of a formal description and the interoperable characteristics with other technologies are not considered. In this proposal, a valuable component is included: an intelligent decision-making component including social entities (humans) is described in order to integrating all the data gathered from the hardware layer.

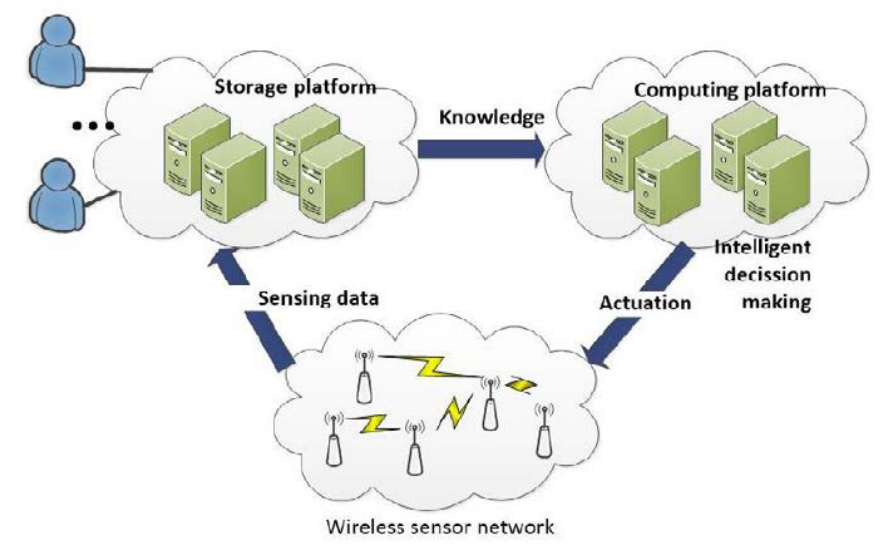

Fig. 5. CPS architecture model from Wan et al.

- $\quad$ ARCH\#6. Networked CPS architecture (Lai, Ma, Chang, Chao, \& Huang, 2011). This approach is very similar to $\mathrm{ARCH} \# 2$ in applying the same traditional concepts found in every distributed system. Additionally to the ARCH\#2 proposal, it adds an adaptation layer to interact directly with virtual entities, and even with the real world devices. This characteristic is a valuable inclusion that enhances the interoperability at the sensor layer. 


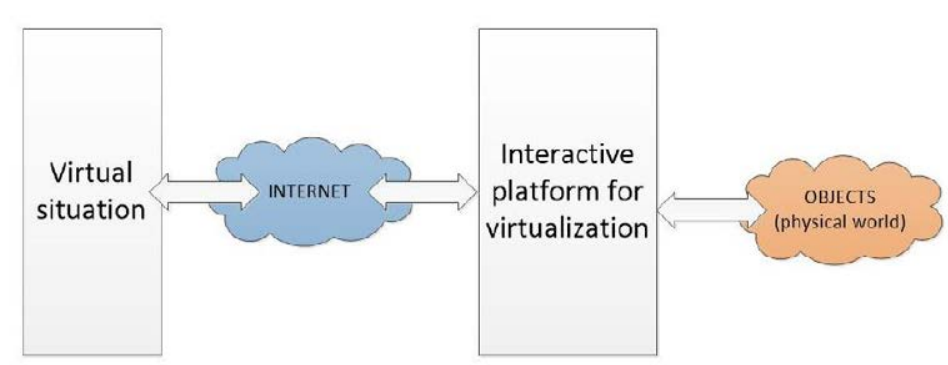

Fig. 6. Networked CPS architecture from Lai et al.

- ARCH\#7. ISO-IEC architecture. This architecture is proposed by the ISO organization in one of their research studies related to the IoT paradigm ('Study Report on IoT Reference Architectures/Frameworks - PDF', n.d.). In a first approach, it was only applied in IoT environments, but later a CPS implementation was proposed. The architecture proposal is conceptual, but contrary to ARCH\#4, it defines the concepts and interfaces better. Service-based environments are mixed with virtualization methodologies in order to obtain an adaptable system. Although, it does not include any knowledge extraction module in its layer architecture to separate the information from the low-level and the high-level layers.

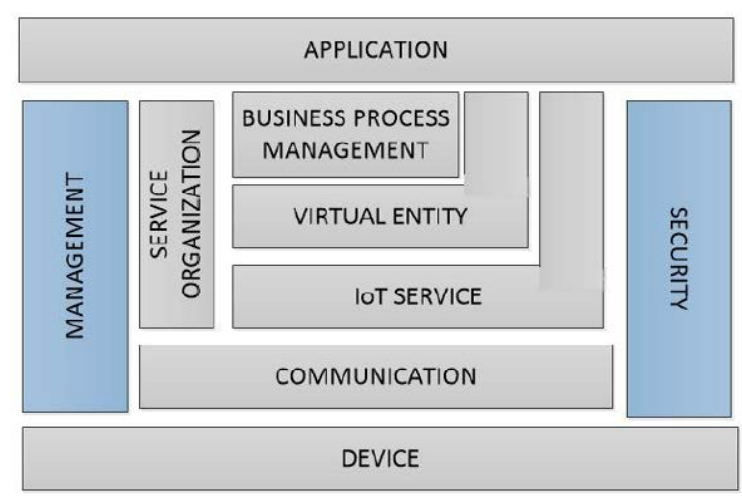

Fig. 7. ISO-IEC architecture

- $\quad$ ARCH\#8. NIST architecture. The National Institute of Standards and Technology proposed this most recent definition in 2014. It is a CPS-focused architecture that has been used in industrial environments (Thompson, 2014). All the Industry 4.0 requisites are considered in this proposal even if the cited paradigm is more recent. Several layers are identified, and a clear definition of the interfaces are stated in the proposal. From the business control layer to the low-level hardware devices, the interoperability constraints are considered, only lacking process control methodologies in order to obtain a complete service chain architecture. 


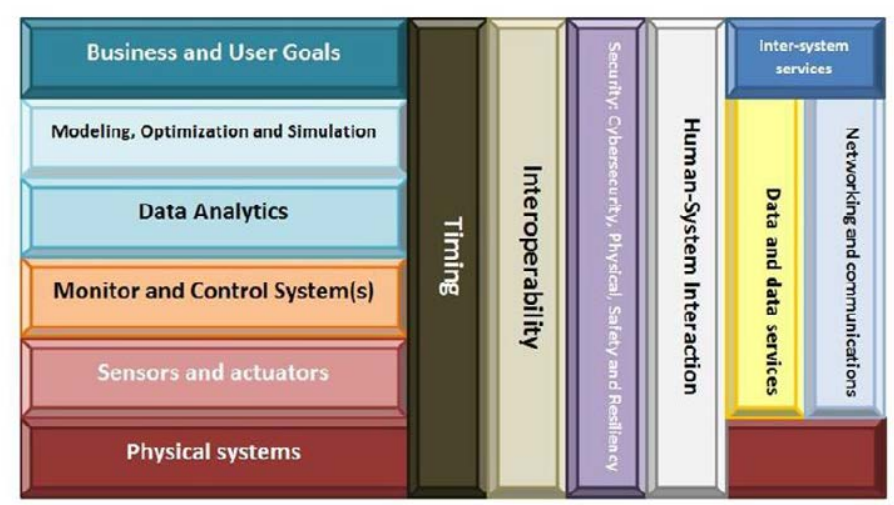

Fig. 8. NIST architecture

- ARCH\#9. AIOTI (EC) architecture. Recently, the European Alliance for Internet of Things Innovation (AIOTI) has provided an architecture focused on industrial scenarios. Taking as a base the $\mathrm{ARCH} \#$, this proposal is much more traditional that the proposed by NIST. The same traditional process control is maintained, and the low layer is changed in order to provide some improvements to the ordinary sensors.

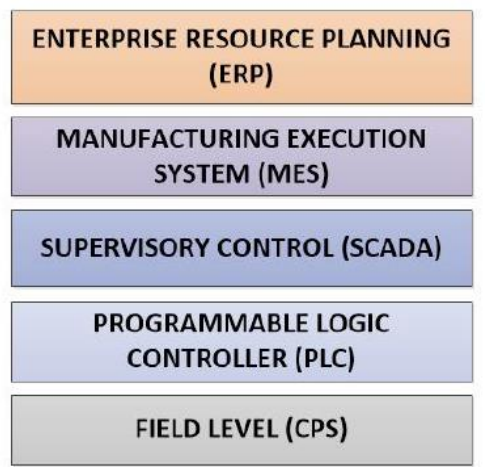

Fig. 9. AIOTI (EC) architecture

- ARCH\#10. 5C architecture. Several functions are identified in this proposed architecture. This proposal enforces the requirements that a CPS deployment must fulfill in order to solve key issues identified in the traditional information technology procedures. These functions can be used to provide a check to every CPS infrastructure but may be complicated to build a real deployment only with these definitions.

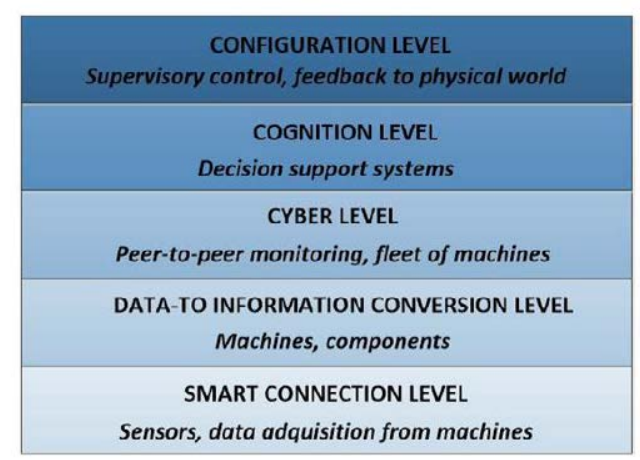

Fig. 10. 5C architecture 
In every architecture researched, there is a lack of support for automated algorithms to allow the use of intelligent environments to provide best choices and improve user satisfaction along the system, in order to improve the information offered to the superior layer, and eventually to client applications. Some works use agent-based social simulations to help creating a proper notion of the customer desires and roles.

The process of data exchange between all the architecture modules has been executed until now in a traditional and manual system. The inclusion of automated processing has opened a new window and allowed to perform complex operations. The events in which the information needs to be updated are often set by the agents involved in configuration process and often this information has to be changed manually through the whole operation. New specialized technologies supporting information are helpful in this process. Facilitating the task of updating information by reusing these infrastructures avoid a waste of time in these procedures.

In this PhD Thesis, taking into account the reviewed CPS architectures, and with a critical vision of their strengths and weaknesses, an improved version is presented by selecting certain modules present in the reviewed architectures. In Section 5.1 the proposed architecture is described with a special focus on improving the interoperability capabilities of the global system. In addition to a thorough requirement gathering and to complete the previous architectures, a scalable and multi-site supported infrastructure for CPS is then completed and validated.

\subsection{Service based CPS configuration and adaptation backgrounds}

In order to obtain a general picture of the current service-based CPS methodologies to allow an automatic and adaptable configuration of the service lifecycle, an analysis of the literature is made. Service Environments (SE) are based on two procedures that must work seamlessly to provide the configuration and the adaptation mechanisms to the CPS that are required for a successful service provision.

The configuration process in a CPS entails the installation of the system components in order to allow the inclusion of new functionalities. This procedure interacts with the new device to obtain the parameters and initial information needed to start operating. As is, this phase is located in the pre-operational phase of the operation ( $\mathrm{Hu}, \mathrm{Zhang}$, Zheng, Yang, \& Wu, 2010). In small deployments, however, an automatic process may not be needed as the number of devices to configure is low enough for a manual process, but in practice, when the number of devices grows, an automatic solution needs to be considered. In a first approach, a semi-automatic process must be at least included, to complete the first configuration phase and allow a manual completion of the process, once the information is manageable.

To provide adaptation mechanisms, other approaches should be considered. As the adaptation entails the dynamic modification of a system without intervention, the system must support to continue operating and adapting at the same time. The basic concepts in adaptation procedures comprise the addition or removal of the entity 
components of the system (Erradi, Maheshwari, \& Tosic, 2006). These changes occur at any time when the system operates, so the behavior of the system must be compensated and corrected at operation time without being interrupted. In order to accomplish those requirements, the adaptation process should be automatic and fast enough.

In this section, I try to analyze the backgrounds and the current literature related to the configuration and adaptation process in a CPS that fulfills the automatic requirement of large deployments. These proposals can be divided in two groups.

To start with a classification of the automatic configuration procedures found in recent proposals, solutions including the hardware virtualization are the most used by authors. The idea behind this concept is to create a physical model of the devices and virtualize each entity into the system. This allows extracting a list of the available capabilities and configure the devices based on a predefined map. Some of these proposals developed a middleware layer to include there the virtualized elements (Familiar, Martínez, Corredor, \& García-Rubio, 2012; Familiar, Martínez, \& López, 2012). Other works propose the inclusion of an agent-based implementation where every entity is represented into a directory (Alcarria, Robles, Morales, \& González-Miranda, 2012), and allow extending the solution to hybrid systems. With this approach, several problems are identified. First, a domain expert must be included in the first phase to extract the services of the domain knowledge and then, manual intervention is still needed to update the directory of services, or to change the information related to certain service. In addition, a second problem is that the independency is not achieved between the service abstraction layers, as the domain experts require to be in charge of the hardware level and the service level, to provide the high-level services to the application layer.

In this group, a special mention can be done to the solutions based on semantic technologies (Gouin-Vallerand, Abdulrazak, Giroux, \& Dey, 2013). They often include the semantic process only into the middle layers of the system, and it is employed to abstract the lower layer of the system and provide an independent layer in between the high-level layer. The semantic process is in charge of relating the meaning of the services and detecting a similar service in the catalogue. Because of this, similar problems than previous solutions also appear in these proposals.

A second group of proposals provide solutions that involve the exchange of large amounts of data, between all the entities present in the system. With this approach, authors expect that the state of the system, described in an in-depth form, is shared between all the entities and replicated. With this information, each entity must depict their own state. The automatic part of this procedure is then trivial, as every entity is in charge of itself; the system only has to run a mechanism to configure the interconnections at the start of the operation. Then the system operates until a new entity wants to be incorporated. As an example, some authors propose a system with a self-configuration mechanism including a distributed description file (Mönks, Trsek, Dürkop, Geneiß, \& Lohweg, 2014). Those systems do not require any adaptation mechanisms, (and it can be 
seen as an advantage), but the computational effort in generating, distributing and analyzing the state description can be impossible due the nature of some CPS devices. In addition, the independent layers are not kept, and the domain expert must know the whole system in order to interact to it.

As said previously, a configuration process may need a system reset and reinitialization when a change in the system occurs. In order to allow resuming the operation without a restart, self-adaption procedures can be added. The following description tries to extract the current state of the art in this concept.

To add adaptive configurations, authors nowadays implement algorithms in order to react to the unexpected changes of an infrastructure. A proposal (Smirnov, Kashevnik, Shilov, Makklya, \& Gusikhin, 2013), for example, tries to complement the software agents with recurrent algorithms in order to provide a negotiation between the entities and then an information exchange by the use of predefined protocols. Another approach (Dillon, Potdar, Singh, \& Talevski, 2011) adds adaptation procedures by using a scheduler in each device that communicates with the central entity in charge of the coordination task. A project called SOCRADES (A.-W. Colombo, Karnouskos, \& Mendes, 2010), a proposal of adaptation mechanism using petri nets is made, but the need of a manual process problem persists. In the last year artificial intelligence and machine learning have been used by projects like AutoPnP ('AutoPnP', n.d.), to provide the addition of new components.

The described proposals, although they provide different solutions to the problem, the complex algorithms used and the need to maintain a current state snapshot of the platform distributed in the entire infrastructure makes difficult the execution of the solution in real world deployments. The time constraints and the reduced computational power cannot produce the expected results.

To overcome the key issues found in the current literature, my proposal describes two different mechanism that allow executing the required operations and provides an accelerate configuration process. Firstly, a semi-automatic process is defined. With the help of a domain expert in the first stages of the deployment that extract the knowledge of the application and the use of semantic templates, the infrastructure is able to gather all the information needed. Then the information is converted automatically, and the independency of the levels are maintained.

\subsection{Automated service distribution at location level}

In recent literature related to system infrastructures that can integrate several colocation sites in one unified management entity, several approaches can be identified. Companies and database users are now aware of the benefits of cloud-based solutions for data provision and processing. Reliability is an increased concern over the heterogeneous options regarding cloud computing and distributed database services. 
Previous related work in the distributed queries scenarios falls in the area of implementing different methods to join the diverse database results over the cloud. Researches concerning the integrity of the distributed queries involving confidentiality of the exposed data in an untrusted computational cloud are now aware of the problem of joining multiple data sources. These companies and other authors (Vimercati et al., 2014), proposed an approach to allow users to assess the integrity and privacy of the merged results. Enabling encryption on the fly algorithms and storing partial results on the unsecure servers allow them to use MapReduce paradigm to perform the final join. Other work (Chidlovskii \& Borghoff, 1998) motivates the split of the original query and the inclusion of fake tuples into the dataset to replicate data and then, by executing a precise query and combining the result, achieve to generate a more concrete output. They combine adoption of twins and markers to guarantee integrity of data, which it has also been discussed in using a semi-join evaluation strategy.

Besides normal queries, the number of aggregated queries a server can handle in a certain interval is limited to certain amount until the server is saturated. Every query pattern has an individual schedule and different window size and frequencies. Studies (Chen, Xiao, \& Liu, 2010) address the problem by identifying the requirements of each query and scheduling them in an efficient way using algorithms to identify the need of executing a query less often as long as the interval of repetition is minor compared to others. Combining the scheduling algorithms with earliest-deadline-first methods to handle under-loaded and over-loaded situations, the authors are capable to dimension an optimum system for the specific scenario.

Following a distributional query paradigm, supposing a content delivery scenario, different contributions (Loyall et al., 2012; Robles et al., 2015) introduce a new concept for publish-subscribe networks that focus on information dissemination and the quality service control in order to maintain a good level of reliability in the system. This is especially important in dynamic environments as the asynchronous responses could not be in the expected time interval. Using Publish-Subscribe paradigm for inter-application interfaces allow us to maintain an optimum routing control and QoS.

The inclusion of database observation and monitoring are now starting to take part along with smart objects with sensor capacities and CPS services that incorporate data acquisition and generation by themselves (Crisan \& Rantzau, 2008). Observing IoT resources allows servers to monitor state changes and to notify to an interested client without the need to involve a database in the process. Notifications in this case are aware of the real-time events but lack the historical data (Klie, Fischer, \& Mueller, 2011).

Researches (Gannon, 2006) often involve asynchronous ways to perform notifications in a user-role system, like in social network, software environments use a mix of web services real-time technologies to achieve a real event-based notification for serviceoriented and monitoring purposes. The mentioned comparative study analyses the Publish-Subscribe architecture for event dissemination in distributed system. Using publish-subscribe architecture allows data events to be delivered to consumers of a 
specific topic based on subscriptions (Morales, Robles, Alcarria, \& Cedeño, 2013). In addition, systems can be configured with as many brokers as needed, providing flexibility and scalability.

\subsection{Considerations on CPS endpoint devices}

Low-power systems have been relevant in the last years due to the increased interest in energy-aware devices. Several CPS topics focus on how to propagate connectivity into devices with great mobility possibilities and often, a reduced consumption is needed in order to provide longer autonomy and avoid maintenance procedures. To minimize operating power, maximizing the total battery lifetime in a scenario where the wireless transmission is a key factor, low energy protocols, as well as procedures to reduce the transmitted data, have been considered to perform the final implementation in this paper.

Several wireless communication technologies have been developed to fulfill the requirements in the CPS paradigm. Bluetooth, ZigBee and the newest version of Bluetooth defined as Bluetooth LE (Low Energy) (BLE), are the most popular wireless standards nowadays. In (J. Lee, Dong, \& Sun, 2015) a comparison is made in terms of applications, power consumption, data rate and encryption. In addition, the advantages and disadvantages of each implementation are identified.

Wireless protocols like the new version of the Bluetooth technology are now focusing on energy consumption reduction. Combining the energy aware wireless protocols with compression algorithms in the source has been analyzed in (Ma, Hempel, Peng, \& Sharif, 2013), obtaining relevant results in reducing the time involved in the final transmission. However, this study lacks the impact analysis of the decoding process in the destination device. Optimizing connection between master and slave devices is proposed in (Nair et al., 2015) with an infrastructure using a hybrid topology to reduce power consumption in wireless sensor network. This allows the device to remain more time in sleep mode in which the power drain is reduced.

Data compression algorithms such as (Sharma, 2015) applies in wireless sensor networks to effectively reduce the quantity of the information transmitted through the air. Both lossy and lossless scenario approaches, exploiting the temporal correlation of sensor data can be found in literature (Mondal, Zaman, Masud, \& Alam, 2008; Szalapski \& Madria, 2014). One problem found in research literature emerges when the device is not a sensor, in charge of generating the information, but an actuator, in charge of representing the information received, and therefore the device has to decode and present it. Soft computing algorithms such as (Nimmagadda, Kumar, \& Lu, 2009) try to reduce the computing load in both, master and slave devices.

Algorithm studies involving data compression in wireless devices often focus on video transmission. A review (Pereira \& Pereira, 2015) of the state of the art in energy efficient techniques in resource constrained systems concludes that there is a two-group classification, energy-aware transmission systems and energy-aware (Puthenpurayil, 
Gu, \& Bhattacharyya, 2007) compression systems, which I am trying to unify in a lossless scenario. Electronic paper (E-Paper) (Rogers et al., 2001) intends to display information with minimal energy consumption, as it can be disconnected from the power source without losing the displayed information, low energy communications and computational energy-aware methods (Raghunathan, Schurgers, \& Srivastava, 2002) are a key to an effective power saving strategy.

Wireless sensor networks rely on improving battery life of sensors by minimizing the transmission of information (Kimura \& Latifi, 2005; Szalapski \& Madria, 2014) through the wireless channel. Optimizing the compression for efficient power allocation in multidimensional sensor clusters is analyzed in (Kumar \& Singh, 2015) and a mathematical model is developed to achieve a balanced between the transmission power and the compression power consumption. These parameters have to be defined in the configuration phase. This configuration process is a complex procedure in CPS, so it is usually addressed at different independent levels. Typically, two types of works may be found in the context of CPS endpoint devices (Borja Bordel, Alcarria, Sánchez de Rivera, Martín, \& Robles, 2018, p.): papers about network configuration and discussions about service configuration.

Proposals for an automatic network configuration are the most common. Basically, all these works are focused on basic interoperability, so the final objective of those algorithms is the component installation in the Smart Home in an automatic manner (AlKhawaldeh, Al-Naimi, Chen, \& Moore, 2016). To perform this installation operation some works employ configuration robots based on artificial intelligence, software defined network (SDN) technologies (M. Lee, Kim, \& Lee, 2015), new architectures with specific configuration middleware (Shon \& Park, 2010, p. 4) or Domain Name Systems (DNS) (S. Lee, Jeong, \& Park, 2016). Other works only discuss about security issues and other future challenges in relation to CPS configuration (H. Lin \& Bergmann, 2016). None of these works, nevertheless, consider system efficiency in the configuration process.

Network configuration solutions to improve system's efficiency are always focused on energy consumption. Some proposals are focused on efficient configuration solutions employing the minimum energy amount (Rust, Picard, \& Ramparany, 2016). However, in these works, the focus is not the system operation but the configuration algorithm itself; thus, after an energy efficient configuration the system may operate very inefficiently. Only some very sparse proposals address the challenge of efficient operation, although focused on low-energy consumption (Byun, Jeon, Noh, Kim, \& Park, 2012) and sensor-server communications (through, for example, flexible configuration middleware controlling the network architecture). These works are usually known as "energy-efficient self-adaptation" solutions, and sometimes are also applied to other similar technologies such as Internet-of-Things (Sen, 2016).

From another point of view, some authors address the CPS automatic configuration at service level. Typical proposals at this level are based on virtual sensor representations 
(Evensen \& Meling, 2009) which are mapped into the real deployments. Other works employ models and description languages to enable an automatic and dynamic system configuration (Cetina, Giner, Fons, \& Pelechano, 2009). Although in general these works do not consider the future efficient system operation, some authors refer to this requirement as a desired objective (Aberer, Hauswirth, \& Salehi, 2006).

In that way, as a general conclusion, automatic and dynamic configuration solutions for CPS or similar technologies (such as Smart Environments) are not focused on a future efficient system operation (Mayer, Verborgh, Kovatsch, \& Mattern, 2016). To address this challenge some specific system architectures (Ding, Song, Tong, \& Li, 2016) have been reported (using, for example, smart gateways) but they are focused (once more) on sensor networks and sensor-server communications.

In this PhD Thesis, I propose, on the contrary, a complementary configuration algorithm, which may operate together with any existing technology or architecture. Once endpoints are configured at network and service level (and then connectivity and interoperability are guaranteed), an algorithm to configure and modify, those characteristics affecting the system efficiency is periodically executed to improve, as much as possible, the resource usage in relation to the obtained value. 


\section{Service provision in unattended systems, methods and requirements}

In this section, I identify the requirements of a new service-oriented framework to provide an unattended model behavior to offer services to the final users. As one of the main objectives in this thesis is to develop a new architectural model, an understanding of all the requirements and the top elements of existing architectures to achieve the final provision system, is required to develop a framework in a real scenario. The outcome of this process will be a device discovery service to incorporate and share processes coming from a superior layer and executed into the final devices.

The basis of this contribution is that, as discussed in Section 3.1, there are no framework, or service lifecycle models that explicitly take into account all the relevant new parameters needed in the new IoT and CPS era. In order to discern the status of a final device or its capabilities, there is a lack of information on the needed mechanisms. In particular, in how to adapt existing process execution models to connect with the actual capability discovery methods, as the evaluation of the compliance between the relevant actors and even the Quality of Service is required.

In this section, I explain the use of the unattended concept (along with other terms such as task execution, capability discovery, bootstrapping, etc.) in CPS and IoT scenarios. It manifests the necessity of automated application processes and service development frameworks that assist the deployment (or update) of a system.

As a validation of this proposal, I follow a method that includes developing a system framework model to create and personalize the device characteristics and aligns it with a service execution engine. 


\subsection{Service-oriented CPS context}

One of the most promising trends in this regard is the so-called service-oriented CPS. In this kind of CPS, the physical infrastructure offers a collection of services by means of some software elements such as service composition engines. These services may be used to perform a certain fixed application and in certain cases can be deployed by technological experts, or might be included in a prosumer environment where nonexpert users can design, build, deploy and remove their own applications in a dynamic way.

Nevertheless, a great chance to improve CPS infrastructures was born with the definition of the Industry 4.0 paradigm, in 2014. This defined the basic concept of the next generation of information technologies, with a deep integration into the industrial context, their procedures and solutions. Similar innovations and improvements from evolved CPS are expected, as particular scenarios and applications would require them in a short period of time.

In this Thesis work, a service-oriented environment based on the Industry 4.0 requirements is used to build services that execute the application defined processes. These processes require that the used services have to be previously configured and linked to each other in a way that allows the realization of the task or a collection of them. As processes can be destined to any business environment or framework, tasks are defined by using various methods and technologies. In addition to business tasks, policy management or even assisted living control can be possible. Therefore, it is needed a process to support the execution of several services once, several times, even periodically or when other condition is met. Moreover, process chain must be expandable in several ways in order to support future implementations of the external services that may be required, such as virtual services provided by the cloud, external company middleware or various site-locations that are part of a same organizational structure. Except in the last case, remote services are invoked from the CPS to an external and uncontrolled site and the so-called CPI (Cyber-Physical Internet) may be used to interact with the public endpoint. The last case will be focused in depth in this $\mathrm{PhD}$ Thesis work as in Section 5, a multi-site architecture will be presented.

To define process and the execution order in the application layer (where the users using the infrastructure are placed) a configuration phase must be declared to allow abstracting the required services into some data base repository that holds the system information. This abstraction is used by several instruments that analyze and decompose the information and translate user desires into required actions (and even, executable code).

\subsection{Service assessment model}

In this PhD Thesis, an improvement over the classic method of service control is required, and therefore, a design of a new service model that improves the traditional 
service management process is essential. Thus, in this section, I propose the addition of several modules into the service control chain to allow a more granular service assessment process.

\subsubsection{Service control and provisioning}

In general, current architectures analyzed in Section 3.2 are focused in satisfying the industrial system's requirements and most of them does not support CPS process control mechanisms. This manner, state of the art presents little improvements over the traditional control systems, and a wide range of inclusions can be proposed. With the proposal presented in this section, a more granular service management is desired. In order to support the proposed service control method, a service path is needed to follow the activities execution process from the bottom layer to the top, and to do this, there are several proposals that divide the service path in different modules performing a specific task (S. Wang, Wan, Li, \& Zhang, 2016). Often, traditional literature is focused on Smart Grids (Sridhar, Hahn, \& Govindarasu, 2012). This is because of the recent CPS terminology, where the control policies and system implementation started to be implemented. Proposals only considering the control system related to CPS are infrequent.

My proposal addresses a gap in the actual research literature, by proposing a fully functional process control system and considering all the required functionality to obtain an interoperable infrastructure. This proposal will be completed in the following sections with a complete overview of the proposed architecture. In order to reach this objective, the reviewed CPS architectures in Section 3.2 and their design guidelines are taken into account as a basic approximation to the interoperable CPS concept. Consequently, a service-based solution that provide an event-driven approach is designed. The NIST reference architecture is considered, and the concepts of state and state transition will be described.

\subsubsection{Service data components}

To overcome the required service control, several components take part in the service composition process that is proposed, and the description of each module related to the phase of the implementation process as shown in Fig. 11, is stated below:

1. Physical system. It contains all the elements that the infrastructure must operate and monitor. These elements perform the operations and process defined by the system. Almost every hardware infrastructure and production system are part of this layer.

2. Transducers. Including actuators and sensors. These devices are the components of the physical system and can be part of a same device if they share the same case, as in production systems. They are in charge of activating or sending events in the system, by activating a flag into the hardware interface. These modules can 
be provisioned as input elements where the operation can be monitored to alter the current operation.

3. Connector access manager. This component refers to the data transportation layer that distributes the information gathered by the physical system to the control layer. In this phase, the message is codified for the data acquisition module to process it. OPC protocol is used in the majority of the industrial system reviewed, and although only one data interface was described, the control environment interface can be also included to interact with the hardware connectors. In this work, only the data connector is described, because the system behavior is the same in any case.

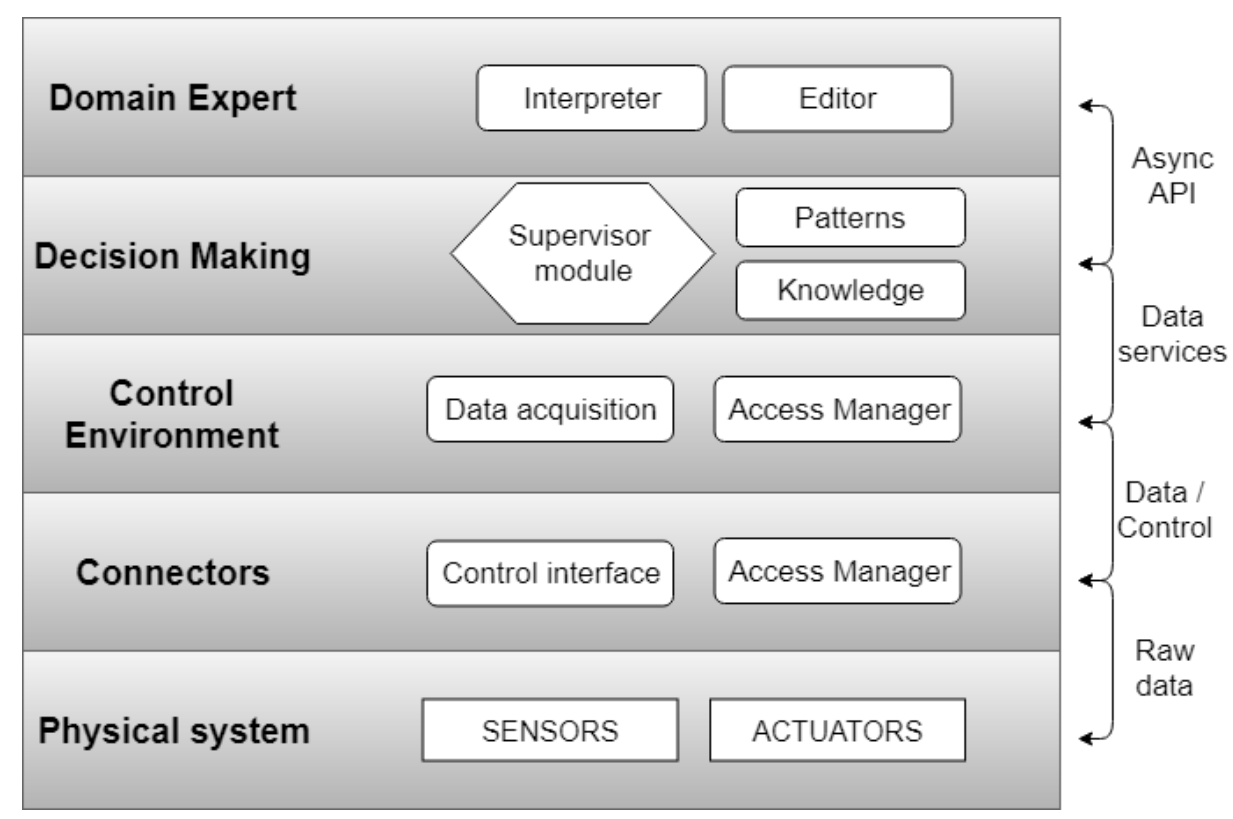

Fig. 11. Components of the service architecture

4. Control data acquisition. It is the component that acquires the data generated by the transductor layer and processes into an adequate format that the higher layers can accept. The process takes the raw data (although it can be codified by the connector middleware) and interprets it by making the appropriate changes. There are cases that those changes are implied by the access middleware, and then the control layer would be embedded in the same connector layer. A self-managed CPS would result in those situations, by integrating the lower three layers into the same module.

5. Control access manager. In order to provide a hardware control entity, at the control layer a middleware in charge of managing the access control policies is added. Any type of middleware may be chosen, thus, in this proposal an eventbased middleware is used. This allows the utilization of a publication and subscribe protocol that perfectly adapts to the lower layers.

6. Information interpretation. At this above layer, the knowledge extraction module receives the events and extracts the information. Additionally, a pattern 
module allows to identify any known pattern and rely the data into the next module. In this layer there is no need of any P/S technology as the elements can interact in a sequentially form and no unexpected events can be generated from the system. The information is passed to the next module by using an asynchronous API that allows a non-blocking process of the data, controlled by the supervisor engine.

7. Domain expert environment. The last and higher layer is in charge of receiving the events generated by the lower layers and updating the state of the execution process to the actual state. In this phase, the events may trigger some activities that produce an output, or a control algorithm can choose the next execution step in the implemented service. All of these particularities can be defined in this level, with the help of a domain expert. The control of the system is then leveraged to the activities described in the editor, and then processed by the interpreter. Managing the system is done by selecting a human understandable language that allows the domain expert to translate the application specific requirements into the system activity description.

This is the workflow proposed to integrate application services into the system, as seen in Fig. 12: in a first step, the domain expert describes the application layer model into a finite state machine, using activity descriptions and other domain specific tools. Then, the interpreter compiles the information into a machine understandable code.

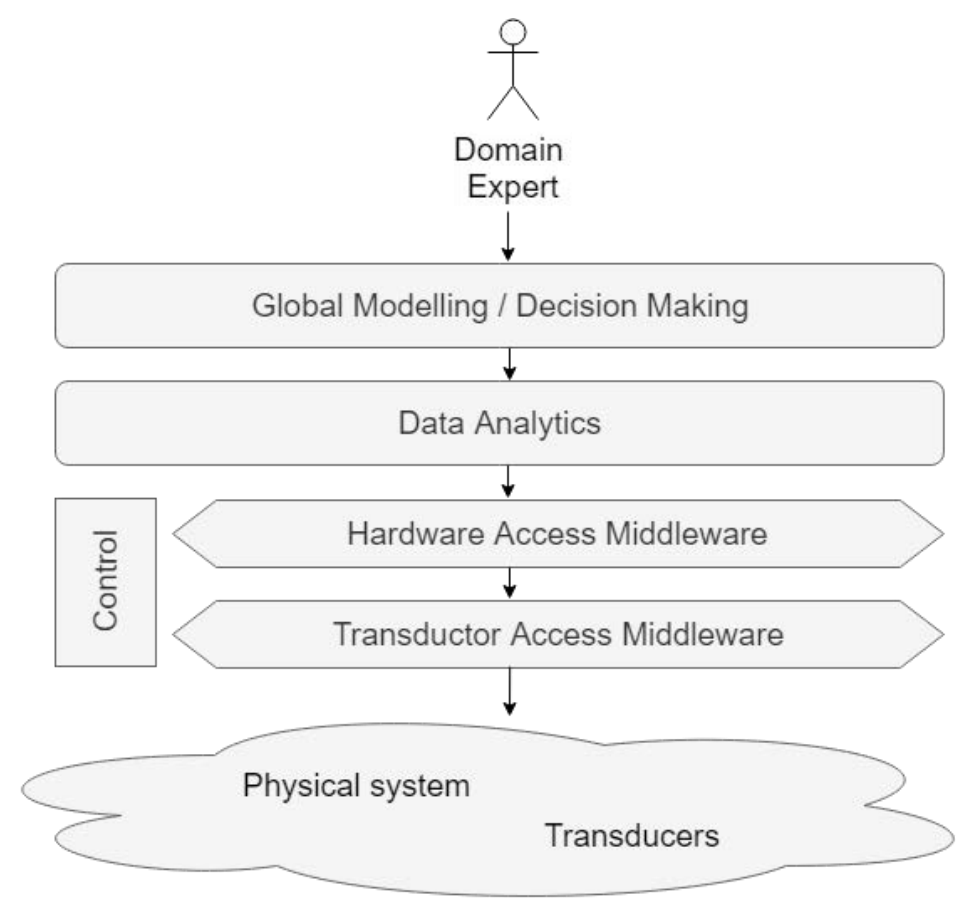

Fig. 12. Service integration flow

Once the transitional code is generated by the supervisor module, the state transition table is put into memory and the needed subscriptions are requested. Business events can be also provided, as the business layer maintains an independent state from the 
lower layers. These events coming from the upper layer are the foundation of the decision-making processes that may be included in the transition table. In order to make an update to the current state machine, the execution orders can generate activity events that in combination with the prosumer events described by the domain expert produce the output sent to the final users. This is when the users can be noticed and get feedback of the system executions.

\subsubsection{Integrating a service generating process}

As shown in Fig. 15, the service chain is described by splitting the levels corresponding to different functionalities. Although, none of them can work without its subsequent level in the chain, a modularization has been done to provide a granular description of each process.

In a first level in the service-provisioning plan, the domain expert is placed to provide the necessary data to enable the service chain infrastructure. In order to obtain the information, it is needed to define the declaration format of the service provisioning data. A finite state machine (FSM) declaration as shown in Fig. 13 is defined as the primary state of the information gathered by the domain expert. It contains the process declaration, a list of states in which the process can be found and its associated activities. In addition, the entry and exit activities are also required. This collection of atomic operations can be seen as the service invocation operations found in other service-based environments.

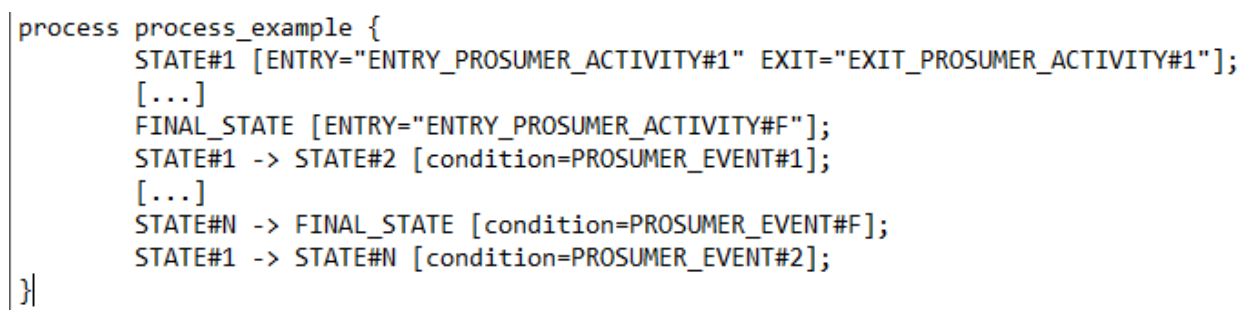

Fig. 13. FSM in the declaration level

As it was previously said, the domain expert registers and declares the FSM. By using an interpreter, the domain expert can transform the high abstraction level that is inherited from the information gathered into an executable code that can be understood by the rest of the system. In this step, the prosumer code is then transformed into a lower level of abstraction. It is important to note that the transformation process takes place in the first level of the chain, and the domain experts can monitor the process and edit the transformed data if wanted, with the help of an editor. The languages used in the transformation process can be selected, as long as the interpreter and the editor use the same. $\mathrm{XML}$ as defined in the W3C state chart or preferably executable description languages such as .NET or XASM language can be useful in this level.

This level offers the domain experts an interface to interact with the system, in a high level of abstraction to allow using a human understandable format. This upper level must be independent from any bottom layer, and thus, it can be moved to other system platform if desired. The domain experts can therefore select their language of coding in 
which the FSM can be described, as they are many available. This is where a language focused on a specific requirement of the application can be selected, or even better, select various languages for each application specific elements that fit best in order to produce descriptions that are more accurate.

The result of this level is a business description of the activities used in the services, as can be seen in Fig. 14, and being independent from the hardware level, helps the separation of the layers in a soft manner. This allows the reutilization of the premises with other hardware infrastructure.

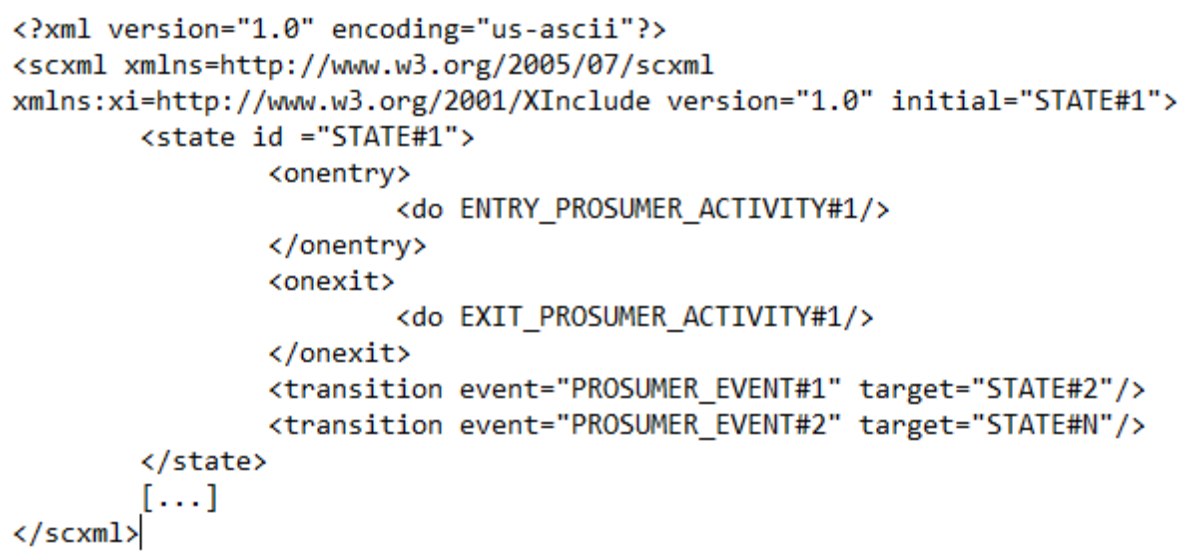

Fig. 14. Processed FSM

Once the new description is generated, the information can be transferred to the next level in the chain. In this second level, the execution environment adapted to the states identified before must be generated. For the execution environment to be ready, a proper execution engine has to be provided, which understands the business level (or decisionmaking level) description language coming from the interpreter. As an example, a C++ execution engine is needed if .NET technologies have been used in the domain expert level.

In a service-based environment, services have to be dynamically allocated and, in order to address the changes of the states provoked by the inclusion of new services, this level engine provides an additional memory characteristic. While the transition tables store the state changes in the main memory, after a new FSM event is received, the engine updates the transition tables in order to take into account the new activity progression. In addition, when a new state is reached or disposed, the engine selects the next state following the transition table rules. This engine has to be able to execute all the required activities that a certain XML description contains, as well as execute the described process of the execution phase. This problem is not covered in this Thesis, but several previous implementations can be used to fulfill the requirement.

FSM descriptions received into the supervisory engine are sent thorough a communication interface properly reserved for signaling communications, avoiding reutilization of the main data path. As these FSM descriptions are often generated in a remotely site, the supervisory engine needs to verify the correct activities 
correspondence of the site infrastructure. To transport these descriptions, any serializable technology can be used, although I recommend JSON or Amazon States Language technologies.

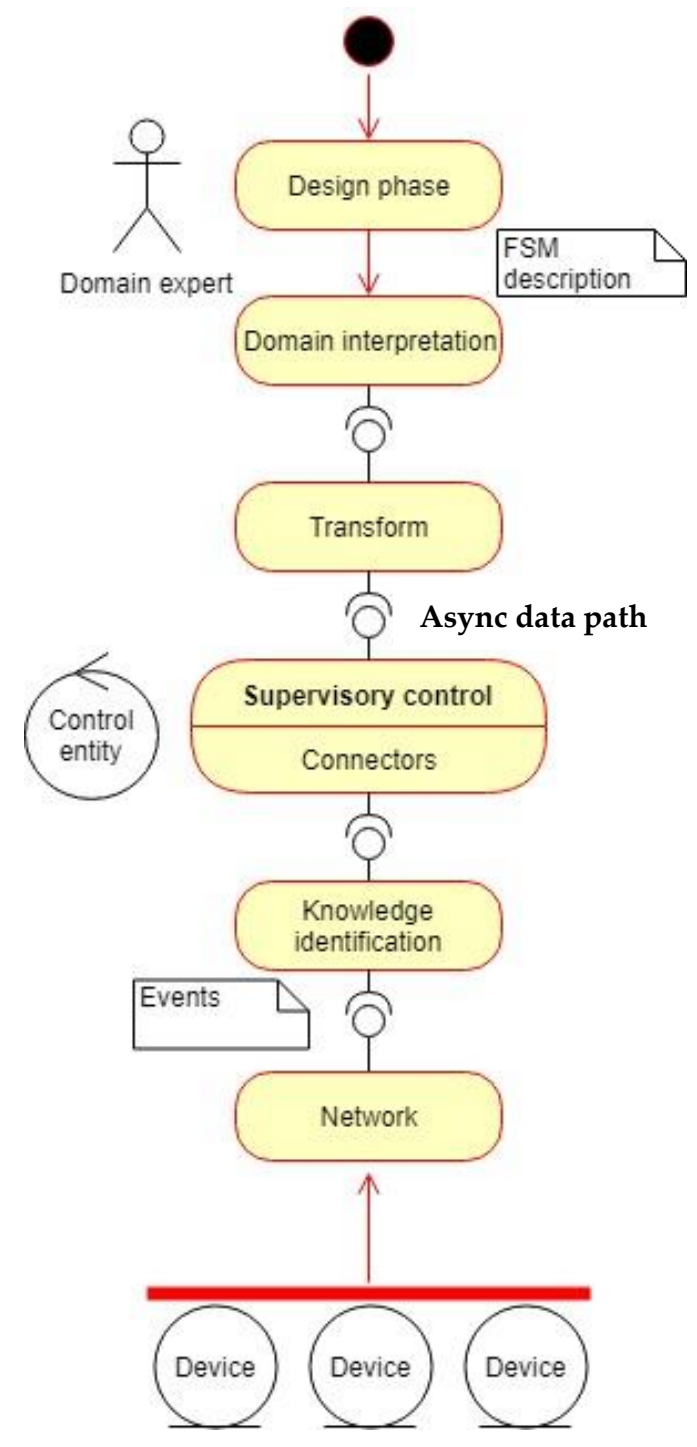

Fig. 15. UML description of the service path

After the decision-making level, where the descriptions are processed to obtain the executable code understood by the system infrastructure, the next level offers additional procedures to a successful task identification chain. Specifically, a knowledge extraction module that provides the control environment to the data acquisition process is added. By identifying patterns, and implementing modeling techniques and machine learning technologies, a complete adaptation of the process to any considered scenario can be achieved. In order to interact with the remaining modules situated on the underlying layers, a publish and subscribe network $(\mathrm{P} / \mathrm{S})$ is proposed. With a $\mathrm{P} / \mathrm{S}$ network deployed, a layer of abstraction between the hardware platforms is situated and all the communication normalized.

This improves the communication between the layers in charge of executing processes from the verification layer, which sits on top, and therefore all modules that 
are dependent of the platform can be integrated in an interoperable manner to the service chain proposed. Several P/S networking technologies can be used, although in most of the scenarios, MQTT can be included.

$\mathrm{P} / \mathrm{S}$ network allows using events as triggers of the execution procedures. The network in this stage is in charge of relying the hardware controller to control the final devices and to gather monitoring information. The hardware controller is the last module of the service chain as they offer the control plane and the connectors to the final sensors and actuators that perform the executions requested. Is in this step where the service defined in the upper layer starts to execute. Depending on the number of final devices present in the infrastructure, and the location of the systems, several hardware managers can be deployed. These modules can perform a primarily inspection and verification of the data received by the devices, or can be simply transparent modules to gather relevant information of the hardware layer.

\subsection{Requirement gathering among real deployments}

To achieve a successful deployment and integration of the novel platform presented in this document, the expertise acquired in previous work and the research provided in this PhD Thesis is used to define and to create a set of requirements. This helps the collection of all needs that a final management system, as specified in Objective \#3, require in a real-world situation.

Following, along with each requirement, a short description is provided in order to clarify and establish the future needs of a standard framework that fulfills the required objectives. Related to the requirement gathering, although more requirements can be identified in a real-world deployment, the following requirements have been proposed as the most important in order to include several service functionalities into the system, as described in Section 2.

Requirements are specified and described with a short description. They are related to the specific objectives defined in Section 2.2 of this document, which were proposed by the motivation of building an extensible CPS framework and a then, with the lack of detailed methods confirmed by the research performed in Section 3. With this knowledge, several functionalities have been observed as the requirements for this environment.

REQ\#1. Automated device identification: in a management system, a reliable identification of the final device is a major requirement to provide precise events to control and communicate to the system. As in our case scenario the devices or entities can be virtual, the process of creating and assuring a unique identification is critical for a successful implementation. The devices will be identified by an automated methodology and assigned an identifier or unique identifier, as proposed in the bootstrapping method (Section 6.3) of this work. 
REQ\#2. Gathering of entity capabilities: to give a proper computing capacity to a device, a characterization of the properties and capabilities offered by the component is required to allocate the correct resources. Capability gathering implies that a list of predefined capabilities must be assigned, and thus, a configuration process has to be carried out. Once the system is initialized, the management and exploitation layer is able to build an available resource schema with all the gathered information.

REQ\#3. Group characterization: in complex system environments like smart management scenarios, some entities by themselves are not capable of performing any substantial process nor even smart enough to be able to communicate with other elements. In this case, aggrupation is made to encompass one or more entities in one device that can be recognized by the management system. This grouping can be done recursively, as the devices can be part of a superior level device that is in charge of their dependent operations. Thus, a device can be composed by a single entity or a group of entities, in a recursive way.

REQ\#4. Group interoperability on all levels: as the system will be deployed on several site-locations, each one unaware of the rest and mostly in different locations, communication between different subsystems is mandatory. To provide interoperability, the communication among intra-layer and inter-layer devices are used.

REQ\#5. KPI measure capability: as a management system entails, measurements of certain aspects are required to analyze the performance and reliability of the system. The system must be able to collect metrics and provide interfaces to interact seamlessly for KPI generation and report.

REQ\#6. Request optimization: in a multi-layer system, inter-layer requests must be optimized to reduce any unnecessary overhead. In this sense, a valid request can be addressed to a certain device whose upper layer knows in advance its inability to process the request, so this request has to be redirected or cancelled at this layer, avoiding excessive requests.

REQ\#7. Sensing and acting capability: unlike systems composed only by sensor devices, a self-managed environment requires acting capabilities to be present in some devices. In this case, the developed system must be able to select and command actuators and verify the correct state of such device to provide feedback into the system.

REQ\#8. Security and privacy: every modern management system has to take into account the security implications as a cross layer that involves every component of the system. As the system is composed of several subsystems, each one must have its own security measures in addition to the global layer that covers the whole system. In this work, security is omitted as the authors rely the security mechanism on the selected framework and protocols. Even, the semantic addition does not modify the security implications of the platform. 


\subsection{Conclusions}

CPS service provision has been proved as a challenge step in which several actors must be implied. This requires a flexible and an extensible process that allows a solution to bring service declaration into the devices in a successful way. One of the main problems when working into the service level, is the need to translate the service declaration (described in a high level) into the machine understandable language that the final devices will execute. In this regard, this section has provided a methodological process in which, with the help of translation engines and pragmatic language, a template generated in a high level can be translated to the device level in an automated manner.

By using hardware components in the service deployment chain, the FSM described in the first stage of the process can be interpreted to extract the domain characteristics of the system. This stage must require the assistance of a person, the domain expert, in order to configure the system and adapt it to the specifics of the domain. Once the transformation is performed, a supervisory control, by means of a control engine, is in charge of relying the FSM processed description to the channels assigned, to allow the production of the events required by the system to work. At the last stage, the devices interact with the system creating a network that translates the information into knowledge used by the supervisory engine, and a technology based on publication and subscription network is proposed.

With this process, a more in-depth understanding of the service provision process has been acquired, and the objective of this PhD Thesis work of providing a framework of automated service provision in multi-site deployments is contributed by fulfilling Objective \#2 and Objective \#5 described in the Section 2 of this document. This understanding of the global process has also achieved a description of the global requirements that a CPS must satisfy to provide the methodologies that this work will incorporate.

With this, a list of requirements has been also described, completing in this way the Objective \#1 stated in this work, bringing a base to any CPS in which several capabilities must be included to allow more service provision processes. 
4. SERVICE PROVISION IN UNATTENDED SYSTEMS, METHODS AND REQUIREMENTS 


\section{Model and reference architecture for the supporting elements}

This section describes the model and the reference architecture proposed in this $\mathrm{PhD}$ Thesis, because of the analysis of the previous chapters where the state of the art, requirements and the proposed service model are discussed.

To provide a successful design of the several components that the architecture model design have, it is mandatory to follow the requirements imposed by the objectives declared in Section 4, where I define what the system will be able to perform, how and what results are expected from it. Summarizing the objectives and requirements defined, the architecture will have to manage, coordinate and monitor several so-called sites of isolated networks in which a CPS will be running.

The services will execute in each site by relying the partial results to a superior layer in charge of unifying the operational results and providing them with the expected parameters required to perform the task in a successful way. These elements are described in their functional way and I only show explicitly the inter-relationships that are required in each layer. This will allow us to obtain a global perception of the system itself. Components often require access to other resources and this is also taken into account in the proposal of the architecture.

The identification of functions, execution controls and execution optimizations in heterogeneous environments involve several modules that takes place in the process. Such as, the state of the defined capabilities in each final device, identify if its state is the appropriate one in the execution context or the reusability factor possible in that task, in order to optimize the general utilization of the platform. In this PhD Thesis, I first propose an additional layer to granulize the execution environments by the incorporation of the called "sites". After, a characterization process in which not only the devices, but also the execution service can be identified to share resources between several services of the system, by using unique identifiers for each entity. I propose the use of identifiers, besides to identify the device itself, to know the status of the device in the face of a service execution. 
One major drawback of using a predefined method to implement an algorithm to identify available entities in a certain system is the inability to predict future modification of the deployed algorithm. This is the basic approach of actual studies, where identifications are stored in a constant updated dictionary. I complemented the identification data with additional metadata to provide the more detail, such as the necessary information of the executed service. This dictionary allows, with the correct algorithms, to reduce the load of the active services by reutilizing some of the required data delivered by the final devices. Recent studies stated the limitations of cyberphysical devices when they are integrated into a running environment, as they cannot identify similar behaviors that could not comply with the defined Quality of Service of the system.

In this contribution, I assume an increase of the power capacity in newer devices that it can be consider nowadays as full power computers but with very little energy consumption. This is why it is of interest to incorporate some of these devices to the architecture, for example, where before a simple gateway could only manage the connection process, newer devices can enable the workflow of the other components, with less power. The incorporation of these devices helps the distribution of the management chain along the system and play an important key managing all other components at they charge. This is why, in previous works, the problem was focusing majorly in the complex devices is the inability of controlling the lightest resources of our system, and nowadays these devices can perform very complex task that can overrides the capabilities of the system major components.

\subsection{Reference architecture}

CPS are composed by several devices that often are in an immutable state or stablished in a certain manner that it is complicated to be altered. In that case, a device model can be defined in the planning phase of the CPS. This strategy involves a predefined model that can be pre-configured or not with the device, declaring its functions, capabilities and requirements in a regular mode. On other consideration, devices can be added to the system and removed dynamically, by decision of the administrator or by external factors that prevent the normal operation of the device, such as connectivity problems, battery discharge or even device malfunction.

The study approach of the multi-site CPS paradigm can be done focusing of several aspects, but the implementation of a universal method to provide seamless integration must cover every component of the user-device chain. Thus, it is important to develop a modular integration to allow future extensions and modifications that allow an evolution and an adaptation to the future CPS implementation, as well as support the current processes that this work proposes. In a primary approximation, I provide a basic model categorization to characterize the different function types found relevant in the literature. 


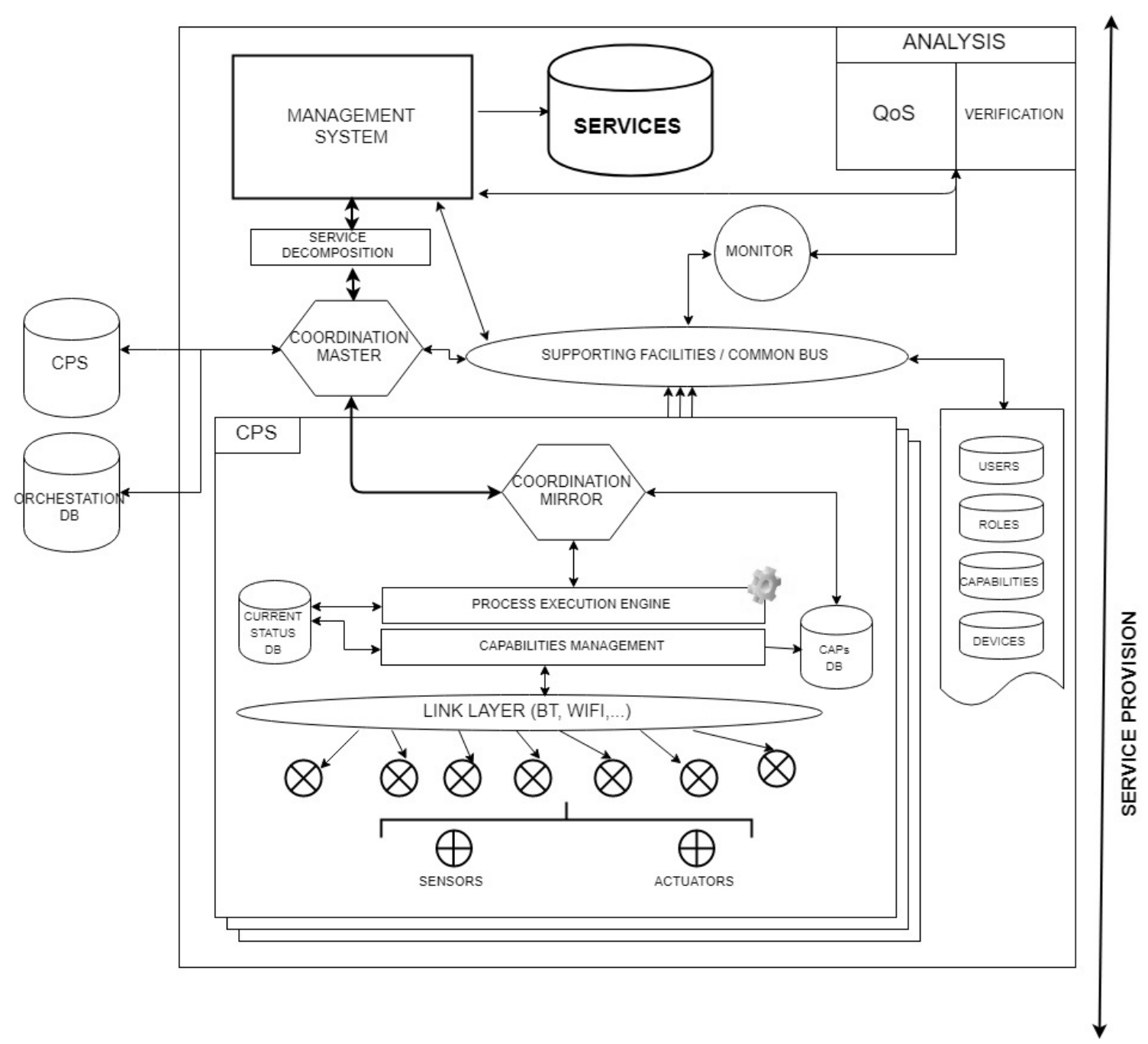

Fig. 16. Reference architecture

Our infrastructure differentiates several abstraction levels. It is so because the system integrates a complex provision framework and doing this separation allows a granular identification of requirements and optimal strategy definition. In a real world CPS it can be found several standalone devices acting as a computational resources that provides information and relies it to a more powerful and interconnected elements, which process it and generate useful information. In our infrastructure, considering the overview presented in Fig. 16, several components are depicted. In this first representation, only the main function and its main database (where available) are shown.

The presented architecture has as purpose the integration of several multi-site CPS that are controlled or managed by a superior entity. The sites in that figure can be seen as different planes, each of one with a coordination mirror inside, to be able to interact with the central coordinator. Three main blocks are differentiated in order to obtain the general architecture layout. The CPS block that includes the sites, the supporting facilities with the added function components (coordinator, monitor and analysis), and the management system along with the GUI environment. 
The reference architecture is presented and divided by the functionality that offers to the system. In the next subsections, each functionality will be described and their components defined. In the description, the functionality that the component realized is described along with the relationships to other components. At last, the database requirements and the interfaces and protocols used will also be defined.

In Fig. 17 a more granulated architecture can be seen, with an in-depth presentation of each module components. In this Figure, the paths of the information transferred between components are shown. More specifically, the control and data planes of the information is presented. An "other" classification of the path is also defined, leveraging the rest of the communication that not fit under the previous types.

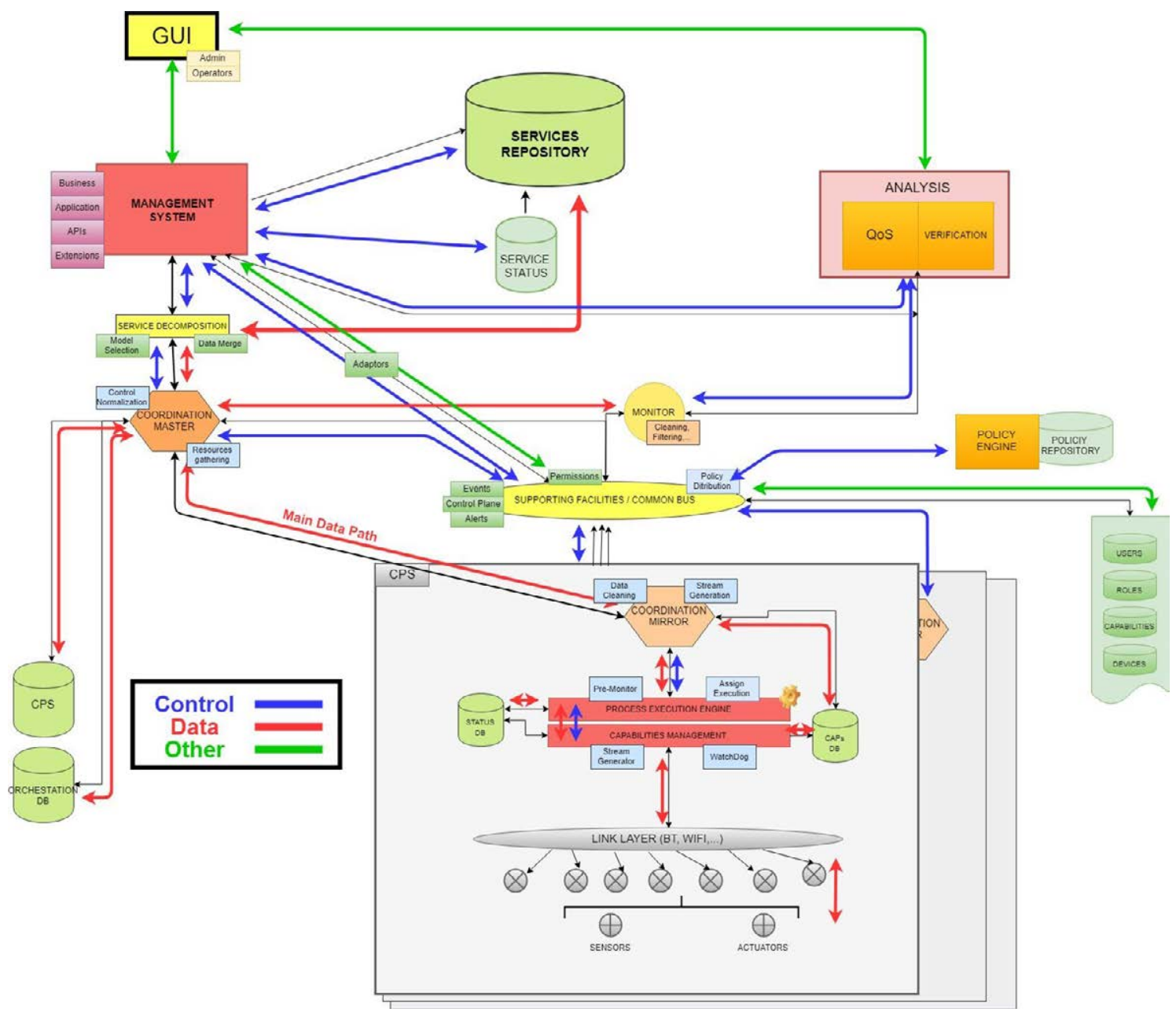

Fig. 17. Detailed reference architecture

With this proposal, an architecture designed for supporting the service lifecycle is defined. As this Section 5 refers to the architecture definition, more specific functionalities of the architecture that are part of this PhD Thesis work are explained in the Sections 6, 7 and 8. Specifically, the new devices auto-provision methodologies will be presented in the Section 6, monitoring the service process is presented in Section 7 and some considerations and energy saving proposals regarding final devices are presented in Section 8. 


\subsection{Management system}

A management system entails the entity that that takes into account the global process of every aspect involved in the subsequent devices that are part of the infrastructure.

In the presented system architecture, as discussed in the previous Section 4, the importance of the uniformity of the data that is produced by the system is the key to provide trustiness to the system, hence, the different interaction between the on-site locations (sites) of several CPS are treated here. This, indeed, requires a complex task of unify the results of several sub-systems executed at the same time and often, to perform related task that in the future will be recompiled and presented as a one final operation result.

As part of this system of the architecture, the user interface will provide a practical way to interact with the system as a whole.

\subsubsection{User interface}

To begin with, user interface or the commonly called GUI (Graphical user interface) will allow the Admins and operators to work with the tools and obtain the results that the services will provide.

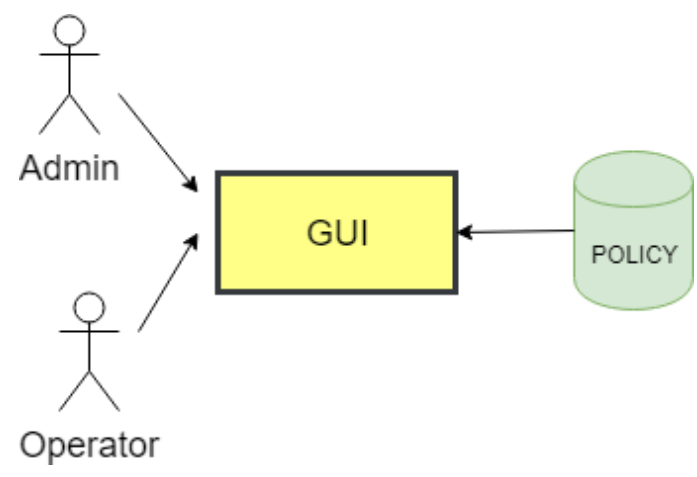

Fig. 18. GUI Use case

It will require the definition of the roles and use cases to offer the correct endpoints to each user in the system. As this first approach, I am considering two types of behavior, Admin and Operator, with different access both interacting with the system as shown in Fig. 18.

\subsubsection{Management environment}

Every system is managed from a central console, this central console (that can be colocated or in a cloud environment) is the central administration endpoint for the configuration and to supervise the operations. It is composed by the management system and a supporting facilities interfaces for enable a central communication bus.

- Management system:

This is the primarily management level. At this level, all the service definition and rule provision is made in order to require low-level entities to provide the necessary data to obtain a result. Actors involve at this level are the knowledge experts that configure the rest of the infrastructure. A master entity that rule the entire 
system is needed in order to maintain control over the rest of the devices and gateways. In this paper, service provision level is defined as a transparent layer that is present and the service plans generated by this abstraction layer are relied to the gateway interconnection to provide the necessary detail of the required devices.

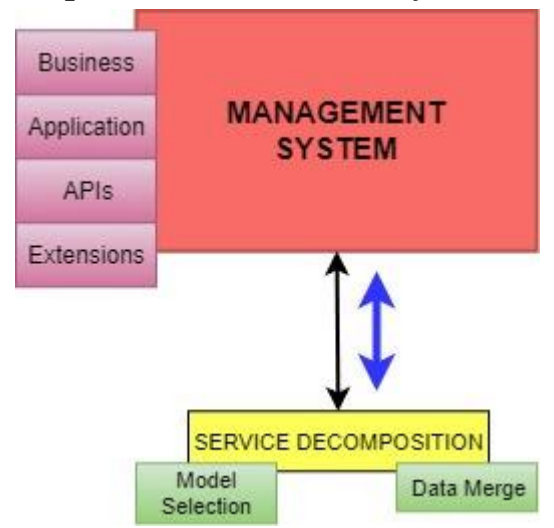

Fig. 19. Management environment distribution

The global entity that controls the available services incorporated to the system. As its main function is to generate and report the results of the running operations the user interface interacts directly with it and provides the external data and parameters required to configure the system as a whole. Inside will reside for example the interfaces connecting the architecture with business logic models, applications or extensions that could be required in a controlled deployment. This can be seen in Fig. 19.

- Services repository:

The general database that stores the services, those previously identified at a precise time in the global system that has been mark as available. The database is accessed by the management system as it is populated by the services acknowledged in the lower levels. This database can be seen as primary global service collection where the final composed services are stored, and not the granular decomposition of capabilities that are later translated and paired to the gateways and subsequently to the final device.

- Service status:

A supplement database is added to the schema in order to separate the actual execution status of the running services. This allows to distinct between control data and actual data that is generated from the different elements incorporated to the system. This data is stored in this database and linked to the service requesting it.

- Service decomposition:

In order to interact with lower levels and to be able to distribute and divide the services into the capabilities required from the final devices, a service decomposition block is placed as the main access enabling the actual communication and data relying from and to the elements. These tasks are important in the data path, as they need to be merged and selected to fulfill upper layer requirements. Decomposition module 
also interacts with the services repository database, as it needs to know the properties of the service to allow the gather of the required capabilities.

\subsubsection{Supporting Facilities. The common Bus}

To support the subsystem interconnection with the common infrastructure, I propose the addition a supplementary entity modeled as a ubiquity communication mechanism available for every module of this and the superior layers. The communication is based in loosed coupling and high scalability protocols such as MQTT to provide permanent availability and reliability of the global system. The incorporation of this module allows the control data to be decoupled from the main data path and will provide the chance of integrate several services such as monitoring and even analysis or verification process without interfering the data path.

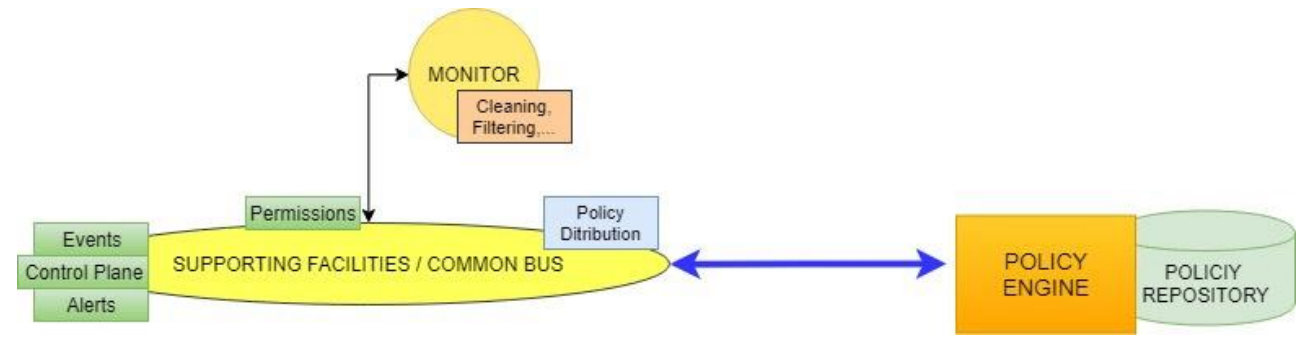

Fig. 20. Supporting facilities

This level aims to provide a context-aware domain in which the devices can be deployed to the system. The context that a certain gateway generates is isolated from other contexts but can be integrated with them in the service provision level.

Gateways can offer several interfaces for different network technologies in order to devices can select the appropriate method to connect with it.

The proposed model abstracts the requirements of a certain technology to a common interface in order to reduce the complexity of the system model. This is done by incorporating a coordination schema between all the several device subsystems that can be part of a same management system, as explained before. This level functional model as represented in Fig. 20 performs several coordination mechanisms to interact with the management level by passing off the relevant information to the upper layer, and expecting the service and capabilities request data as a result of the anticipated process done by the superior level. This service plan contributes to the gateways by requesting the concrete capabilities and results that the devices connected are be able to provide.

\subsection{Coordination engine}

Our first entity in the system is responsible to gather and monitor the data located in external databases. It is called "Coordination Master" as its main role is to communicate with the databases and to manage the interaction at the boundaries of our system with the external mirrors. Information received in this entity will be processed and monitored to detect any new changes in the query results. This can trigger new notifications as the queries are being constantly monitored. 


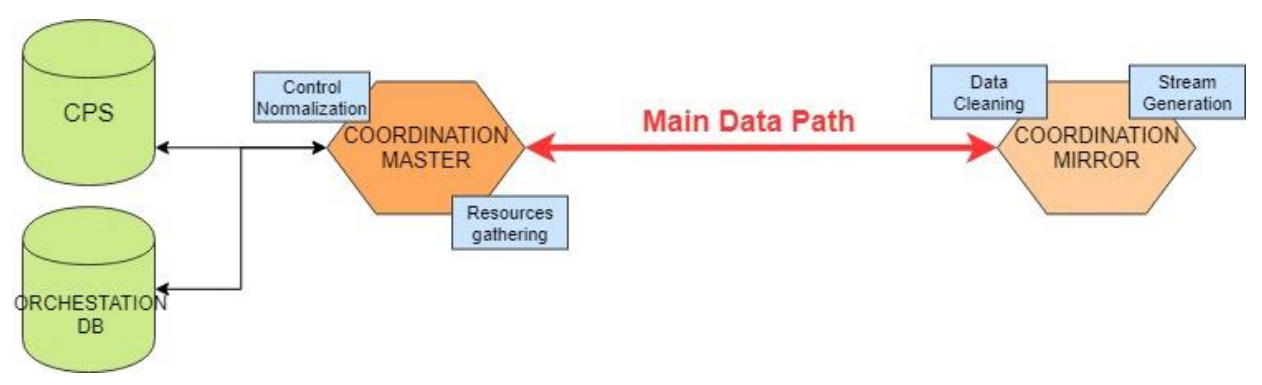

Fig. 21. Coordination Paths and multi-site data normalization

The connection between Coordination master and Coordination mirrors is also handled by the publish/subscribe network, offering the possibility of adding unlimited coordination mechanism and mirrors by locating several brokers into the network if needed. The relationships between the components are shown in Fig. 21.

\subsubsection{Coordination Master}

The Coordination master enables the ability of interact with several device subsystems, the proposed architecture is served by the incorporation of a coordination module and several coordination slaves. As the system can be increased in complexity, it is necessary to granulate the data along the device-service path. The coordination master takes the responsibility of distribute, queue, discard and also prioritize available resources and capabilities, and it does that by relying certain mechanism to the next explained module.

\subsubsection{Coordination Mirror}

It will be replicated in every existing subsystem and will be in a close relationship with the master. Their functions are beyond the basic gateway of a certain set of devices because of the incorporation of a process execution engine that performs delegated tasks from the master. These tasks are supposed to be performed with the final devices, as it could interact directly with the final capabilities required. Execution assignment, data cleaning and in some cases, stream generators are some examples that are implemented in the coordination slave. It is worth to clarify that the coordination entities are in charge of the real data flow and they are responsible of maintain the integrity of the data generated, this is why this component will interact also with databases present at the lower level.

\subsubsection{Policy Engine}

In order to have an authentication method that provides mechanisms to approve or reject the user and devices interaction, a policies engine is added to the system. This entity is grouped with another database that stores the permission level of all entities included in the system.

All actors taking place in the system will be enforced to gain a minimum granted level that has to satisfy the minimum requirements of the entity wished to manage. 


\subsection{Process execution}

To provide the process execution explained in Section 4 and the service integration objectives defined in the extracted requirements, a backend engine that integrates the results of the FSM compilation into the real device manipulation orders is necessary. The abstracted components are detailed next.

\subsubsection{Link Layer - End Layer}

The lowest level defined in the proposed infrastructure is dedicated to the end user devices connected to the platform. These devices are often from different manufactures that are not compatible with each other's and therefore could be difficult to interconnect the different services or capabilities that the devices offer to the system. The methods of connecting each device to its correspondent gateway is relied to the actual connection technology or in the general case, the connection is transparent to the service platform but there is some components that are common to all de devices providing actual data.

\subsubsection{Process Execution Engine}

Just after the coordination mirror takes into account the required resources that a certain device (or a set of them) have, it is time to rely the charge of monitoring the execution process to this module. This is where the final communication with the devices or actuators is made, and the first entry gate of the raw information into the data path. The process execution engine will watch the execution of the required data gathering requested from the management system, as the services involved, and the actual capabilities used in the devices connected.

- Capabilities management: To help the previous module with the device interaction process, a supplementary service will be used in order to provide different database interaction at the device level. This service can provide device status, current capabilities, quality of service information and additional data that could be useful at this layer level.

- Status and capabilities DBs: In order to provide persistence of the control information gathered from de devices, the lower level include a set of databases close to the execution engine and capabilities management to back up relevant information such as the status of the execution processes, active connections, past and current events or the capabilities offered by the devices.

With these modules, a multi subsystem cyber-physical system can be executed and managed from a single user interface, in an easy and summarized point of view. This architecture is the start point of our proposal to add special and unified services for a reusable, optimized and self-managed cyber-physical system framework.

\subsection{Analysis and monitoring}

Analysis, verification and monitoring the service process along the whole system is one of the most important aspects in a CPS system. This element ensures that the correct 
work is happening in real time and in the form that has been conceived. Section 7 focuses on the model implementation, considerations and how the approach is supported into the architecture presented here.

Monitor a process entails the monitor in every state that that certain process can be found in. Consequently, a study needs to be performed in order to discern the parameters of this monitoring and adapt them to keep a reasonable quality of the system.

An important aspect of the monitoring framework as presented here is the type of the information produced by the process, which is indeed relevant from the administrator perspective. As the information varies, a differentiation has to be made in several types. Different levels of monitoring allows selecting and limiting the appropriate information rate received by the monitor system, in order to be able to process the data that is being generated by the co-location environments, a simple way to filter and pre-processing the important information allow having a precise feedback of what is occurring in the system in any case. To avoid failure cases such as a broken communication path or a permanent isolation of a site, the system is provided by an additional bus that can be used to rely selected monitored information to be sent and to reach the coordination master or the remote process interface, where the user interface can be notified.

Often, the scenario of a multi-site CPS present a wide spectrum of devices, in most cases, small factor devices that impose several particularities to the system. Regarding the number of devices, the number can grow unexpectedly, and the mobility is often improved. The use of communicating services along the system cannot be predetermined in a sporadic manner, and a continuous data flow is expected. This case forces the use of a data analysis strategy that in combination with a data monitoring process, provide an extensible CPS environment. In this sense, this PhD Thesis work proposes the use of the QoS KPI (Key Performance Indicator) as explained in Section 6 to allow a granular capability selection and identification mechanisms that with the incorporation of the monitor system described in Section 7 grant a flexible CPS deployment in any case.

\subsection{Coordination layer control}

In this thesis work, I consider the coordination element of an on-site CPS subsystem as more than a simple function module as explained previously. By using the incremented processing capabilities of the gateway device, the system can rely certain functions to the middle layer and release the service creation from the granular management of the devices. This helps the creation of more complex infrastructure as more on-site subsystems can be incorporated at this layer.

This is why, besides the behavior of the service interconnection procedures, the middle layer also acts as a trigger mechanism for the final device in which the actual data provision or is made. Due to the fact the devices can be part of several services at a time it is needed an entity capable of indicate them their coordination entity in which the device is located. 
Implementing a middle layer that interacts with devices and takes part in the service recognition process can be a challenge task. Especially when the possible devices are heterogeneous in a form that it is difficult to model in a unique way.

Thus, the gateway layer defined in this thesis performs two main tasks. The first procedure that the coordination layer provide, is to homogenize the connection interface by offering standard methods to interact with connected devices. These methods must be available in each device and they have to be supported by the hardware and implemented by the software.

Information interchange occur at the beginning of the connection, the device must inform the coordination slave of their status, including who is, how is connected and what capabilities have. As I have already defined, this data contains the necessary information to be sent to the gateway in order to start the new device addition process.

Second task assigned to the gateway layer takes into account a successful new device inclusion. This implies the start collecting every information offered by the device and generating a system status to update the state of the context of the system.

In a top-down description, the on-site coordination entities presented here, are in charge of rely the information received for their devices to the management system to the process execution engine and the capability management modules, which with the method described in Section 6, a system update is performed. This component has to declare a scope directory for each service provided, by incorporating a unique identifier to this service in a precise context, this way, the coordination layer can know the lifecycle of the execution in each device.

Additionally, when a service execution needs resources of a known device, it triggers a new request message from the coordination layer, which is in charge of the required knowledge. The control layer then, controls and manage the final device by requesting the current expected behavior and manage the concurrent services that could be executing at the same time and using the same resources in the final device.

\subsection{Summary}

This section presents the reference architecture for a multi-site CPS environment, based on the previously defined requirements. Using a component per function approach, the resulting system is able to identify all the actors and modules integrated into the system and to perform task that are created and launched from a central management entity. With this proposal, I try to accomplish the Objective \#3 stated in this work by the incorporation of different functional modules focused to incorporate multiple on location sites support and favoring the extensibility of the system.

Regarding the service execution model, this section focuses on the coordination of elements at the data plane (transfer of information produced by users or Web objects to other terminals) and the control plane (synchronization and management of the execution flow of tasks and activities). The interaction between services is resolved by 
introducing the necessary interfaces between the modules that process the activities, based on workflow patterns. The channel creation and optimization contribute to the communication establishment between limit activities, taking into account the arrival of new devices and the problems that can be found in the communication layer. Finally, the coordination layer is described by the interactions between the different modules of the defined architecture, in an environment with multi-site locations and taking into account the possible node characterization and link loses. These modules and procedures fulfill the REQ $\$ \mathbf{4}$ by providing interoperable mechanism between the system components.

After, I evaluate this model and analyze the improvements of the described optimized architecture implementation and compare the results with the inclusion of the monitoring system model described in Section 7. The performance evaluation of this work is provided in the interoperable device testbed described in Section 9.2. 


\section{Methods and functions to auto- provision deployments}

In this section, I propose a methodological service propagation technique that allows simple hardware entities present in the system to provide its capabilities and to enable the system to incorporate new resources in the workflow of their execution.

I focus on how novel methodologies can offer auto-provision deployments in the architecture presented in Section 5. Auto-provision refers to the mechanism that enables automatic service integration in the system as is. Some auto-provision methodologies can often include several processes in the lifecycle of a service, such as auto-discovery, auto-recognize and auto-configuration of the devices and entities that forms the integrated system. Thus, the newly incorporated devices will need basic manipulation in order to gain the execution privileges that the system require to process services. This section models the presented system as a service-based design that includes domain experts into the loop to allow final users access without the need of acquire further knowledge. This is translated into the following contributions:

- Based on a three differentiate layers, several integration phases are declared. A workflow is described and based on a generic service the information chain is processed from the hardware layer to the business level. At this point, data is generated and attached to the service description and QoS requirements are met into the process. A cost model is used and the weights calculations methods are declared.

- Several components are defined to help the realization of the device incorporation workflow.

- A bootstrapping method is proposed based on the use of a template sheet predefined by the domain expert and optimizing the re-utilizable capabilities of the devices present at the integration process.

The section structure is as follows: Section 6.1 defines the service-based model used in the architecture description of the proposal. Section 6.2 review and explains the different 
conditions that a real deployment requires, along with the relation of the identified requirements and with our component model breakup. Section 6.3 presents the fast-self configuration solution that I propose to integrate newly devices in the system and the component description and its functions. Section 6.4 adds the bootstrapping method that introduces an algorithm to identify the capabilities of a device in a new provision framework.

\subsection{Service-based analysis of smart environments}

To analyze and overcome the service-based environments providing an interoperable service facility, an infrastructure called Smart Environment (SE) is usually defined (Youngblood, Heierman, Holder, \& Cook, 2005). A SE is an environment that is able to acquire knowledge to apply it to the same environment in a feedback way, in order to ease and improve the overall experience.

I this manner, the traditional smart environments are often seen as monolithic compounds where the integration of the all layers are defined in a strong manner. Is in these systems where the operations are described as predefined loops where the state of the environment is sensed, then the reasoning is performed with several rules that has been previously identified and then, an action is performed with the intention of changing the started state. This is why it is normal to say that the environment perception process is a bottom-up process (D. J. Cook \& Das, 2007). Every component of the system is indeed dependent of each other using this view (e.g. an objective must be measured with other underlying hardware devices), and the domain experts has to be incorporated not only in the design phase of the system but during its normal working operations (Alcarria, Robles, Morales, \& González-Miranda, 2012). Integrating the objective of obtain several uncorrelated elements in a service-based environment and allowing actors with no technical knowledge to interact with the system is a new mode to approach this scenario.

Designs that are abstracted into Service-based schemas are currently very favorable (Jammes et al., 2014), as various levels of abstraction are defined as independent of the different context and may be employed in such different ways. The encapsulated services can be transferred with easiness, and new applications and system can benefit their inclusion. This is why, the economies of scale are improved, and then the SE deployment is encouraged.

With this introduction, as the approximation to de problem is now service based, traditional service operations are willing to change. In particular, the discovery process of a new device has to be taken into account, as the services situated in the low level of the platform needed to be aware of such changes. Hardware incorporation is indeed processed, and the capabilities transformed to the upper level in the platform. Seeing from a perspective, the service environment operation is described as the publication of the available services from the hardware level and the central server acknowledges the process by using a transformation process. After that, the application level, controlled 
by a user, requests the central server asking for the required capabilities to gain the new services. This is when the system managers can use the selected services to provide the user a more gratifying experience by declaring rules that the users can apply. The application layers can now monitor the state of the execution and the environment. With this approach, the smart environment maintains the bottom-up perception wanted, and in the same time, it is independent from all the layers involved in the process. As another advantage, the system is not required to use special software of heavy-duty machines to operate.

To apply a service-based architecture has another advantage that is the social implications that the humans can provide to the system. In this approach, humans can be seen as service providers (D. Cook \& Das, 2004) to the system. To define the human implication, a "service provided by a human" in this context is whatever action performed by the people, as a request from the service environment. To provide an example, in a certain time of the execution, the system can ask a certain person to provide an action, through a mobile application or an in-situ mechanism, to "close a door", in order to maintain the temperature of the room with in stablished margins. Then, it is said the service "close a door" is provided by the social entity. Usually, researches on the relation between social entities and social environments are focused on monitoring the behavior of themselves or recognition of activities performed by them. In this way, social entities can be seen as "consumers" or applicants for system that need certain actions - that cannot be done with only machines- to complete the executions. Also, there are projects like MavHome (Youngblood, Cook, \& Holder, 2005) (Managing an Adaptive Versatile Home) that have introduced social entities in social environments to perform various roles. This presents another problem knows as "humans-in-the-loop" (Munir, Stankovic, Liang, \& Lin, 2013). Fig. 22 presents the proposed schema for a service-based SE. It can be seen, social entities of the system appear in the top part of the figure as services consumers, and also as service providers in the bottom part of the architecture.

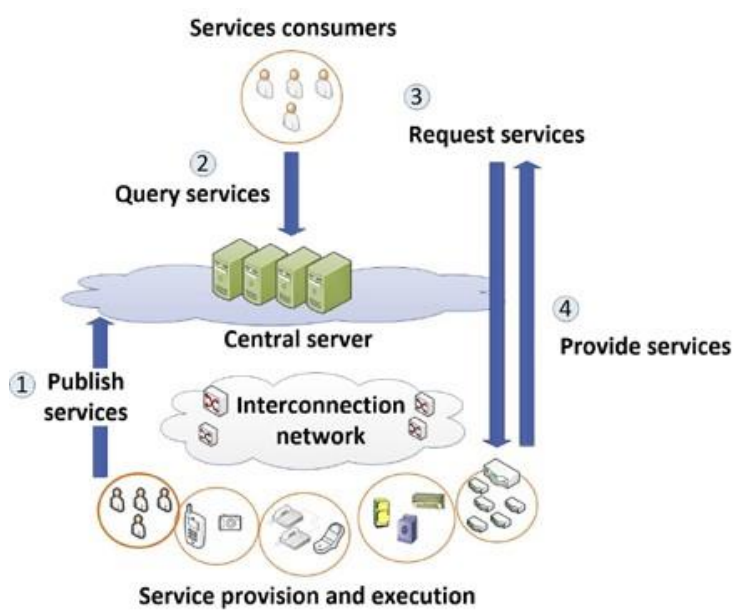

Fig. 22. Service-oriented Smart Environment scheme

However, systems that are service-based can come with unexpected problems and challenges. In outdated service environments that are based on feedback loops, the infrastructure is designed to react to the stimulus with anticipation to the physical world 
state changes, as implied by stability criteria. With solutions focused on service-oriented premises, the temporal requirements can be preserved, as service environments value increases if real-time operations are supported (De et al., 2012). Nevertheless, these systems often need the people to perform the transformation process from low level services into a more comprehensive abstraction into the high-level service set. This was imposed by the need of understand the means of every service that the service is in charge .(TRAN \& TSUJI, 2007) Then, as it is a manual process, the completion of realtime requirements cannot be executed in the operation time, because the cited abstraction is very consuming. To overcome this, the service definition is defined at the configuration time, and the different levels start with the configuration of the services in an immutable state through the whole operation, or until an expert updates the states. These changes in the physical layer of the platform are often treated as service changes that can occur in the same way that in traditional service environment, and so, these services configured by domain experts are not available to configure during the execution of the environment. The limitations are imposed by social components and mostly by hardware components.

To provide a solution to these problems, I have proposed two procedures that help the transformation phase. In a first place, and knowing that there are solutions to try guessing the availability of a certain service with no need of a previous invoke, I propose to break the abstraction layer independency between the service based implementations, by providing the information of the hardware layer, to the high level components. Additionally, the process implied in this solution can be exhaustive, and real-time computations can be compromised in a social environment (Charif \& Sabouret, 2006).

In second place, new applications can be depicted from new hardware included in the system, so the services can often request new functionalities as they are included, but also the devices can refuse the execution of a certain request. If this happen, a process to make high layers aware of the rest of the system is needed, with the final objective of take into account the new system and be ready to fulfill system requests. This can be an iterative process with real complexity, so it may reduce the support of real-time processes (Alcarria, Robles, Morales, López-de-Ipiña, \& Aguilera, 2012).

\subsection{Fast-self configuration for interoperable device integration}

To overcome the Objective \#6 presented in this thesis, a solution to provide a way to self-configuring the system for supporting the integration of new interoperable elements is proposed. It is based on three different independent abstraction layers. Fig. 23 shows the general proposed scheme for an implementation of the described method. I have divided the context level in business, production and hardware level. New devices located in the low level must be incorporated to the upper levels of the schema. 


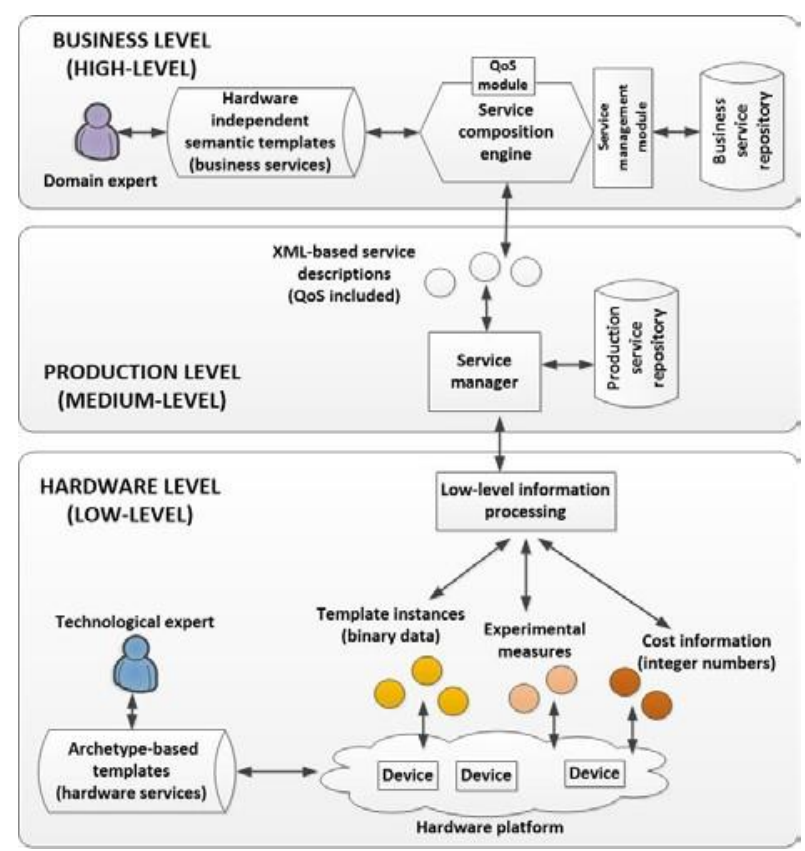

Fig. 23. Reference architecture for a fast-self configuration solution

Following, I start describing the different levels that I propose to use in order to obtain a device integration process. The lowest level is called hardware level, as it can be seen in the Figure. This level is composed by the device components themselves and several function modules. Additionally, I introduce an expert figure that will help the creation process in a configuration phase. This first domain expert is required in order to know the necessary technology knowledge to apply the base implementation process, but can be unaware of the application that this implementation would serve. This expert needs to configure the services that the infrastructure must support and that can be included in the hardware services. These components of course, could be used in a present time or in future moments.

With these services identified and the archetype -an archetype can be described as a pattern or a model that contains all the depicted elements of several copies (Bicer, Kilic, Dogac, \& Laleci, 2005)- templates generated, every hardware device that supports services must be expressed in a form of the identified capabilities, or a collection of them. In this way, a certain capability, for example a temperature measurement sensor, can be reused by several hardware devices. The output of this level as described, is a list of archetypes or capabilities that represent all the capable services involved in the configuration process. The domain expert must generate these descriptions that can be automatically processed and included in the system in the end of the configuration process.

While the configuration process is in place, it is worth to mention that the information generated in this phase can be processed to reduce the spurious data, and to assure that only the important information is transmitted to the other levels. Also, additionally to the automatic information processed, a simple semantic characterization of the services identified need to be filled by the technological expert, and this information transmitted along to the upper level. 
Next level focus on produce the service abstraction to feed the business level. In this level, which is called production-level, services are analyzed, and the devices are abstracted from them. The obtained information is instantiated and a transformation takes places to produce a generic service description output -using XML, as an examplewhere all the semantic information is automatically filled. At this time, it is necessary to left behind all the device communications specifics, so a substitution API is generated in order to homogenize the requests and the answers provided by the hardware level. In this level, all the hardware infrastructure is agnostic and can be seen as a black box with standard APIs to interact with. Then, a repository containing all the services is generated at this level.

In this level, the service description that is generated, is prone to contain services that are not valuable to the users in the service environment. This is where the upper level proposed takes its place, and has the objective of create the needed value for the users of the SE. The business level creates with the help of a second domain expert, the relevant services that will be offered to the users. This expert has to have the knowledge of the specific application and the relevant indicators in order to produce the required templates. This information is produced in a domain description language, containing a collection of semantic relevant templates.

Along with those templates, the description obtained by the lower level are composed and processed in an engine to finally create the collection of services. Those services, well defined, will be available to the final users in the service environment.

The described process, in fact, left several factors that can impact the services in a fully functional CPS, such as the performance of the executed services and their implications in the composition of services to obtain a common result. In this solution, additional data can be produced to align the requirements identified in Section 4 by complementing the service information in the system described. A Quality-of-Service can be indeed identified and calculated in order to be part of the adaptation procedure that is triggered when a configuration process has ended.

In this proposal, the information of QoS can be represented by any system performance indicator that affects a certain service. A numerical representation, to give a quantitative key would be an option, but there are situations in which only a certain value does not indicate a valid indicator. Collections of any measurable entity can also represent that information to allow a wider compatibility and even functions must be allowed to be implemented since the dimension values may require an adaptation process to be really valuable. As QoS is used to indicate the importance of certain parameters in a service execution environment, it can be used to evaluate current values of the system, but other non-technical definitions of QoS are not considered in this proposal.

To provide examples of what classes of information are considered in the QoS calculation, I propose the use of two sources. First, a set of periodic measures that can be performed to network parameters are taken into account. Transmission rate, delay, jitter, 
and several others can be performed into the lowest level and processed. The results then can vary between different hardware devices as they are differences between them. Elements such as time, interferences or device positions can affect the results. These parameters are known to be changing over time, so the evaluation period of the adaptation process is a critical parameter to choose. Second, costs associated to service execution are also a source of valuable information. As this information can be also provided by the hardware level in a continuous way, the cost can be calculated without being request, and then gathered in an automated process. Information such as CPU utilization, available RAM, battery information and etc. are processed to obtain the cost of a certain execution and therefore, impact the QoS information generated. This $\mathrm{PhD}$ Thesis is not focused on the algorithms used to perform this calculation, although it can be easily found in literature. It is noted, that cost information related to hardware devices, hardly changes in a fast pace, as the devices often rely in embedded firmware that are not often changed.

\subsubsection{Device characterization}

In order to obtain a precise characterization of a new device, the first process uses a device characterization process to help the generation of the supported capabilities. This process is replicated each time a device wants to be include in a new system, but also when a device registers a new capability to be used for the final process.

To formalize the method, I define an execution environment $E$ that shapes a Service Provision platform composed by different types of devices. In the model, a device $D_{i}$ is represented by a collection of several $m_{n}$ parameters in the following way:

$$
D i=\left\{\mathrm{id}_{\mathrm{i}}, \mathrm{m}_{1}, \ldots, \mathrm{m}_{\mathrm{n}}\right\} .
$$

The device is identified by its unique identification $i d_{i}$ and a set of parameters in the form of key and value, selected and preconfigured in the device. These parameters, that are a subset of the $D i$ set, can be also identified from the standard messages that default device provide, thus in order to start detecting them only is necessary a first scan or connection with the link layer of choice.

The execution environment then can be expressed as a set of $i$ devices currently connected.

When a device is connected to an execution environment that forms part of a global system management group previously configured, the capabilities management engine as well as the process execution engine are aware of the inclusion and start relying the $D i$ set to the management system.

As stated before, the device $i$ can be part of an on-site location, so the information must flow through the common path in order to be considered by the upper layer. In addition, the device model $D$ could incorporate a device location information parameter to the list and the process execution engine of each on-site location can decide if the execution is assigned for that device. 
Once the lowest level processes the information received, the module analyzes the experimental measures and the costs associated and induces a certain ratio comprising the offered QoS calculated before in a certain range (this has to accomplish the maximum QoS and the QoS guaranteed of the system). Abstraction layer keep the independency between the low-level information layer and the production level, as the first one cannot send its information directly to the second one. Additionally, as the produced data flow can grow as big as the entire system component quantity, the platform can be scaled to be adequately dimensional, and perform a successful design of the infrastructure.

At the second level, the information production process can comprise the QoS information into the XML document along with the service descriptions. All the information is therefore used to calculate a quality value to be compared with a predefined threshold. This threshold can be defined by any of the domain experts and is a minimum quality indicator to decide the status of the service.

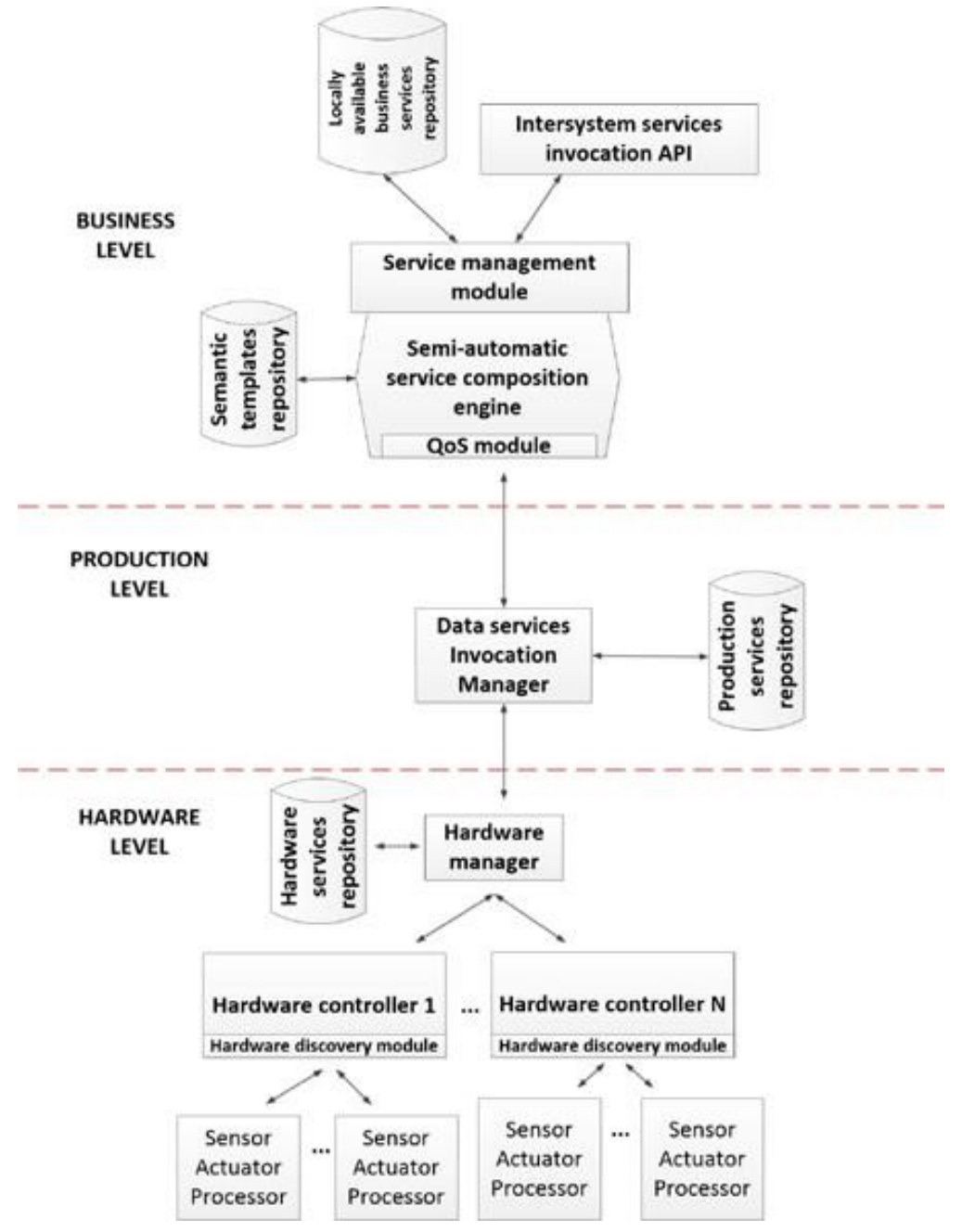

Fig. 24. Functional architecture of the fast-self configuration solution

It has to be noted, that the described process can be repeated when a new service has to be discovered, or a new device joins the infrastructure. In order to include the new information, recalculating the service composition is unnecessary to recalculate the state of a service, because that state only depends on the QoS information. This I why in this 
proposal, the catalogue of the services is updated in a very fast manner, as the most of the traditional service composition procedures are avoided (Zeng, Benatallah, Dumas, Kalagnanam, \& Sheng, 2003).

All the techniques described here have to be supported by several components, performing functional tasks. They will be defined in 6.3.1. There are several architectures identified that could incorporate the proposal, with the necessary components added. $\mathrm{T}$ better visualize de workflow proposed, the Fig. 24 shows a functional architecture of the infrastructure.

\subsection{Bootstrapping model}

Capabilities offered by final devices in a cyber-physical system can be abstracted from the service layer by identifying services that the user can require. This manner, the presence of a certain device in a complex system can be seen as a part of a certain service that can be used and therefore, offered to the upper layer.

In our defined infrastructure, services are formed by grouping the founded devices and capabilities and offering these to the management layer. Moreover, the management layer can request the status of a certain device or information about the reliability of the capabilities involved in an operation, for example, in order to determine the exactitude of a certain process or service. The problem is that with several contexts running at the same time, it causes deviations in the status of the other contexts in an inevitable manner.

To overcome this problem, I propose the incorporation of unique identifiers to services along with devices identification. I define an identifier as a unique identification string or number which involves all the layers that a certain service or capability needs to be identify in a process context.

The identification string will be composed, as before, as a matrix of arrays, using declarative and exclusive elements that define an entity in our system. Table 1 shows the first proposal of the model, but other proposals can be considered.

Table 1. Information model.

\begin{tabular}{cc}
\hline Selected elements & Content \\
\hline Service id & Generated by Service Decomposition \\
Gateway[array] & Mac Address \\
Scope & Assigned by gateway (optional) \\
Device[array] & Device identification \\
Parameters[Dict] & Key, value \\
\hline
\end{tabular}

Service and scope elements are defined by the service provision layer and are replicated along the lower layers. One service in the management system at the top will be available as required by the gateways in the cyber-physical system. Scopes are created at gateway level and it will be enclosed to the directly entities related to that gateway and not replicated upwards. Parameters are gathered of the device itself, and they will 
be replicated upwards to the assigned gateway and service provision layer. This behavior could be modified if needed in future iterations.

With every new created service, a new collection is produced in the management system module, by grouping the selected elements. That is needed to control the amount of resources used in a certain time, and with that, the system can start to test out if any of the previous services already running can provide the same required results of the new service. The service then activates the shared resource engine and in a seamless actuation, the provision of the data is realized without the need of subsequent data request from the final devices.

It is assumed that the management module is aware of every service created, no matter the final user that creates the service so that the share of the resources between contexts is possible. Related to this, a clarification of the shared data boundaries is worth it: the user does not have the exclusive exploitation, but only the device data will be shared, this is, the usage that the certain service will made of the raw data is kept private as the business layer of each service is preserved to a single user.

The service identification will be exchanged along with the capabilities used to permit the sharing of the results generated by the management engine and the set is replicated in the gateway responsible of at least one device implied in the service. This way, the system can user the gateway entity to perform service selection task at the middle layer.

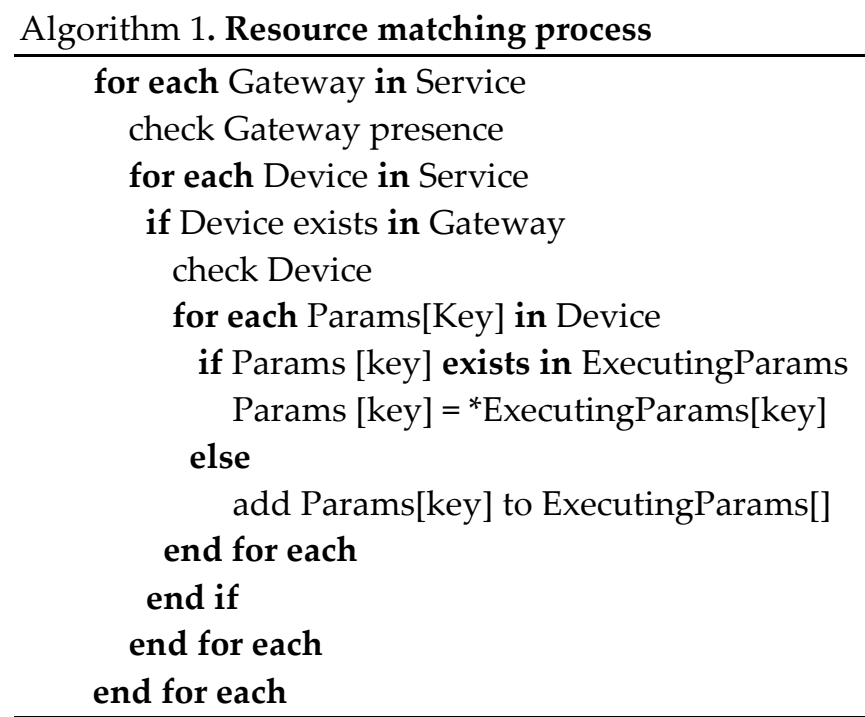

In order to perform the share of the resources, a first test algorithm has been deployed to the management system. The test algorithm performs for every created service an iteration between current running services to discern which resources can be reused and avoiding repetitive requests. The outline of the algorithm is presented in Algorithm 1. Resource matching process. 


\subsubsection{Component description}

In order to integrate the bootstrapping model in our general architecture presented in Section 5.1, it is required to define some elements in the proposed architecture. Fig. 25 shows the element in relation with the modules presented in the general architecture, focused on the hardware level of the CPS.

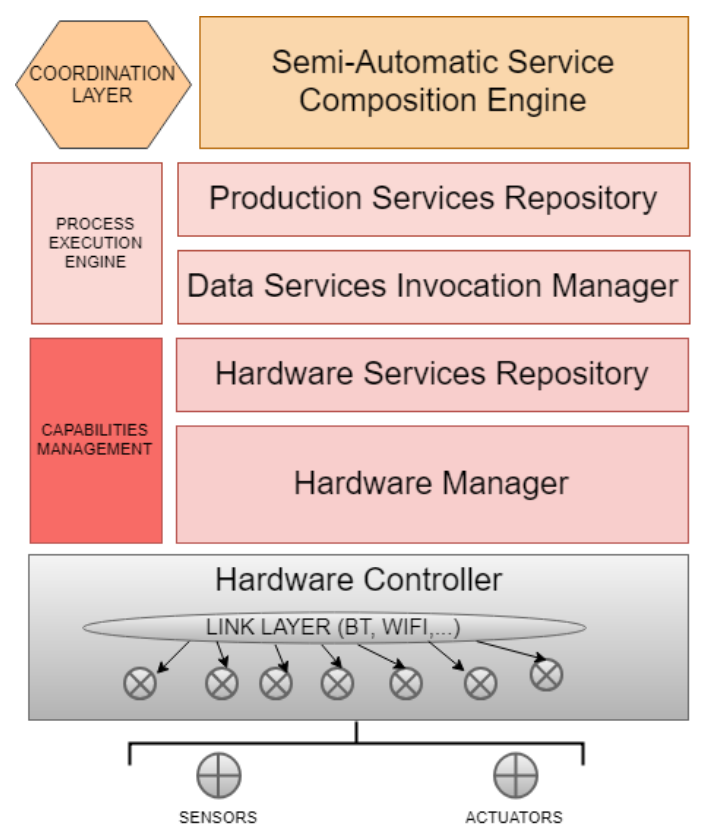

Fig. 25. Bootstrapping elements

In the next paragraphs, a description is provided of each element that perform a function in the proposed architecture. The requirements identified in Section 4 also are related to the component $s$ functions in order to deliver the auto-configuring capability of the system.

- Actuators, Sensors, and processors: Hardware infrastructure, including the final devices that are present in any smart environment. Traditional sensors to the newly Smart Objects (Kortuem, Kawsar, Sundramoorthy, \& Fitton, 2010) can be included in this group.

- Hardware controller: entity responsible of managing the hardware infrastructure. Also, the discovering and configuring processes are carried out by the hardware controller. Its main task in the infrastructure is to gather the archetype-based information that comes from the low level and instantiate them for the hardware manager. The infrastructure can have as many as hardware controllers as the onlocation places requires. As the final devices can be connected with a variety of different hardware controlled, each entity is required to have a hardware discovery module to interact with those final devices, being able to have more than one and capable of interact with several technologies at a time.

- Hardware services repository: it holds the archetype-based templates in order to have all the information related to the hardware services. It also has simple semantic information associated to each service to easy the selection and 
gathering. When available, it also has the generated instance and the master template. All this information will be used by the hardware manager to know the status of certain services.

- Hardware manager: different hardware controller must be coordinated by a hardware manager. It manages the instances reached by the low-level hardware implementation and manages the archetype-based templates. Processing those templates generates a unique master template that holds all the available information in a manageable form. This form will be sent to the hardware controllers to instantiate it. Additionally, the low-level data is processed and submitted to the upper level components. To complete, it also manages the onsite information of each underlying hardware level components where certain capabilities are available.

- Data services invocation manager: it assures the independency of the hardware devices and hardware manager from the service domain. This module offers a translation mechanism from the archetype element template to a generic service instance that is based on standard technologies, and allows hiding the complications associated to the devices in the higher layers.

- Repository of production services: it is a component that stores the set of production services. They are described by the data services invocation manager. Also, it contains the particularities found between the generic interface and the hardware ones if any, so they can be offered to the higher levels.

- Semiautomatic service composition engine: in order to compose the high-level services and the information provided by the data services invocation manager, this component uses techniques that requires human intervention only in the configuration phase. In such way that the domain expert must generate the list of semantic templates that describe the services used in the higher levels. This component comprises the QoS calculation module and the management module that aggregates all the information gathered and decides the status of the service.

At the end, when the available services are defined, they go registered in a repository available to the business level, so the application can call them and execute.

\subsection{Summary}

This section addresses the need of a former method to incorporate services offered by new devices when onboarding a new system. This work is related to the Objective \#6 declared in this thesis and contributes to the global system by supply a necessary mechanism identified in the identified requirement REQ \#1 of this proposal.

After the definition of a smart environment and analysis of the service-based frameworks it is clear that the traditional problems on the SE based environments are depicted by the feedback loops that in most of the cases are predefined and preconfigured. With the addition of dynamic process, the abstraction achieved are more 
complex and more powerful, integrating in the loop the service creation and autodiscovery capabilities proposed in this work. This proposal fits in the group of mechanism that relies the low-level service definitions of the hardware level to the superior levels where it can be managed.

To overcome the difficulties of that kind of process, and keeping the process cost under the required margins of a real-time system I propose the inclusion of the templates stated in Section 6.2, where a technological expert includes the only manual step required in the onboarding process. By the using of archetypes, a template can be generated and repeated for a diversity of devices, as long as they have some similar capabilities.

By the inclusion of the QoS information, the upper layers can evaluate the parameters compatibility that the new device is exposing to the system, and nevertheless, this information can be updated during all of the device co-existence in it. This allows the production level to be aware of certain changes in the behavior of the system, and therefore it can manage the different elements with the information obtained in the information retrieval loop.

To aid the proposed method at the first phase of the device onboarding, a bootstrapping model is proposed to guide the inclusion of the device and to assure the device incorporation into the system. 


\section{Monitor and execution validation model in an interoperable multi- site CPS}

In this section, I propose methods and procedures to monitor and to assess the executions of processes in a system composed by several site locations. The system that manages a flexible CPS with ongoing processes in a multi-site environment was defined in Section 5. The requirements imposed by the Section 4 in relation with the needed feedback are the base line to address a challenge found in a multi-site CPS.

When working with services, the scenario presented at Section 5.5 identified most of the complex cases that the coordination engine needs to be aware of, and trying to discern when the monitored system have to take into account is studied. In this case, the monitor process is understandable as the background process in charge of verifying the correct execution, and completion of a service in the system.

After the Section 6, where I explained the different methods of the devices to include them in the system, in this section I will focus on the always important task of assuring the correct work of these elements. This will be even more critical since the devices has been automatically detected and therefore a reliable monitoring framework will be key for a successful integration.

This section is distributed as follows: in Section 7.1, I propose the monitoring model as introduces the previously presented architecture by using the specific modules of this task. This will guide the former data path along the monitoring elements that are required to provide and gather information in the system. Section 7.2 describes the service state monitoring process as I identify the possible states in which a certain service can be found, categorizing the diverse characteristics and particularities to observe. Section 7.3 contributes to resolve the state-less problem and loop inclusion to the monitoring path, as it may happen when monitoring a large and a complex system. Section 7.4 summarizes the work. 


\subsection{Monitoring model and process lifecycle definition}

In this section, I define the model of a monitoring framework focused on the resiliency of the information and the requirements defined in Section 4.4. Specifically, a monitoring model that allow incorporating several on-site controllers and rely the information required to a central module. To do this, a model of the monitoring framework has been designed and incorporated in the global architecture definition, shown in Section 5.1, and explained in the following section with greater detail.

When designing the architecture of the system presented in Section 5.1, a focus on the incorporation of new devices and having their capabilities recognized by the superior entities was necessary.

In the service process, once a basic working scenario was built, a simple monitor entity connected to the management system was designed. This quickly came up as an insufficient module due the predicted workload, as the system became complex. The main challenge was in fact the incorporation of the so-called sites, which were explained into the Section 5.1. The addition of the different context in which an execution can reside and the possibility of re-use the different final devices in more than one service execution lead us to try to resolve the challenges found in this situation.

As stated before, monitoring a process entails monitoring in each of its states. Consequently, a study needs to be performed in order to discern the parameters of this monitoring and adapt them to keep a reasonable quality of the system.

In this section, device monitoring is assumed as part of the running system, but a focus on the process execution is made. As the process can be abstracted from individual devices in a way that, the process can be executed simultaneously in several and independent final devices.

A most important aspect of the monitoring framework as presented here is the type of the information produced by the process, which is indeed relevant from the administrator perspective. As the information varies, a differentiation has to be made in several types, declared in Table 2.

A first type is the process metadata, where the process is described and classified. This information often incudes common keys that are adequate to filter the information and to locate similar process behavior in the system. As it includes key value pairs, it is easy to discern the type of the process that this information is monitoring. This is where Section 6 proposal, about incorporate unique identifiers to the individual process, could take advantage. Some examples of process metadata information can include identification numbers, public keys, encryption keys, and other information that is not updated frequently. 
Table 2. Monitoring information types

\section{Process metadata Process configuration Process State}

\begin{tabular}{|c|c|c|c|}
\hline Description & $\begin{array}{c}\text { Defines and classifies } \\
\text { devices }\end{array}$ & $\begin{array}{l}\text { Updates a device's } \\
\text { state by sending the } \\
\text { expected state as a } \\
\text { configuration }\end{array}$ & $\begin{array}{l}\text { Captures the current } \\
\text { state of a device }\end{array}$ \\
\hline Content & Key-value string pairs & Arbitrary user-defined & Arbitrary user-defined \\
\hline Use cases & $\begin{array}{l}\text { Store a device's serial } \\
\text { number and } \\
\text { manufacturer } \\
\text { information as a key- } \\
\text { value pair }\end{array}$ & $\begin{array}{c}\text { Send a reboot } \\
\text { command to a device }\end{array}$ & $\begin{array}{l}\text { Retrieve a device's } \\
\text { health (such as the } \\
\text { frequency of crashes) }\end{array}$ \\
\hline \multirow{2}{*}{$\begin{array}{l}\text { Message direction } \\
\text { Frequency }\end{array}$} & Device-to-core & Core-to-device only & Device-to-core only \\
\hline & $\begin{array}{l}\text { No more often than } \\
\text { twice per process }\end{array}$ & Low & High \\
\hline
\end{tabular}

The process configuration data, as opposed to the metadata information would only be instantiated when a configuration procedure is started. This step needs only be monitored when a state has changed, and this will belong to the next type of information data.

Process state information resembles the actual data that includes the state of the process monitored. This information is used to represent the status of the process, and may include health data, crashes, and periodic updates to the system monitor that needs to be aware of it. This information is the most important in a monitor system framework, and because of that it will indeed marked with a higher priority that the rest of the monitor information.

\subsubsection{Description of the monitored process}

As there is a process to monitor, it would be of consideration to define how the process is defined, as an addition to the Section 5, where a process is firstly specified. This is a key part in the proposed framework, as is the basic element that the system will be have in charge, when services are added to the platform

The process engine is the former execution delegate in which the services depends to obtain the information in order to obtain proper results as an output of their execution. This engine relies in the transformational data produced by the YAML ('The Official YAML Web Site', n.d.) processor that generates the services decomposition module as a first approach to the execution step in the final devices. This process uses the metadata obtained in this phase to perform the functions associated at this level that are described as follows. This method is represented in Fig. 26. 
Management system is aware of the services requested by the user, as they are received by the user interface and filtered to obtain only the current possibilities to start the execution. This limitation would be imposed by the availability or the status of the other services executing at the same time. At this moment, a unique id is generated and assigned to the service (and added to its metadata). This id will be attached to the whole lifecycle of the services, as well the processes that it would produce.

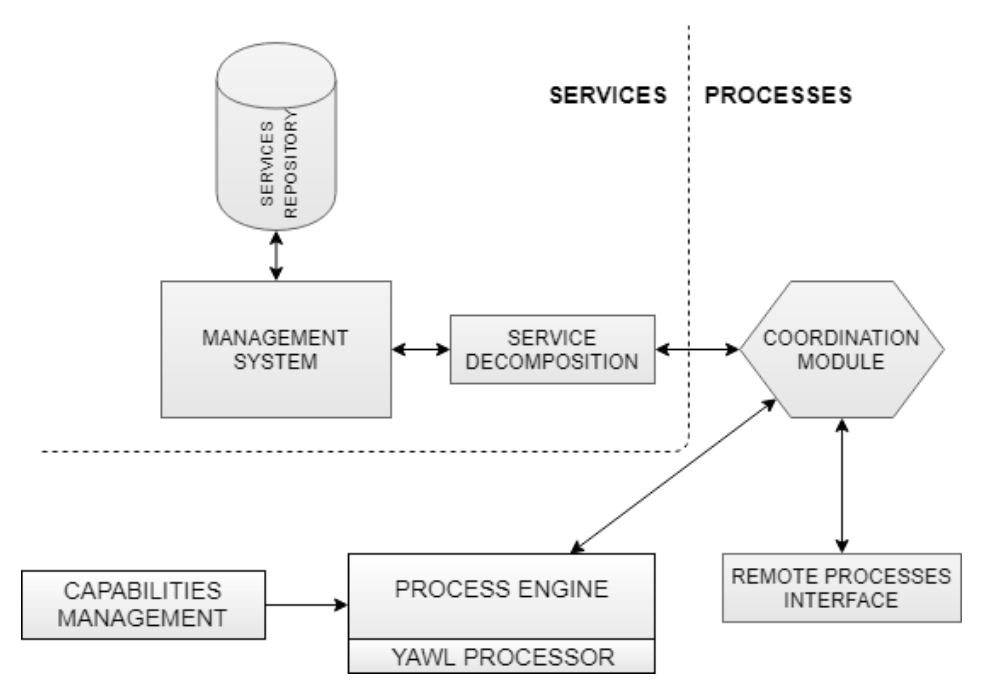

Fig. 26. Services to processes monitor modules

The service decomposition allows the division of the service requested in one or more processes that will be executed in the final devices. This component is the key component in the process workflow as it is in charge of define the process along with the metadata that in the next methods, the process engine executes. As Section 5 explains, the multisite requirements set the coordination module to be aware of the different executions taking place in the relevant locations. The decomposed output data also reflects that.

To integrate successfully the process creation with the proposed monitoring system, the service decomposition module needs to notify the monitor module and attach the respective unique ids for each created process. At this time, as the service is not replicated in every site, the monitor would expect the future incoming data and react at the first indication of successful or failure.

The coordination module in this phase would allocate in the monitor module the following information:

- Execution indicators: if any process is actually not executable, or if the process is duplicated, this information could be represented as a monitored information.

- Quality-of-Service: the representation of each process-desired factor is informed. Processes that not comply with this requirement would me marked to re-configure at the system management level. This may indicate that the system status has changed, or other critical events are happened. 
- Process cost of execution: only if there are several devices that could perform the same execution, all of them would be presented and monitored, but only the lowest cost associated would be the selected to execute the process.

\subsubsection{Monitoring the process lifecycle}

As stated, information related to the device status, captures the current state of the process, not the device information itself. Process can describe their status with a persistent and predefined set of data model that the device sent along carrying all the process information that it is executing (or loaded) in a certain time. The format used to this information is user-defined also, but in this proposal the YAML format is used, as it includes a greater possibility of description.

Related to the process lifecycle that is shown in Fig. 27 of a newly created service, once the system coordinator through its coordinator mirror engines identifies the final devices where the process has to be placed (launch state), an instruction to create the process is generated. This is the initial step to create a process in the device, and the process will gather the configuration parameters that are sent by the control plane though the common bus and the process is started. That information belongs to the process configuration classification.

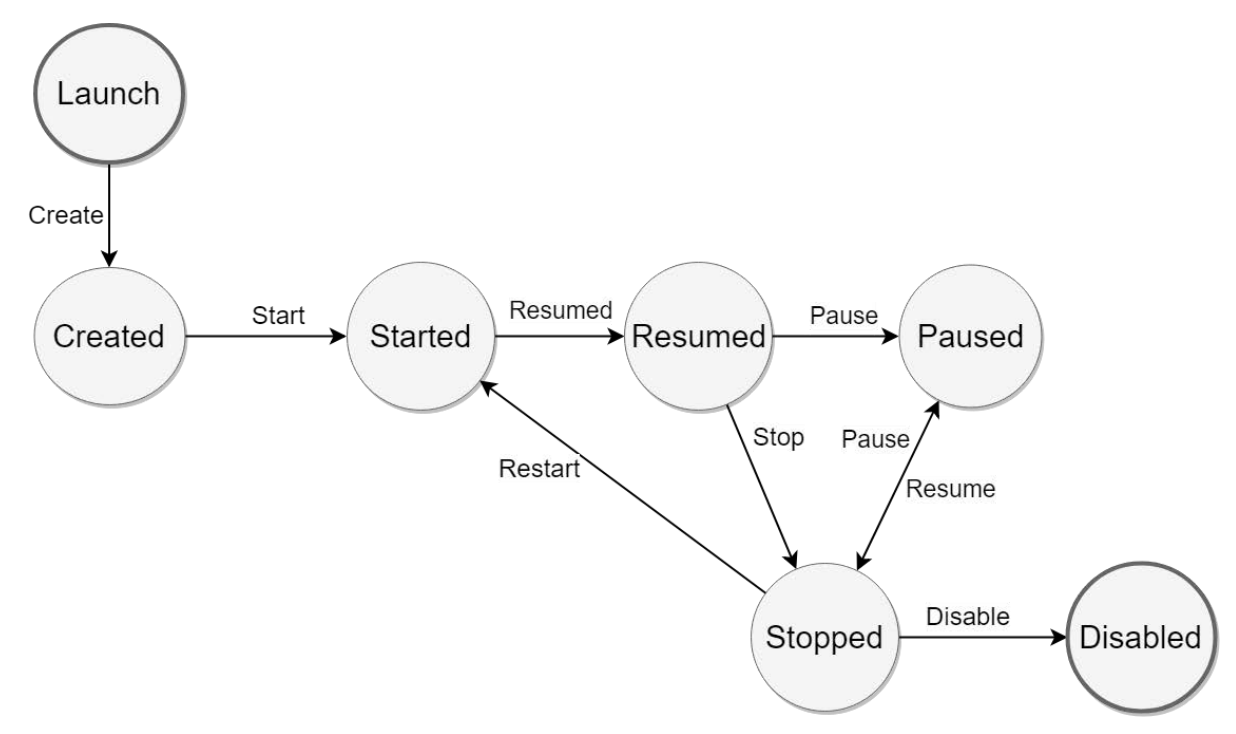

Fig. 27. Monitoring process lifecycle

As this is the primarily event, from that point, the monitor data flows in the upward direction to the system monitor. As the process is progressing on its load, the different states will provide more information and the state will be updated.

For example, a common process would start after the allocation of its resources on the device. This will lead to transmit the result of the device load to monitoring the status and this information need to be updated in the system monitor. The started status confirms the execution of the process and from that point a single path can be only follow to the resumed status. This, as the same as the created status is where the device must inform of their allocated resources to the system monitor, allowing to monitor the 
process lifecycle but with one difference. In this transition, the process has to inform its previous state, as the process can be already started but not paused or stopped previously.

Paused and stopped are states with minimal impact of the monitor data, as they cannot generate additional information until a resume or a restart command issue the continuation of the task, as noted in Table 3.

Table 3. Information and status relationships

\begin{tabular}{l|cccc}
\multicolumn{1}{c}{ Allocation } & Metadata & Configuration & Status \\
\hline Launch & $\mathrm{X}$ & & & $\mathrm{X}$ \\
Created & & $\mathrm{X}$ & $\mathrm{X}$ & \\
Started & & $\mathrm{X}$ & $\mathrm{X}$ \\
Resumed & & & $\mathrm{X}$ \\
Paused & & & $\mathrm{X}$ \\
Stopped & & $\mathrm{X}$ & $\mathrm{X}$ \\
Disabled & $\mathrm{X}$ & & &
\end{tabular}

It can be noted in the interaction table that the process lifecycle is constantly monitored by its relevant information. Distinguishing between the data model identified previously and the amount of data generated by the process evolution, can be sized with granular precision in order to avoid the overloading of the common bus. This will be tested and commented in Section 9 as a validation case will be implemented and measured.

\subsection{Distributed CPS monitoring, a resiliency focused approach}

In order to reduce the risks associated to the increment of slave nodes, it is necessary to consider certain objectives that our framework will succeed at them. At a first approach, getting the resiliency needed in the system to perform the required tasks will be useful to identify the most probable point of failures. At least, from the point of scalability issues they are as follows.

- Communications failure.

- Task failure.

- System component failure.

Those cases could happen unexpectedly at any time in the execution of a certain task, and for every case the fallback opportunity have to mitigate the possible drawbacks of the interruption. Inconsistencies in these cases are expected to happen and a simple architecture will provide simpler recovery methods than complex implementations.

With this in mind, I start to work on the most basic model of our approach. Considering only one coordinator slave and trying to implement only the necessary elements to perform the monitoring task, I propose to use two only modules that will be in charge of communicating the state of the working process between them. By 
communication them directly and removing extra elements, the data that can be redundant, is limited. This will allow to a simpler recovery when inconsistencies are found.

Using the secondary bus that is proposed in this PhD Thesis, Fig. 28 represents the use of the bus to communicate the two modules. Each module will be in charge of its monitor the status of the modules incorporated to the system. Nevertheless, the service status can be in a random state from its current operation and the monitor system is updated by the coordination slave, as it will handle the requests from the entities monitored in each site. The monitor system as it is, it is needed to be available in a highly percent of the time, so it can be reachable from the slaves.

The communications failure should be covered by the coordination slave, as it is capable of storage the current situation until the link is reestablished. This is managed by the on-site module, as there is no need in the system to send the state updates but trying to keep the consistency of the monitored information.

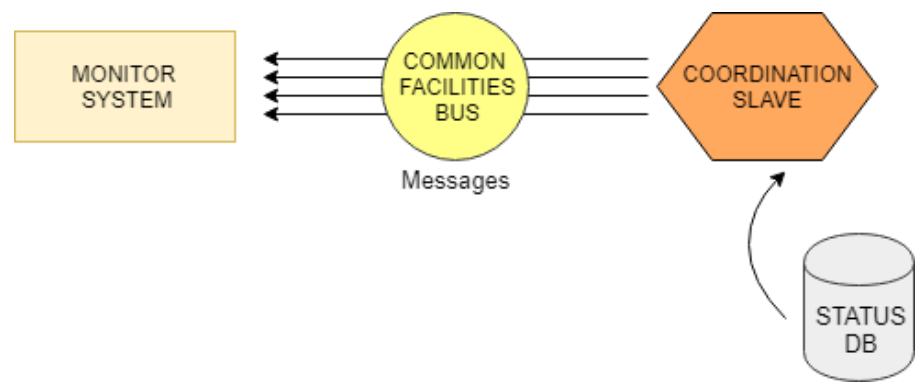

Fig. 28. One direction bus integration

In this basic system, the directions of the information messages are also defined. The problem when information is met in backwards propagation paths as stated before is forbidden by only letting the messages flow in one direction, from slaves to the monitor system. This manner, only the monitor can send commands to the slave, which will process it and generate the optional output as an information message and then dispatched to the system. Then, only one loop is permitted in the system, and thus, no additional loops are generated, avoiding redundant information and messages.

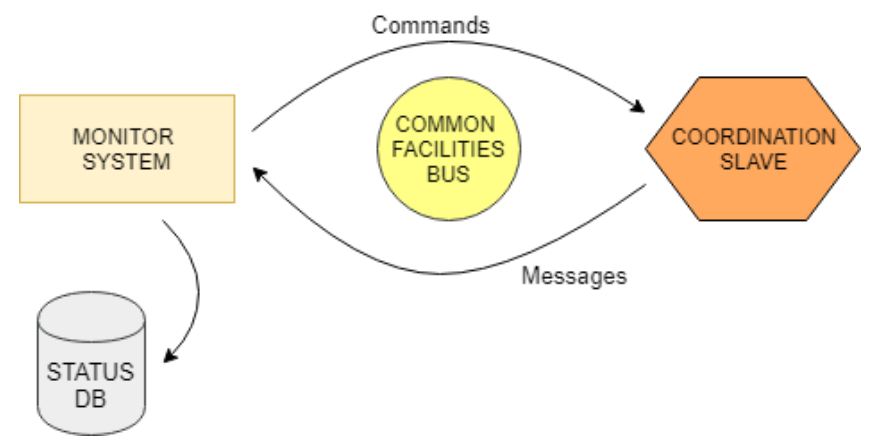

Fig. 29. Bi-directional bus integration

Allowing this command message to depart from the monitor system is defined based on the possibility of the monitor system to request specific information of the coordination slave. Information that is not usually sent in a regular basis will be available 
for the monitor system with and event driven basis, despite the time basis that the other messages could have. The bi-directional bus integration described is presented in Fig. 29.

\subsubsection{Benefits and drawbacks of the proposed approach}

Until now, a single coordination slave has been placed in the system, but designing a distributed framework requires scalable properties and implementation capabilities when working with more than one entity in the system. The simplicity of the singular proposal helps the reutilization of the architecture, in this case by replicating the schema in every site that the system needs to monitor. The more sites incorporated, it is clear that a more robust common bus is needed, although this requirement is fulfilled by the architecture provision of the complete system.

By the time that the global system is aware of additional sites, the system monitor will be in charge of declare the scope of the site coordination specifics, as it includes the entities of the modules to monitor and to stablish the relationship between the two colocation modules. This needs to be somehow resolved by the definition of a clear process declaration, that allows to declare a lineal lifecycle of all the operations and providing the necessary modules by the completion of the monitor chain in the system.

In this aspect, it is remarkable the adequate adaptation of the proposed monitoring framework to the different architectures analyzed in Section 3.2, where the presence of the common bus was discussed along the different implementations. The monitoring framework as it is proposed fits in the gap left by the key partners when searching for a complete model in this environment.

\subsection{A monitoring model for a distributed CPS}

As commented in Section 3, recent research showed up that the term monitoring is often found associate to the term real-time, as it resembles the objectives of the monitor process: getting updated information about elements in the system. When a monitor process is included in the system, a real-time constraint is imposed by the necessary failure awareness. In this context, this term allows to adapt the ideas and criteria used in the real-time computation to obtain a reliable monitored system, as described in the realtime data monitoring definition, from which I have extracted the different levels of requirements that a system can offer.

As shown in Table 4, the levels identified are divided in three, allowing adopting the more adequate for a certain system. This selection can be critical as the queues in the monitor data paths can be limiting.

\section{Table 4. Monitoring levels}

\begin{tabular}{l|c|c} 
Level & \multicolumn{1}{l}{ Description } & Primarily use \\
\hline Low & $\begin{array}{c}\text { Small data amount, Regular } \\
\text { intervals }\end{array}$ & High volume of messages \\
\cline { 2 - 3 } & &
\end{tabular}




\begin{tabular}{|c|c|c|}
\hline Medium & $\begin{array}{c}\text { Sampling data, Regular } \\
\text { intervals }\end{array}$ & Most objects \\
\hline High & All possible data & $\begin{array}{l}\text { Small data throughput, focus on } \\
\text { current information }\end{array}$ \\
\hline
\end{tabular}

The levels are selected based on the primary use that the system administrator requires in the system. This can help the understanding of the current state and provide useful information to diagnose a problem. As the framework is conceived to grow in a vertical and horizontal way, the ability of determine the queue saturation of the different elements is key and this is why the High level can be assumed to perform the sizing of my framework.

In this way, it can be clear that the main problem when designing a monitor framework can be the amount of granularity expected when choosing the High level of processing. As more entities included in the system, the quantity and the dispersion of the monitor data through the monitored paths is rapidly increasing.

In addition, monitoring process often aim to produce some feedback to the monitored execution. This can be consumed by an external component or entity, like a person in charge or to an automated feedback loop, which varies the work in progress at the same time. With this analysis, it is needed a formerly adaptation of the legacy monitor architectures to configure an updated model that will be scalable and failure-free proved.

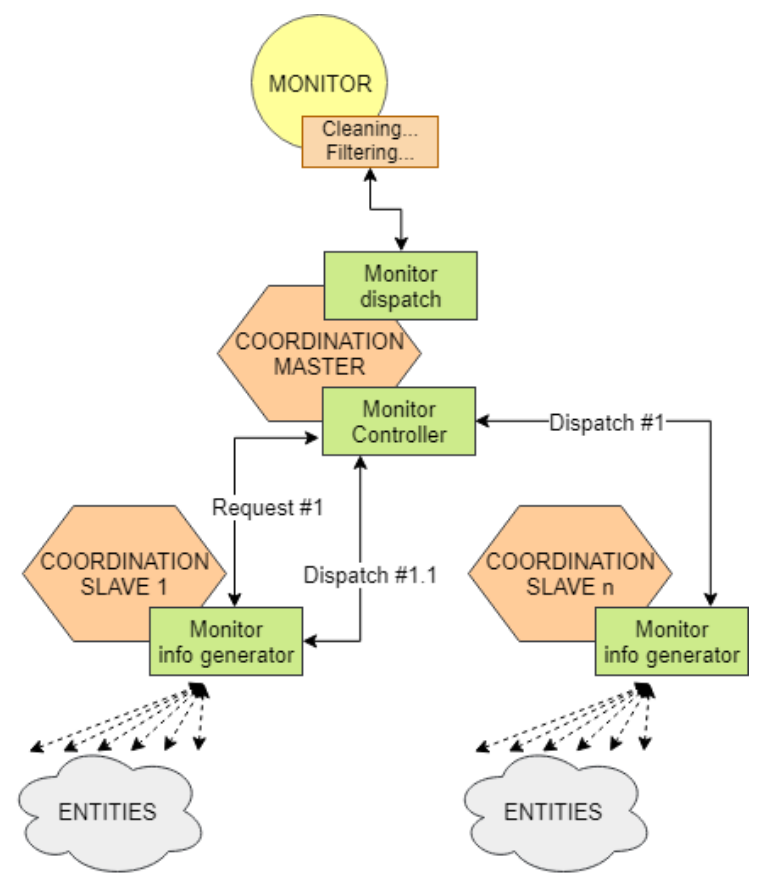

Fig. 30. Monitoring schema in a multi-site CPS

To explain the definition of the problem, a particularization of a cyber-physical system is made in order to granulize the different cases that a monitor system could be facing. As the proposed system is composed by several layers or sites, this desegregation 
would be implicit in the several modes identified, shown in Fig. 30. Nevertheless, this imposes some requirements to fulfill at the design level and the architecture has to take into account the high availability constraints to retain the high reliability required. Sites, as defined in section 5.1, are isolated entities that comprise a number of entities available to the global system and reachable by single or multiple gateways in a mater-slave coordination method. In order to monitor several sites in a distributed environment, a distributed framework would be the first choice when designing a platform, but some consideration have to take in mind then.

In relation to the information paths followed by the monitored data, several modules are reached by data until destination, where some processing could occur. In that data transmission, data triggers some processes at these modules which often result in more output data which is incorporated into the same path. As the processing occurred in this phase, the predicted data flow is assumed unidirectional, and with this objective the rest of the modules are scaled. This can be a challenge in a real scenario, where the actions created by processing the data can lead to unpredicted flows.

Those unpredicted data paths need to be controlled, as the data is passing through stablished routes to the destination, the possibility of additional data created in the opposite direction is relevant. This is where the contribution of this section is centered, providing a model to avoid those unwanted paths. The process execution phase of the monitoring elements is flail with the final objective of incorporate the unified route and avoiding auxiliary paths. When unpredicted paths appear in the system, as Fig. 31 presents, a more complicated result can occur; loops are often found in distributed systems when the distributed elements are not well coordinated.
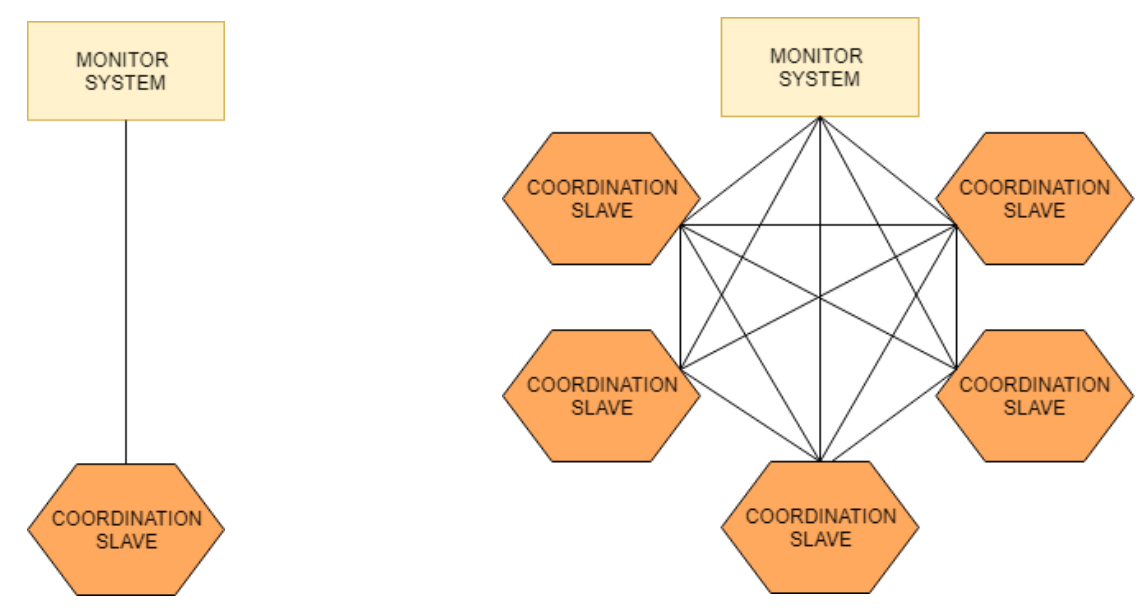

Fig. 31. Multi-site interconnection paths

Even with a distributed model like the presented in Section 5.1, the monitor system complexity can grow rapidly as more coordination slaves are added. The common facilities hub needs to be aware of each element and without a proper management model the status of every element will travel along the system and can be prone to data loss and inconsistencies with high probability. 


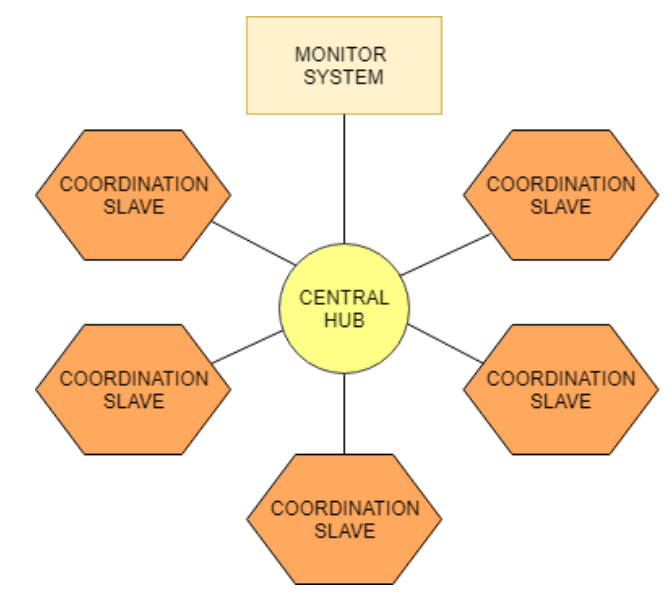

Fig. 32. Multi-site interconnection paths with central hub

Loops found in distributed systems can be simple communication paths where the triggers located in the processing modules produce backwards requests to the opposite direction that the data path is intended to travel. This is often necessary when additional information is needed from entities in a lower level. As the architecture is decentralized, this situation can occur more often, needing additional information from different off location sites and replicating data loops in every site that entities are located. To provide methods and an architecture focused on providing solutions to the loop problem I propose, in this Section 7, a novel execution framework focused on system monitoring that enforces the unidirectional path in each data transmission through the whole system. By separating the data processing elements found in the way to the final system from the additional information, it is avoided the need of trigger a new request at the same time in a concurrent model. By implementing this model, an asynchronous request is made, and appended to the system queue to be processed and thus converting the data transmission in a one at a time model, to be able to control the data paths by using a central hub to distribute the entire monitoring chain, as shown in Fig. 32.

In order to support the implementation of the method, it is necessary to stablish some definitions used by the proposed system related to the model of the service processing that the monitoring system is monitoring. This definition is not fixed to the explained system, but it is needed in order to identify some of the process lifecycle state that a general CPS process will have. Then, they will be used to model and define the elements of the presented architecture used to support the functionalities.

\subsection{Supporting the process execution monitoring}

The proposal of previous sections needs to be supported by an adequate architecture that satisfy the requirements imposed by the nature of a monitor system, and applies them in a seamless operation environment with all the functions embedded.

In order to support the process execution model into the Section 5, detailing the architecture proposal, it is essential to adapt and prepare the modules used in every step of the process. 
Having a common bus that is shared between all the co-located sites allows interacting with every component in a direct approach using none adaptors or interfaces that add complexity to the system. Besides avoiding process transformation in the path, the information passed by any entity on the system can be easily reached by other interdevices. This is the case with the entities involved in the multi-site location purposes.

Fig. 33 shows an abstraction of the monitor module interconnections, and the interfaces that are exposed to the platform. In the image, the management systems act as a merely information provider to the monitor module, as it needs to know the global status timely wise. Other connections allow incorporating the information stated in previous sections.

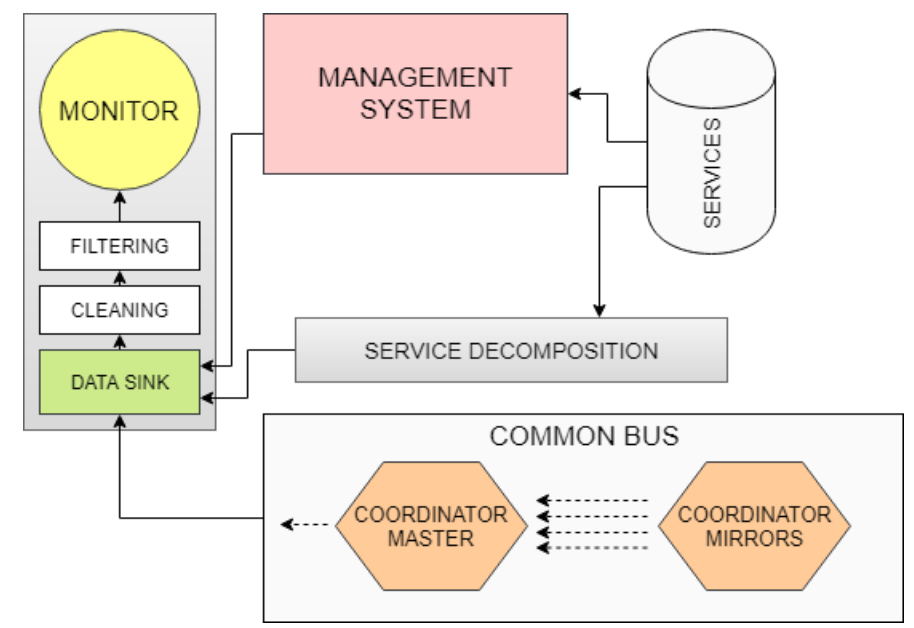

Fig. 33. Monitoring modules of the supported architecture

With the common bus, it is important to keep all the common communications in one place. In this case, the election of a proper communication protocol a significant task requires to know the volume of the monitored data. The requirements are imposed by the needs of a preferred direction of the data passing through the common bus. As stated before, the monitored data must travel in the upward direction due that, having the control of that, it benefits of noon loop operations in a multi-site location. The technology selected is the publish subscription protocol. Table 5 compares the key characteristics between the most used protocols, and states the right selection of a proper protocol.

Table 5. Comparison between Pub/Sub and Req/Res patterns

\begin{tabular}{lcc|}
\hline Best at & Pub/Sub pattern & Request-Response pattern \\
\hline $\begin{array}{l}\text { Require broker } \\
\text { Point of failure }\end{array}$ & $\begin{array}{c}\text { Multi-broker allows } \\
\text { to many connectivity }\end{array}$ & One to one connectivity \\
\hline $\begin{array}{l}\text { Additional } \\
\text { protocols }\end{array}$ & $\begin{array}{c}\text { Requires additional } \\
\text { protocol specific }\end{array}$ & Payload can be sent directly \\
\hline
\end{tabular}


As can be seen, publication and subscription pattern protocol fits to the requirement that the architecture imposes in a distributed environment. The focus on the many-tomany connectivity can be adapted to many-to-one directly and with the use of a multibroker configuration, the failures can be minimized. The only drawback is the need of specify a specific configuration in every entity. This can be even beneficial as it allows identifying the different entities by the use of hierarchical terms of publish topics.

Following with the supported methods in the architecture, process monitoring as explained in the Section 7.1.1, is sustained by the on-site modules that will reflect all the state transitions along with their status information through the same common bus that is being used by the coordinator mirrors.

\subsection{Summary}

In this section, I propose a method to monitor the requested executions in a serviceoriented CPS and to assure the validity of the executions status in a system composed by several site locations, as defined in Section 5. Managing a flexible CPS with ongoing processes in a multi-site environment, can be challenging and requires scalable solutions that allow the addressing of the all possible problems.

One of the requirements identified in Section 4, was $R E Q \# 7$, which refers to the capability of the system to be aware of the status of its components in a proactive manner. To know the execution state of the requests imposed by the application is a need for every system manager in order to provide feedback to the upper layer and to be able to recover from an unexpected situation in any case.

Section 7 presented the conditions that can be found in a running CPS. Then, and always focusing on monitor the services available on the system, a distributed CPS monitoring process is designed in order to address problems such as communication failures, task abortions or technology problems that can harm the monitoring process.

The monitoring of these components is a valuable feedback to the general system, and therefore, the application that uses the infrastructure will be improved with the information generated with this subsystem. 
7. MONITOR AND EXECUTION VALIDATION MODEL IN AN INTEROPERABLE MULTI-SITE CPS 


\section{Optimizing communication efficiency in constrained endpoint devices}

In previous sections, the multi-site CPS proposed was unaware of the final device implementation, although these devices are the final elements in which the system relies it's executions. In this section, I propose different scheduling models to optimize the battery usage at the hardware device level. Specifically, I propose the addition of different wake-up models to adapt the sleep routine in order to reduce the time in which the device is discoverable but trying to carry on the job without crucial delays. This is important in the constrained hardware devices present in a CPS, as often these devices are battery powered and implement low power components.

This section focuses on a series of methodologies that focus into enabling improved wireless communications to the heterogeneous battery powered devices in any CPS infrastructure; enabling low computer resource elements to wirelessly and battery powered communicate with other components. This is why battery performance is currently a key issue, influenced by multiple factors such as data transmission time and use of computational resources. There are some battery-saving solutions optimizing sleep periods and compressing transmitted data to reduce the power impact, but they are not optimized for its inclusion in CPS.

As low-energy devices are growing in the new paradigm of the wireless self-aware hardware systems, in the actual era of electronics, one of the main goals is to maximize the interoperability between hardware components and achieve a reasonable performance in the case of wireless devices. It can be distinguished two types of hardware devices related to the importance of them in a certain architecture.

A type of devices with high computing power are often performing main functions with complex hardware elements and need to be always on and plugged into a continuous power source in order to maintain the control of a system. These devices 
frequently work with wireless communications to control and manage other smaller devices with more limited resources and often only powered by a limited source of energy, such a battery. The available energy in these devices is a key element that it must be controlled exhaustively to minimize unnecessary current leaks and increase the runtime.

A second type of devices that are focus on low-energy transmission are low resource devices that try to achieve the communication draining as little energy as possible. Lowenergy communications depend almost entirely on the power and the duration of the transmission, and considering them, the consumption of the energy can be optimized in both ways. Transmission power is responsible for assuring the discoverability of the device in their range of operation and this is why the wireless devices need a sleep state to reduce and often disconnect the wireless interface. Otherwise, the amount of time that a transmission is being performed is easily connected to a software aspect, because it is directly related to the quantity of the data being transmitted through the link.

Both, sensors and endpoints, tend to be resource constrained devices in order to adapt the investment as much as possible to the users' needs. Hundreds, or even thousands, of resource constrained components are integrated into dense technological deployments, without power supply (devices usually employ batteries) or communication infrastructure but communicating through massive machine type communications or MMTC (Dawy, Saad, Ghosh, Andrews, \& Yaacoub, 2017) (solutions to automatically create communication links among devices). To make viable these new solutions, two conditions are usually considered: (i) sensors and endpoints must be interoperable with any server or Smart Home, and (ii) devices' lifetime must be large enough compared to the system lifetime. The first condition is met by including in every device many different configuration options; and the second one requires from managers to configure components to make the most efficient possible use of resources.

As MMTC are automatic technologies, the (dynamic) configuration process in sensors and endpoints must be automatic and fulfill the efficiency condition. Many different authors and works have addressed this problem in relation to sensor-server communications, but end point-server scenarios have been poorly studied. In particular, no automatic solution to obtain a dynamic and efficient configuration for endpoints has been reported. They are still, then, mostly manually configured during the deployment phase. However, current CPS endpoints devices present a very dynamic behavior, where the information characteristics (entropy, generation rate, etc.) can change dramatically in short periods.

The contribution of this section is, therefore, to describe an automatic and dynamic configuration algorithm for endpoint devices in CPS, increasing (as much as possible) the communication efficiency at every moment. This chapter proposes a definition for "communication efficiency" considering the most important costs associated to the operation of resource constrained endpoints, and the value of the obtained information. This mathematical model is employed to do a priori prediction about the server behavior 
and information characteristics, corrected with real measurements and Bayes theorem. Once it is detected the real sever pattern, the endpoint modifies its configuration to increase the communication efficiency, according to the proposed model. Basically, two relevant configuration parameters are controlled: the endpoint lifecycle and the information compression method.

\subsection{Global scenario. Efficiency definition}

In general, information endpoints, to be interoperable with different systems and technological solutions, must include several configuration options. These options, as said, are usually manually selected during the endpoint deployment. However, current CPS endpoint devices tend to present a very dynamic behavior with real-time characteristics, and permanent configurations do not enable efficient long-term communications. Changes in the type of content been sent to the endpoints, or in the information renewal rate, may turn an initially very efficient configuration, in a total waste of resources. The solution, then, is to allow a dynamic and smart configuration mechanism to be continuously running in the endpoints.

We are assuming endpoints have been already configured at network and service level, guarantying the connectivity and interoperability. Moreover, server may (or not) support a negotiation process to adapt the endpoints and server's behavior.

Fig. 34 shows the basic architecture for a communication link between a server and an endpoint in a Smart House.

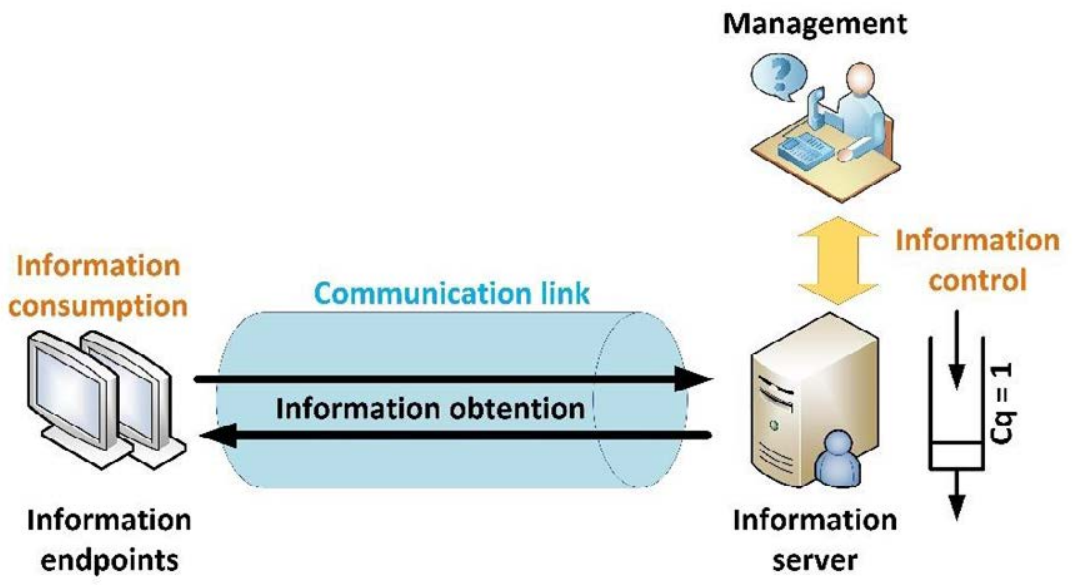

Fig. 34. Architecture for a communication endpoint-server link in CPS

As can be seen, content in managed by users, who can manually trigger an information actualization; or create automatic mechanisms through prosumer environments (B. Bordel, Iturrioz, Alcarria, \& Sanchez-Picot, 2018) to modify the system behavior and information characteristics according to a collection of rules or situations. These information control orders are the final cause of inefficiencies in fixed configurations, but they are external to any information system or solution, so they are not considered in this work. Thus, in our model, the information server embeds both, its own functionalities and behaviors inherit from managers. 
In this context, it is defined the efficiency in a communication link between an endpoint and an information server $\eta_{\text {com }}$ as the relation between the cost (value) of the consumed information $Q_{\text {infor }}$, and the total invested cost to recover and consume that information $Q_{\text {total }}(2)$.

$$
\eta_{\text {com }}=\frac{Q_{\text {info }}}{Q_{\text {total }}}
$$

As information blocks cannot be generated in a null time, for this analysis we are considering time as a discrete variable $n$, obtained from sampling the system situation each $T_{s}$ seconds. The value of $T_{s}$ is selected in such a way at least one information block can be generated during this period. The total (discrete) time employed to evaluate the system efficiency is $N_{s t u d y}$. If a longer operation time must be considered, it may be studied as a sequence of intervals

Now, we are formally analyzing all cost functions and values affecting the proposed efficiency rate, so global amounts are broken down in all elemental components. As different cost values represent the usage or different resources, to make amount comparable, all costs are ranging in the interval $[0,1]$ and are dimensionless. Thus, all cost calculation expressions include normalization parameters $\alpha_{i}$ to ensure those properties.

The cost (value) of the consumed information $Q_{\text {info }}$ is, in general, a function $C_{I}[\cdot]$ depending on the block generation time $n_{\text {gen }}$ and the time when the information is actually consumed (if so), $n_{\text {con }}$ (3). In general, as time passes, the value of consumed information decreases. All costs for all consumed blocks should be aggregated. Function $C_{I}[\cdot]$ is named as "cost function".

$$
Q_{\text {info }}=\frac{1}{\alpha_{\text {info }}}\left(\sum_{\forall \text { consumed block }} C_{I}\left[n_{\text {gen }}^{b_{i}}, n_{\text {con }}^{b_{i}}\right]\right)
$$

This amount, nevertheless, may be also written down as the balance between the (aggregated) original value of all generated information blocks, $Q_{\text {inf } o_{\text {total }}}$ and all lost value by those blocks, including non-consumed (lost in the server's queue, see Fig. 34) information blocks, $Q_{\text {info } o_{\text {lost }}}$ and the degradation suffered by consumed blocks because of time evolution, $Q_{\text {info }}$ deg $(4)$.

$$
Q_{\text {info }}=\frac{1}{\alpha_{\text {info }}}\left(Q_{\text {info }_{\text {total }}}-Q_{\text {info } o_{\text {lost }}}-Q_{\text {info } o_{\text {deg }}}\right)
$$

Cost of consumed information, although expressed as a balance between generated and lost cost (4), may be also calculated through cost functions (5). In this case, we are using a new cost function $C_{B}[\cdot]$, whose mathematical expression and meaning will be described in Section 8.2.1. 


$$
Q_{\text {info }}=\frac{1}{\alpha_{\text {info }}}\left(\sum_{\substack{\forall \text { produced block } \\ b_{i}}} C_{B}\left[b_{i}\right]-\sum_{\substack{\forall \text { lost block } \\ b_{i}}} C_{B}\left[b_{i}\right]-\sum_{\substack{\forall \text { consumed block } \\ b_{i}}}\left(C_{B}\left[b_{i}\right]-C_{I}\left[n_{\text {gen }}^{b_{i}}, n_{\text {con }}^{b_{i}}\right]\right)\right)
$$

In respect to the total invested $\operatorname{cost} Q_{\text {total }}$, considered in the efficiency definition (2), four basic components are identified in our model: the link management cost $Q_{\text {link }}$, the information obtention cost $Q_{\text {info obten }}$ and the information consumption cost, $Q_{\text {info }}$ consump (6).

$$
Q_{\text {total }}=\frac{1}{\alpha_{\text {total }}}\left(Q_{\text {link }}+Q_{\text {obten }}+Q_{\text {consump }}\right)
$$

The link management cost includes all effects and resource usage caused by the endpoint's lifecycle (7). It includes costs associated to wake up process, $Q_{\text {wake-up }}$, sleep process, $Q_{\text {sleep }}$, and stand-by process, $Q_{\text {stand-by }}$. Costs associated to link establishment and shutdown are not considered, as these processes are run during the system deployment (or disassembly) and, then, cannot be considered as an operation cost.

$$
Q_{\text {link }}=\frac{1}{\alpha_{\text {link }}}\left(Q_{\text {wake-up }}+Q_{\text {stand-by }}+Q_{\text {sleep }}\right)
$$

The cost of information obtaining, $Q_{\text {obten }}$, includes basically two processes: the query procedure to check for new information in the server, with a cost $Q_{\text {check, }}$, and the information recovery procedure, with a cost $Q_{\text {recov }}$. On the other hand, the information recovery cost is the aggregation of two different costs (8): the reception (or transmission) cost, $Q_{\text {recep }}$, and the decompression cost (if existing), $Q_{\text {decom }}$.

$$
Q_{\text {obten }}=\frac{1}{\alpha_{\text {obten }}}\left(Q_{\text {check }}+Q_{\text {recov }}\right)=\frac{1}{\alpha_{\text {obten }}}\left(Q_{\text {check }}+Q_{\text {recep }}+Q_{\text {decom }}\right)
$$

The last cost to be analyzed is the information consumption cost, $Q_{\text {consump }}$. This cost is directly related to the endpoints' functionalities and procedures they must perform to consume the received information (if any) or to process that no new information is available (for example, to display the received image or refresh the existing one). This cost will increment each time an endpoint looks for new information, according to function $\Sigma[\cdot]$. The cost to be added would be $Q_{\text {update }}$ or $Q_{n o-u p d a t e}$ depending on whether a new information block to be consumed is received or not (9).

$$
Q_{\text {consump }}=\Sigma\left[Q_{\text {update }}, Q_{\text {no-update }}\right]
$$

\subsection{Description of the proposed models}

\subsubsection{Information models}

In this work, information blocks (generated by the information server) are characterized by two main variables:

- Information blocks' lifetime: As said, current CPS are real-time solutions, and then information has a very short lifetime. In particular, in real-time applications (such 
as video streaming) an information block has value until a new and more recent block is produced. Then, the old block gets valueless. Here, we are considering the same model. As a consequence, information server (see Fig. 34) may be seen as a queue with unitary capacity $\left(C_{q}=1\right)$. Thus, any information block is stored waiting for being transmitted, until it is sent, or a new block is produced, when it is removed from the queue and replaced by the most modern block.

Contrary to scenarios where sensors send data to servers, where information has a long lifetime but loses value (cost) as time passes (Alcarria, Bordel, Robles, Martín, \& Manso-Callejo, 2018) due to physical processes continue evolving; in the proposed scenario (CPS with information endpoints), where endpoints receive information from servers, information keep the same value until it is totally replaced (valueless) by new information. This is an intuitive notion for images to be shown in displays, music, video, advertisements, etc.

In conclusion, for this analysis we are considering $Q_{\text {info }} o_{\text {deg }}=0$, as consumed information blocks are not degraded as time passes.

- Information Shannon's entropy, $H$ : For an information block $B$ codified with $k$-bit symbols, the Shannon entropy determines how many bits (in average) of these $k$ bits per symbol provide information. Considering its mathematical expression (10), this entropy parameter is maximum $(H=k$ bits) for totally random information blocks (equiprobable symbols); and minimum $(H=0)$ for blocks where only one symbol is employed.

$$
H(B)=-\sum_{s_{i} \in B} p\left(s_{i}\right) \log _{2} p\left(s_{i}\right) \quad \text { being } s_{i} k \text {-bit symbol }
$$

Using this measure (entropy) it is possible to define an objective cost function, to obtain the value (cost) of an information block $b_{i}$; i.e. we can define function $C_{B}[\cdot]$. First, considering the block entropy and $L$ as the block length (in $k$-bit symbols), we can calculate the amount of information, $I$, in any block (11). Then, as any cost must range between zero and the unit, but the information amount in a block varies between zero and infinity, the cost function must be an exponential law to agree with both ranges (12). In this cost function we introduce a free parameter, $\tau$, representing how fast or slow the block cost grows with the information amount in the block.

$$
\begin{gathered}
I\left(b_{i}\right)=H\left(b_{i}\right) \cdot L \\
C_{B}\left[b_{i}\right]=1-e^{-\frac{I\left(b_{i}\right)}{\tau}}=1-e^{-\frac{H\left(b_{i}\right) \cdot L}{\tau}}
\end{gathered}
$$

In order to obtain the final information cost $Q_{\text {info }}$, we must estimate the number of generated and lost blocks. To perform this calculation, we must model before the endpoints and server behavior. 


\subsubsection{Endpoint device and server models}

Basically, four different endpoints are considered in this work, describing the most common and employed behaviors for devices in information endpoints nowadays: (i) always-on model, (ii) fixed-period wake-up model, (iii) dynamic wake-up scheduling, and (iv) exponential evolution wake-up.

Always-on model is a trivial scenario, where endpoints are always enabled and available to receive new information from the server. Thus, endpoints are always powered, connectable and ready to react to any request. As endpoints in this model never get slept, it is easy to see that for these endpoints $Q_{\text {wake-up }}=Q_{\text {sleep }}=0$. Besides, because endpoints are never slept, they do not have to query the server for new information after being waking up. If any information is available, they just receive it. Then, $Q_{\text {check }}=0$.

Fixed-period wake-up model is also an elemental scenario, although it considers the waking up and sleep procedures. Basically, each $N_{\text {step }}$ time instants the endpoint wakes up, connects to the information server looking for new information blocks, performs the corresponding actions and gets slept another time. Fig. 35(a) shows a schematic chronogram describing this behavior. Being $N_{\text {sleep }}$ the time the endpoint is sleeping, this parameter has a constant value in this case (13).

$$
N_{\text {sleep }}=N_{\text {step }}
$$

The third model is more complicated. In the dynamic wake-up scheduling model, the information server is aware about the generation instant of the next information block, so it sends this datum together with the current information block to the endpoint. Then, the endpoint will sleep and wake up according to the received temporal scheduling. This model describes a wake-up scheduling that adapts the endpoint's sleep time to the block generation rate. In this way, the endpoint automatically adapts to different patterns if the system behavior changes. These wake-up scheduling, besides, aims to reduce inappropriate wake-up processes when there is no new information pending in the server. See Fig. 35(b).

Finally, the exponential evolution wake-up model describes a behavior which adapts the sleep period $N_{\text {sleep }}$ between checks for new blocks, exponentially increasing that time after each unsuccessful attempt to obtain new information from server, starting from a minimum value $N_{\text {sleep-min }}(14)$. See Fig. 35(c). The sleep time keeps constant after reaching a certain maximum value $N_{\text {sleep-max }}$. Besides, after a successful attempt to obtain new information from the server, the sleep time is fixed another time to its minimum value $N_{\text {sleep-min }}$.

$$
N_{\text {sleep }}=\left\{\begin{array}{c}
N_{\text {sleep-min }} \quad \text { if new block is recieved or } n=0 \\
2 \cdot N_{\text {sleep }} \quad \text { if no new block is recieved and } N_{\text {sleep }}<N_{\text {sleep-max }} \\
N_{\text {sleep-max }} \quad \text { if no new block is recieved and } N_{\text {sleep }} \geq N_{\text {sleep-max }}
\end{array}\right.
$$




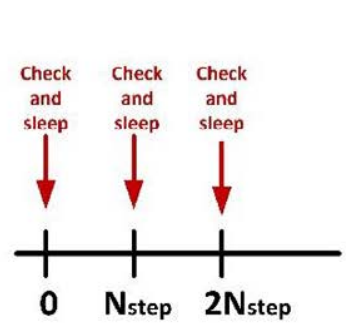

(a)

Fixed-period wake-up model

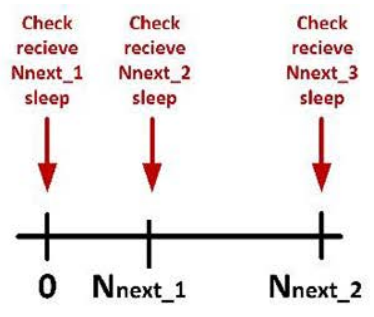

(b)

Dynamic wake-up scheduling model

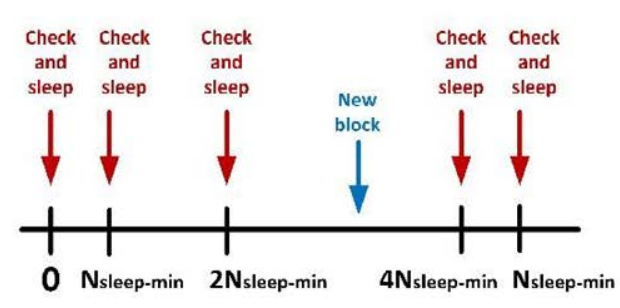

(c)

Exponential evolution wake-up model

Fig. 35. Lifecycle of endpoints according different behavior models

On the other hand, information server (which in our model embeds both managers' functionalities and its own) presents a behavior (in relation to information block creation) which may be modeled by a discrete stochastic process, $\delta[s, n]$. This process may take several different forms, representing each one a different information block creation pattern. However, in this paper, we are considering the three most common and relevant patterns: (i) predefined fixed pattern, (ii) stationary Bernoulli pattern and (iii) Poisson pattern.

In servers following a predefined fixed block creation pattern, the random variable $s$ is a Bernoulli variable where only two different states are considered (15). One value $(s=1)$ represents a situation where an information block is generated in the corresponding time instant. The other vale represents the opposite case. In this model, only one information block (as maximum) may be generated per time instant. As the block creation pattern is predefined, the probability of each state is known a priori for each time instant, and only take values in the $\mathbb{Z}^{2}=\{0,1\}$ set. In particular, it is known the set $\mathcal{N}_{\text {new-block }}(16)$ storing all time instants (15) for which $p_{\text {pre }}=p(s=1)=1$. At any other instant $p_{\text {pre }}=0$ (17).

$$
\begin{gathered}
s=\left[\begin{array}{l}
1 \\
0
\end{array}\right]=\left[\begin{array}{c}
\text { new block in sever } \\
\text { no new block in server }
\end{array}\right] \\
\mathcal{N}_{\text {new-block }}=\left[n_{1}^{\text {new }}, n_{2}^{\text {new }}, \ldots, n_{M}^{\text {new }}\right]
\end{gathered}
$$

On the other hand, severs presenting a stationary Bernoulli pattern have a similar behavior and model, but more general. In particular, the success probability $p_{b e r}=$ $p(s=1)$ is a real number in the interval $[0,1]$, the same value for all time instants (18).

$$
\mathcal{S}[s, n]=\left\{\begin{array}{c}
p_{\text {ber }}=\text { cte } \quad \forall n \text { per } \in[0,1] \\
q_{\text {ber }}=1-p_{\text {ber }}=\text { cte } \quad \forall n
\end{array}\right.
$$

Finally, servers following Poisson patterns are completely different. These severs are characterized by the generation of a certain mean number of information blocks $\lambda_{\text {poission }}$ each $N_{\text {study }}$ time units. In this case, besides, random variable $s$ takes values in $\mathbb{N}$, the set of the natural numbers (19). It represents the number of blocks generated in a certain time instant. 


$$
s \in \mathbb{N} \equiv\{0,1,2, \ldots\}
$$

Poisson servers are characterized by consecutive block generations distributed in time according to an exponential law. Statistic theory establishes that this behavior corresponds to a Poisson distribution with mean value $\frac{\lambda_{\text {poission }}}{N_{\text {study }}} \cdot n$ for ach time instant (20).

$$
\mathcal{S}[s, n]=P o i\left(\frac{\lambda_{\text {poission }}}{N_{\text {study }}} \cdot n\right)=\frac{1}{s !} e^{-\frac{\lambda_{\text {poission }}}{N_{\text {study }}} \cdot n} \cdot\left(\frac{\lambda_{\text {poission }}}{N_{\text {study }}} \cdot n\right)^{s}
$$

\subsection{Cost calculation procedures}

\subsubsection{Information cost calculation}

As said in Section 8.2, before obtaining the information cost, we must estimate the number of generated and lost blocks. These values, however, are random variables whose final expressions depends on the server's and endpoint's behaviors.

First, we are evaluating the number of generated information Blocks, $M_{B}$. In this case, as generated blocks are only dependent on the server behavior, the end point model does not have to be considered. Table 6 shows the obtained results for each server model.

\begin{tabular}{|c|c|}
\hline Server model & Probability distribution \\
\hline Predefined fixed pattern & $M_{B}=\operatorname{card}\left\{\mathcal{N}_{\text {new-block }}\right\}$ \\
\hline Stationary Bernoulli pattern & $p\left(M_{B}\right)=\left(\begin{array}{c}N_{\text {study }} \\
M_{B}\end{array}\right)\left(p_{\text {ber }}\right)^{M_{B}}\left(q_{\text {ber }}\right)^{N_{\text {study }}-M_{B}} \quad M_{B} \leq N_{\text {study }}$ \\
\hline Poisson pattern & $p\left(M_{B}\right)=\frac{1}{M_{B} !} e^{-\lambda_{\text {poission }}} \cdot\left(\lambda_{\text {poission }}\right)^{M_{B}}$ \\
\hline
\end{tabular}

Table 6. Total number of generated blocks. Random variables.

Servers behaving according to a predefined fixed pattern generate a fixed amount of information block, equal to the number of time instants when an information block is predefined to be created. Nevertheless, the other two server models generate a random number of blocks, so the corresponding probability distribution is proposed. For Bernoulli servers, the probability distribution may be calculated as a set of $M_{B}$ independent successful events, between $N_{\text {study }}$ trials. Then, a binomial distribution describes this distribution. For Poisson servers, the number of generated blocks is described by a Poisson distribution.

Now, on the other hand, we must calculate the number of lost blocks, $M_{L}$. Table 7 shows the corresponding values, or probability distributions (depending on the case) for all endpoint's and server's types. First, always-on endpoints receive information block just at the moment they are generated, so no block is never lost. However, fixed-period wake-up endpoints (as well as the other two possible endpoint's models) may be slept while several blocks are generated and lost. Then, all blocks above the unit generated 
while endpoints are slept, are lost (as only the newest information block has value, see Section 8.2). In general, therefore, $M_{L}$ information blocks will be lost if $M_{L}+1$ blocks are generated in $N_{\text {sleep }}$ seconds. For predefined fixed pattern servers, this amount is easily calculated considering the block creation patter. Moreover, a special case is the "synchronization situation" for fixed pattern servers. In this configuration, it is selected the sleep period $N_{\text {step }}$ to guarantee there are not lost block (21).

$$
N_{\text {step }}=\min \left\{n_{k-1}^{\text {new }}-n_{k}^{\text {new }}\right\} \quad k \in \mathbb{N}, n_{k}^{\text {new }}, n_{k-1}^{\text {new }} \in \mathcal{N}_{\text {new-block }}
$$

For other server models, being $M_{L}^{i}$ the number of lost blocks during the i-th sleep period of a device, it is easy to calculate the probability of each value of $M_{L}^{i}$ using the binominal or Poisson distribution (depending on the server type).

Besides, being $C_{\text {sleep }}$ the number of sleep periods in $N_{\text {study }}$ time units (22), and using a sequential decomposition process to analyze all possible lost block distributions in $C_{\text {sleep }}$ periods (and the corresponding probability) we can obtain the results shown in Table 7 for fixed-period wake-up endpoints.

$$
C_{\text {sleep }}=\frac{N_{\text {study }}}{N_{\text {step }}}
$$

Dynamic wake-up scheduling endpoints are more difficult to study, as their behavior is not predefined by any function. As said, in general, these end points adapt to the server behavior. Then, we are assuming (hereinafter) their behavior is as follows (previous works have proved these are the most efficient behaviors for each case (Sánchez-deRivera, Alcarria, Martín, \& Huecas, 2018)):

- For predefined fixed pattern servers dynamic wake-up scheduling endpoints are configured to follow the same server's pattern (so there are not lost blocks)

- For stationary Bernoulli servers, where all time instants have the same probability to generate an information block, it is selected (for this work) as the most profitable endpoint model a fixed-period wake-up model.

- Finally, for Poisson servers which generates information blocks according to an exponential law, it is selected (for this work) as the most profitable endpoint model an exponential evolution wake-up model.

Thus, considering precious assumptions, the number of lost blocks in each case may be obtained as explained for the corresponding endpoint model.

Finally, we must analyze exponential evolution wake-up endpoints. In predefined fixed pattern servers, it is possible to calculate the exact number of lost blocks as no random component is affecting the result. For Bernoulli servers, as well as for Poisson servers, the probability distribution may be also calculated using a sequential decomposition process where $C_{\text {exp }}$ different time (sleep) periods are considered. As time period have not a homogeneous duration, $C_{\text {exp }}$ is variable depending on each situation. Nevertheless, for clarity in the mathematical analysis (which in this case is pretty 
complex) we are considering in this initial work $C_{\text {exp }}$ is constant and its value it is fixed to the mean value among the possible variance interval (23).

$$
C_{\text {exp }} \in\left[1, \ldots, \frac{N_{\text {study }}}{N_{\text {min }}}\right] \Rightarrow E\left[C_{\text {exp }}\right]=\frac{N_{\text {study }}}{2 \cdot N_{\min }}=2^{r_{\text {exp }}} \cdot N_{\text {min }}
$$

Now, the loss probability depends on the real duration of each sleep period, which depends on the duration and number of generated blocks in the previous period. Then, conditional probabilities appear (to be aggregated and obtain the final absolute value). Besides, the sleep period gets increased only if no block is generated during the previous period; an event whose probability is controlled by the server natural probability distribution $p_{n}$, following a Binomial (or Poisson) distribution. Besides, all probabilities are parametric on the sleep period length, including the absolute loss probability $p_{l}$, employed to obtain the conditional probabilities. Finally, we must consider the probability of a unique information block to be generated at each time (sleep) period, $p_{\text {sucess }}$, which is calculated aggregating all possible cases according to probability laws (24).

$$
p(A)=\sum_{\forall B} p(A \mid B) \cdot p(B)
$$

The final expression deduction is a complex induction process, and mathematical expressions turn especially large if recursive loops are removed, but obtained results allow estimating in a very precise manner the lost block rate.

\begin{tabular}{|c|c|c|}
\hline $\begin{array}{l}\text { Server } \\
\text { model }\end{array}$ & $\begin{array}{l}\text { Endpoint } \\
\text { model }\end{array}$ & \\
\hline \multirow{4}{*}{$\begin{array}{l}\text { Predefined } \\
\text { fixed } \\
\text { pattern }\end{array}$} & Always-on & $M_{L}=0$ \\
\hline & $\begin{array}{l}\text { Fixed- } \\
\text { period } \\
\text { wake-up } \\
\text { model }\end{array}$ & $\begin{array}{c}M_{L}=\sum_{\substack{\forall r \in \mathbb{N} \\
r \cdot N_{\text {step }} \leq N_{\text {study }}}}\left(\operatorname{card}\left\{\left[n_{k}^{\text {new }}, k \in \mathbb{N},(r-1) \cdot N_{\text {step }} \leq n_{k}^{\text {new }}<r \cdot N_{\text {step }}\right]\right\}-1\right) \\
M_{L}^{\text {syn }}=0\end{array}$ \\
\hline & $\begin{array}{l}\text { Dynamic } \\
\text { wake-up } \\
\text { scheduling }\end{array}$ & $M_{L}=0$ \\
\hline & $\begin{array}{l}\text { Exponential } \\
\text { evolution } \\
\text { wake-up }\end{array}$ & $\begin{array}{c}\qquad M_{L}=\sum_{i} M_{L}^{i} \\
M_{L}^{i} \text { max. integer number such that } n_{k_{i}-1}^{\text {new }}-n_{k_{i}}^{\text {new }}>N_{\text {min }} \text { and } \\
n_{k_{i}-1}^{\text {new }}-n_{k_{i}+M_{L}^{i}}^{\text {new }}>2^{r} \cdot N_{\text {min }} \text { and } n_{k_{i}-1}^{\text {new }}-n_{k_{i}+M_{L}^{i}}^{\text {new }}<2^{r+1} \cdot N_{\min }\end{array}$ \\
\hline \multirow{3}{*}{$\begin{array}{l}\text { Stationary } \\
\text { Bernoulli } \\
\text { pattern }\end{array}$} & " Always-on & $M_{L}=0$ \\
\hline & $\begin{array}{l}\text { Fixed- } \\
\text { period } \\
\text { wake-up } \\
\text { model } \\
\end{array}$ & \multirow{2}{*}{$\begin{array}{c}p\left(M_{L}\right)=\sum_{r_{0}=0}^{M_{L}} \sum_{r_{1}=0}^{r_{0}} \ldots \sum_{r_{C_{\text {sleep }}=0}}^{r_{c_{\text {sleep }}-1}} p\left(M_{L}^{0}=M_{L}-r_{0}\right) \cdot\left(\prod_{i=1}^{r_{\text {sleep }}} p\left(M_{L}^{i}=r_{i-1}-r_{i}\right)\right) \cdot p\left(M_{L}^{c_{\text {sleep }}}=r_{C_{\text {sleep }}}\right) \\
p\left(M_{L}^{i}=M\right)=\left(\begin{array}{c}N_{\text {step }} \\
M+1\end{array}\right)\left(p_{\text {ber }}\right)^{M+1}\left(q_{\text {ber }}\right)^{N_{\text {step }}-M-1} \quad 1 \leq M \leq N_{\text {step }}-1\end{array}$} \\
\hline & $\begin{array}{l}\text { Dynamic } \\
\text { wake-up } \\
\text { scheduling }\end{array}$ & \\
\hline
\end{tabular}

Table 7. Total number of lost blocks. Random variables. 


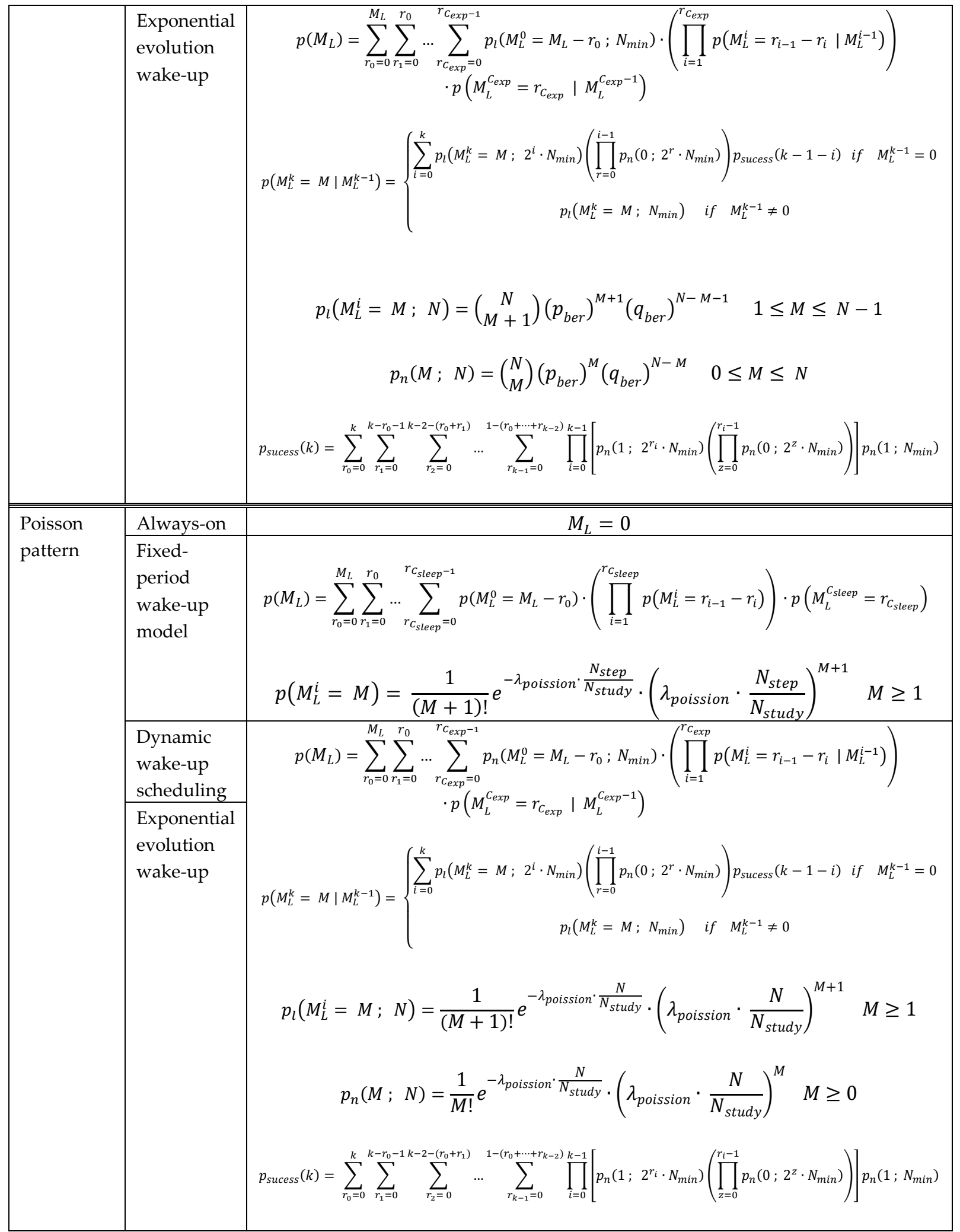

\subsubsection{Link management cost calculation}

The link management cost is totally caused by device lifecycle. Basically, costs under this name (cost of wake-up process $Q_{w a k e-u p}$, stand-by $Q_{s t a n d-b y}$, and sleep process $Q_{\text {sleep }}$ ) are associated to an energy consumption. All (mobile) endpoints have an 
independent and limited-capacity battery with an available electrical charge BAT, measured in ampere-hour. As, in this work, we are considering time as a discrete variable, it is necessary first to obtain the battery charge in ampere-discrete time units (25).

$$
B A T(A n)=\frac{B A T(A h)}{1 h} \cdot \frac{1 h}{T_{s}}
$$

Now, the cost of each charge unit should be variable and depend on the resting charge: as the battery is running out charge, the value of the resting energy grows up. Several different cost functions $C_{E}[\cdot]$ could be selected but all of them should fulfill the requirements described in Section 8.2.1 (cost ranges between zero and the unit). However, many different works (Borja Bordel, Miguel, Alcarria, \& Robles, 2018; Karlan \& Zinman, 2018) have proved the value of any resource goes up exponentially as it is sparser. Thus, we are also employing an exponential law as cost function (26). In this case it is a function with memory as previous consumptions, $B A T_{\text {con }}^{i}$, affect the resting energy cost. This function may be also written in a memory-less form if a new parameter $B A T_{\text {level }}$ is considered (27). In both cases, parameter $\tau$ (a real value) determines how fast the energy cost grows.

$$
\begin{gathered}
C_{E}=1-e^{-\frac{B A T}{\frac{B A T-\sum_{i} B A T_{\text {con }}^{i}}{\tau}-1}} \\
C_{E}\left[B A T_{\text {level }}\right]=1-e^{-\frac{B A T-B A T_{\text {level }}-1}{\tau}} \text { being } B A T_{\text {level }}=\sum_{i} B A T_{\text {con }}^{i}
\end{gathered}
$$

Previously presented cost function enables us to determine the cost of each charge unit, but some processes (such as stand-by) are running for several time units or consume higher current amounts. Then, the cost of a certain amount of charge units $E_{i}$ may be obtained integrating along the cost function (28).

$$
\begin{aligned}
& C_{E}\left[E_{i} ; B A T_{\text {level }}\right]=\int_{\overline{B A T-B A T} T_{\text {level }}}^{\frac{B A T T}{\left.B A A T \text { level }+E_{i}\right)}}\left(1-e^{-\frac{x-1}{\tau}}\right) d x=A+\frac{B A T}{B A T-\left(B A T_{\text {level }}+E_{i}\right)}+\tau \cdot e^{-\frac{\frac{B A T}{B A T-\left(B A T_{\text {level }}+E_{i}\right)}-1}{\tau}} \\
& A=-\frac{B A T}{B A T-B A T_{\text {level }}}-\tau \cdot e^{-\frac{\frac{B A T}{B A T-B A T_{\text {level }}}-1}{\tau}}
\end{aligned}
$$

Considering these mathematical expressions, we can now obtain the values for the three costs studied in this subsection. In respect to the wake-up process, we are considering it occurs in a one-time unit, consuming $I_{\text {wake-up }}$ amperes. Then, the unitary cost of each wake-up in any endpoint, $Q_{\text {wake-up }}^{\text {unitary }}$ may be easily calculated using the cost function (29). The same assumptions are applied to the sleep process, which consumes $I_{\text {sleep }}$ amperes; so the unitary cost of each sleep process $Q_{\text {sleep }}^{\text {unitary }}$ is also easily calculated (30). $B A T_{\text {level }}$ parameter must be fixed dynamically depending on the endpoint's situation.

$$
Q_{\text {wake-up }}^{\text {unitary }}\left(B A T_{\text {level }}\right)=C_{E}\left[I_{\text {wake-up }} \cdot 1 ; B A T_{\text {level }}\right]
$$




$$
Q_{\text {sleep }}^{\text {unitary }}\left(B A T_{\text {level }}\right)=C_{E}\left[I_{\text {sleep }} \cdot 1 ; B A T_{\text {level }}\right]
$$

While endpoints are slept, they do not consume energy, so no cost must be considered. However, while end points are on, a stand-by cost appears. While endpoints are in a stand-by state they consume $I_{\text {stand-by }}$ amperes. Considering the endpoint is on for a $N_{\text {stand-by }}$ length period, the unitary cost of a stand-by period $Q_{\text {stand-by }}^{\text {unitary }}$ may be calculated through the cost function (31). For endpoints which are on only for looking for updated, no stand-by cost is produced.

$$
Q_{\text {stand-by }}^{\text {unitary }}\left(N ; B A T_{\text {level }}\right)=C_{E}\left[I_{\text {stand-by }} \cdot N ; B A T_{\text {level }}\right]
$$

Then, for different endpoint's models, the global costs associated to wake-up and sleep processes and stand-by periods are different. Table 8 shows the calculation expressions for each case. We are assuming at $n=0$ batteries are totally charged.

For always-on endpoints, only the cost associated to the permanent stand-by state must be considered. For other endpoint's models no stand-by cost must be calculated. However, wake-up and sleep processes generate other costs to be obtained. In particular, the number of sleep periods for each model was calculated in Section 8.3.1. Therefore, it is enough to consider an aggregation of unitary costs, considering for each sleep period it is developed a sleep and a wake-up process. Besides, the battery charge decreases linearly according to consumed current for each process, so it is simple to obtain the battery level at each moment.

\begin{tabular}{|c|c|c|c|c|}
\hline \multicolumn{2}{|c|}{ Endpoint model } & \multicolumn{3}{|c|}{ Cost } \\
\hline & & $Q_{\text {wake-up }}$ & $Q_{\text {stand-by }}$ & $Q_{\text {sleep }}$ \\
\hline \multicolumn{2}{|l|}{ Always-on } & 0 & $Q_{\text {stand-by }}^{\text {unitary }}\left(N_{\text {study }} ; 0\right)$ & 0 \\
\hline \multicolumn{2}{|c|}{$\begin{array}{l}\text { Fixed-period wake-up } \\
\text { model }\end{array}$} & \multirow{2}{*}{$\sum_{i=1}^{c_{\text {sleep }}} Q_{\text {wake-up }}^{\text {unitary }}\left((i-1) I_{\text {wake-up }}\right)$} & 0 & \multirow{2}{*}{$\sum_{i=1}^{C_{\text {sleep }}} Q_{\text {sleep }}^{\text {unitary }}\left((i-1) I_{\text {sleep }}\right)$} \\
\hline \multirow{2}{*}{$\begin{array}{l}\text { Dynamic } \\
\text { wake-up } \\
\text { scheduling }\end{array}$} & $\begin{array}{l}\text { Bernoulli } \\
\text { server }\end{array}$ & & \multirow[b]{2}{*}{0} & \\
\hline & $\begin{array}{l}\text { Poisson } \\
\text { server }\end{array}$ & \multirow{2}{*}{$\sum_{i=1}^{C_{\text {exp }}} Q_{\text {wake-up }}^{\text {unitary }}\left((i-1) I_{\text {wake-up }}\right)$} & & \multirow{2}{*}{$\sum_{i=1}^{c_{\text {exp }}} Q_{\text {sleep }}^{\text {unitary }}\left((i-1) I_{\text {sleep }}\right)$} \\
\hline $\begin{array}{l}\text { Exponential } \\
\text { wake-up }\end{array}$ & evolution & & 0 & \\
\hline
\end{tabular}

Table 8. Link management costs for different endpoint's models

\subsubsection{Information obtention cost calculation}

Once evaluated the loss probability and the link management cost, we must address the cost associated to information obtention (considering the server has a new information block to be sent). This cost, $Q_{\text {obten }}$, is the composition of two partial costs: query process cost, $Q_{\text {check }}$, and the recovery process cost, $Q_{\text {recov }}$.

The query process cost refers the usage of hardware resources such as communications modules. Each query has an unitary cost, $Q_{\text {check }}^{\text {unitary }}$. As the elements 
affecting this cost are not consumable, we are assuming it has a constant value selected according to the endpoint implementation (we are not addressing hardware details in this work). Besides, as always-on endpoints are never slept, they never query the server for new blocks: if any block is available, the server just sends it. Table 9 shows the cost calculation for the different endpoint models, considering the number of sleep periods (a query process is performed after wake-up).

Table 9. Query process cost for different endpoint's models

\begin{tabular}{|l|c|c|}
\hline \multicolumn{2}{|c|}{ Endpoint model } & Cost $\boldsymbol{Q}_{\text {check }}$ \\
\hline \multicolumn{2}{|l|}{ Always-on } & 0 \\
\hline \multicolumn{2}{|l|}{ Fixed-period wake-up model } & $C_{\text {sleep }} \cdot Q_{\text {check }}^{\text {unitary }}$ \\
\hline $\begin{array}{l}\text { Dynamic wake-up } \\
\text { scheduling }\end{array}$ & Bernoulli server & \\
\cline { 2 - 2 } & Poisson server & $C_{\text {exp }} \cdot Q_{\text {check }}^{\text {unitary }}$ \\
\hline \multicolumn{2}{|l|}{ Exponential evolution wake-up } & \\
\hline
\end{tabular}

Now, the information recovery cost is not related to endpoints' characteristics, but to communication system's configuration. This cost is the aggregation of two amounts: the information reception cost, $Q_{\text {recep }}$, and the decompression cost, $Q_{\text {decom }}$.

In respect to the information reception cost, we are assuming a unitary cost describing the cost of receiving a bit, $Q_{\text {recep }}^{\text {unitary }}$. This cost includes spectrum reservation, reception buffers, etc. Then, for a received information block with a length of $L$ h-bit symbols (see Section 8.2.1), the reception cost may be easily obtained (32).

$$
Q_{\text {recep }}=L \cdot h \cdot Q_{\text {recep }}^{\text {unitary }}
$$

Now, to analyze the decompression cost we must consider different compression methods. In this work we are taken into account three different algorithms: (i) Runlength encoding (RLE), (ii) Huffman-Qopt method (where $Q$ is a parameter indicating the number of symbols to be employed in compressed blocks) and (iii) no compression (raw transmission). All decompression methods are modeled as the following sequence: a compressed symbol is read, and then the equivalent decompressed symbol is written. This sequence is repeated until the entire message is read. Mathematically, then, the decompression cost may be modeled as an algebraic operation (33).

$$
Q_{\text {decom }}=\left(h \cdot Q_{\text {read }}^{\text {unitary }}+k \cdot Q_{\text {write }}^{\text {unitary }} \cdot L_{\text {symbol }}^{\text {decompred }}\right) \cdot L_{\text {compressed }}+Q_{R A M}
$$

In this expression, different unitary costs are considered referring the consumed processing time: the unitary cost of reading a bit $Q_{\text {read }}^{\text {unitary }}$, and the unitary cost of writing a bit, $Q_{\text {write }}^{\text {unitary }}$. In general, compressed symbol are h-bit symbols, where $k \neq h$. Besides, $L_{\text {compressed }}$ refers the compressed information block length in h-bit symbols; and $L_{\text {symbol }}^{\text {decompred }}$ refers the amount of $\mathrm{k}$-bit symbol obtained from a compressed h-bit symbol. Finally, $Q_{R A M}$ is the cost of all transitory data structures (in volatile memory) needed to perform the decompression process. Considering an endpoint has a RAM memory with capacity $R A M$, the cost may be calculated using a cost function $C_{M}[\cdot]$ (34) 
isomorphic to the one described for energy cost (26) and considering $R A M_{\text {con }}$ the consumed memory amount.

$$
C_{M}=1-e^{-\frac{R A M}{R A M-R A M_{c o n}-1}}
$$

For raw transmission the decompression cost is zero, $Q_{\text {decom }}=0$, as no compression method is employed. Besides, RLE does not require any transitory data structure, so $Q_{R A M}=0$. Table 10 shows the relation between the compressed and raw length of an information block $b_{i}$, depending on its entropy. These expressions may be deducted considering how the different algorithms work (Sánchez-de-Rivera et al., 2018). For Huffman Q-optimum algorithm $H_{\min }^{Q+1}\left(b_{i}\right)$ represents the minimum entropy for a block $b_{i}$, calculated among all possible dictionaries with $Q+1$ symbols.

Table 10. Query process cost for different endpoint's models

\begin{tabular}{|c|c|c|c|}
\hline $\begin{array}{l}\text { Compression } \\
\text { method }\end{array}$ & $\begin{array}{l}\text { Message } \\
\text { length (raw) } \\
\text { k-bit } \\
\text { symbols }\end{array}$ & $\begin{array}{l}\text { Message length (compressed) } \\
\text { h-bit symbol } \\
\qquad L_{\text {compressed }}\end{array}$ & $\begin{array}{l}\text { Number of k-bit symbols per } \\
\text { compressed symbol } \\
L_{\text {symbol }}^{\text {decompred }}\end{array}$ \\
\hline Raw & $L$ & $L \quad(h=k)$ & 1 \\
\hline RLE & $L$ & $\frac{L}{\left(2^{h-k}-1\right)-\left(2^{h-k}-2\right) \cdot \frac{H\left(b_{i}\right)}{k}}$ & $\left(2^{h-k}-1\right)-\left(2^{h-k}-2\right) \cdot \frac{H\left(b_{i}\right)}{k}$ \\
\hline $\begin{array}{l}\text { Huffman- } \\
\text { Qopt }\end{array}$ & $L$ & $\begin{array}{c}\frac{L}{h} \cdot \frac{k}{k_{Q}} \cdot H_{\min }^{Q+1}\left(b_{i}\right) \\
k_{Q}=\left\lceil\log _{2}(Q+1)\right\rceil \\
H_{\text {min }}^{Q+1}\left(b_{i}\right)=\min _{\text {Dictionary } Q+1}\left(H\left(b_{i}\right)\right)\end{array}$ & $\left(\frac{h-k_{Q}}{k}\right) H\left(b_{i}\right)+k_{Q}$ \\
\hline
\end{tabular}

In these expressions, randomness is embedded by block's entropy, which is at the end a stochastic term.

\subsubsection{Information consumption cost calculation}

Finally, once an information block is received by an endpoint (or not), it must be consumed, and operations related to actualization, decision and device management must be taken. These operations have a cost $Q_{\text {consump }}$. Basically, two different costs may appear each time an endpoint looks for new information blocks:

- If server has in the queue any new information block, the endpoint (after obtaining it) must consume it (for example, display the retrieved image). Later, memory must be cleaned, timers programmed, etc. All this process has a cost Qupdate . 
- On the other hand, if no new block is available, the endpoint only must refresh the existing information, program timers, etc. All these operations have a cost, $Q_{\text {no-update }}$, which is usually is lower than the cost including the new information processing. In always-on endpoints, this cost is not applicable as they develop an alternative stand-by cycle.

For server following a deterministic behavior, then, $Q_{\text {consump }}$ may be directly calculated. For servers with a random behavior (Poisson and Bernoulli servers), a probability distribution is obtained. Table 11 shows the obtained expression for each case.

First, we must consider that, contrary to other costs, information consumption cost $Q_{\text {consump }}$ cannot take any real or integer value. Only values decomposable as a combination of $Q_{\text {update }}$ and $Q_{n o-u p d a t e}$ costs are possible values (35). Additional limits could be applied for certain combinations of server and endpoint model.

$$
Q_{\text {consump }}=M_{1} \cdot Q_{\text {update }}+M_{2} \cdot Q_{\text {no-update }} \text { being } M_{1}, M_{2} \in \mathbb{N}
$$

Now, for always-on endpoints, it is easy to obtain the value (or probability distribution) of the global cost, as only an amount of $Q_{\text {update }}$ units must be added each time the server generates a new information block. Equally, for fixed-period wake-up endpoints, it is only needed to evaluate the probability of generating, at least, one information block in each sleep period. That may be done using directly the Binomial or Poisson distribution, depending on the server type (Bernoulli or Poisson).

As said in Section 8.2.2, dynamic wake-up scheduling endpoints follow other models depending on the server type, so no new calculation are required.

Finally, exponential evolution wake-up endpoints require larger and more complex discussions. When employed with predefined fixed pattern servers, a deterministic calculation may be done. However, when employed Poisson or Bernoulli servers it is necessary to consider the Bayes laws (23) to obtain the global probability distribution. This mathematical development has been also employed in other subsections, although in this case a binary probability $p_{b}$ is defined, to calculate the natural probability of a Bernoulli (and Poisson) distribution to generate any amount of information blocks or (in the opposite case) not generate any block.

Table 11. Information consumption cost. Random variables.

\begin{tabular}{|c|c|c|}
\hline $\begin{array}{l}\text { Server } \\
\text { model }\end{array}$ & $\begin{array}{l}\text { Endpoint } \\
\text { model }\end{array}$ & \\
\hline \multirow{3}{*}{$\begin{array}{l}\text { Predefined } \\
\text { fixed } \\
\text { pattern }\end{array}$} & Always-on & $Q_{\text {consump }}=\operatorname{card}\left\{\mathcal{N}_{\text {new-block }}\right\} \cdot Q_{\text {update }}$ \\
\hline & \multirow[t]{2}{*}{$\begin{array}{l}\text { Fixed- } \\
\text { period } \\
\text { wake-up } \\
\text { model }\end{array}$} & $Q_{\text {consump }}=\sum_{i=1}^{C_{\text {sleep }}} Q_{\text {step }}(i)$ \\
\hline & & $Q_{\text {step }}(i)=\left\{\begin{array}{ccc}Q_{\text {update }} & \text { if } \exists n_{k}^{\text {new }} \in \mathcal{N}_{\text {new-block }} & \vdots \\
& Q_{\text {no-update }} & \text { else }\end{array}\right.$ \\
\hline
\end{tabular}




\begin{tabular}{|c|c|c|}
\hline & $\begin{array}{l}\text { Dynamic } \\
\text { wake-up } \\
\text { scheduling }\end{array}$ & $Q_{\text {consump }}=\operatorname{card}\left\{\mathcal{N}_{\text {new-block }}\right\} \cdot Q_{\text {update }}$ \\
\hline & $\begin{array}{l}\text { Exponential } \\
\text { evolution } \\
\text { wake-up }\end{array}$ & $\begin{array}{c}Q_{\text {consump }}=\sum_{\mathcal{N}_{\text {new-block }}} Q_{\text {update }}+M \cdot Q_{\text {no-update }} \\
M \text { integer number such that } n_{k_{i}-1}^{\text {new }}-n_{k_{i}}^{\text {new }}>N_{\text {min }} \text { and } \\
n_{k_{i}-1}^{\text {new }}-n_{k_{i}+r}^{\text {new }}>2^{M-1} \cdot N_{\text {min }} \text { and } n_{k_{i}-1}^{\text {new }}-n_{k_{i}+r}^{\text {new }}<2^{M} \cdot N_{\text {min }}\end{array}$ \\
\hline \multirow{3}{*}{$\begin{array}{l}\text { Stationary } \\
\text { Bernoulli } \\
\text { pattern }\end{array}$} & Always-on & $p\left(Q_{\text {consump }}=M \cdot Q_{\text {update }}\right)=\left(\begin{array}{c}N_{\text {study }} \\
M\end{array}\right)\left(p_{\text {ber }}\right)^{M}\left(q_{\text {ber }}\right)^{N_{\text {study }}-M}$ \\
\hline & $\begin{array}{l}\text { Fixed- } \\
\text { period } \\
\text { wake-up } \\
\text { model } \\
\text { Dynamic } \\
\text { wake-up } \\
\text { scheduling }\end{array}$ & $\begin{array}{c}p\left(Q_{\text {consump }}=M_{1} \cdot Q_{\text {update }}+M_{2} \cdot Q_{\text {no-update }}\right)=\left(\begin{array}{c}C_{\text {sleep }} \\
M_{1}\end{array}\right)\left(p_{\text {sucess }}\right)^{M_{1}}\left(1-p_{\text {sucess }}\right)^{C_{\text {sleep }}-M_{1}} \\
p_{\text {sucess }}=\sum_{k=1}^{N_{\text {step }}}\left(\begin{array}{c}N_{\text {step }} \\
k\end{array}\right)\left(p_{\text {ber }}\right)^{k}\left(q_{\text {ber }}\right)^{N_{\text {step }}-k} \\
\text { being } M_{1}, M_{2} \in \mathbb{N} \quad M_{1}+M_{2}=C_{\text {sleep }}\end{array}$ \\
\hline & $\begin{array}{l}\text { Exponential } \\
\text { evolution } \\
\text { wake-up }\end{array}$ & 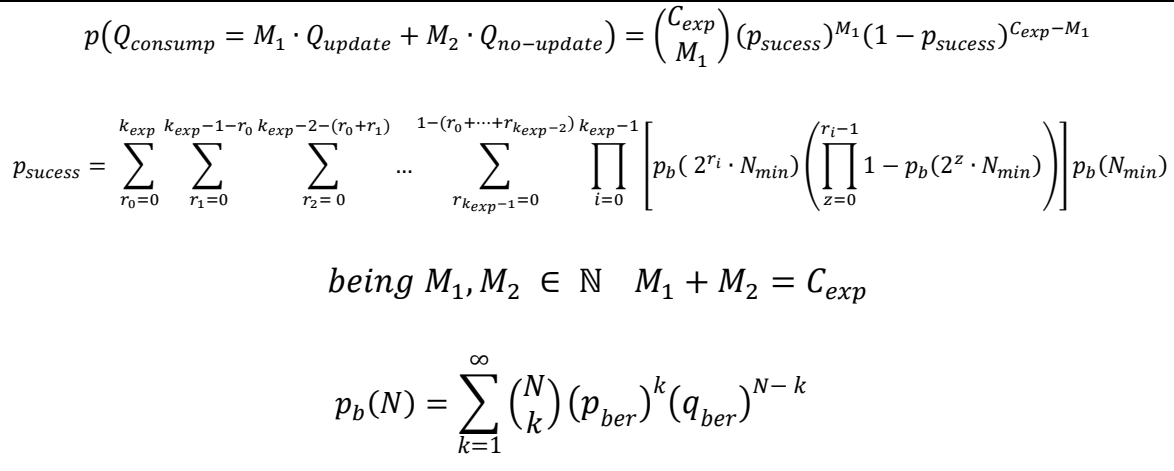 \\
\hline \multirow[t]{3}{*}{$\begin{array}{l}\text { Poisson } \\
\text { pattern }\end{array}$} & Always-on & $\begin{array}{c}p\left(Q_{\text {consump }}=M \cdot Q_{\text {update }}\right)=\left(\begin{array}{c}N_{\text {study }} \\
M\end{array}\right)\left(p_{\text {sucess }}\right)^{M}\left(1-p_{\text {sucess }}\right)^{N_{\text {study }}-M} \\
p_{\text {sucess }}=\sum_{k=1}^{\infty} \frac{1}{k !} e^{-\frac{\lambda_{\text {poission }}}{N_{\text {study }}}} \cdot\left(\frac{\lambda_{\text {poission }}}{N_{\text {study }}}\right)^{k}\end{array}$ \\
\hline & $\begin{array}{l}\text { Fixed- } \\
\text { period } \\
\text { wake-up } \\
\text { model }\end{array}$ & $\begin{array}{c}p\left(Q_{\text {consump }}=M_{1} \cdot Q_{\text {update }}+M_{2} \cdot Q_{\text {no-update }}\right)=\left(\begin{array}{c}C_{\text {sleep }} \\
M_{1}\end{array}\right)\left(p_{\text {sucess }}\right)^{M_{1}}\left(1-p_{\text {sucess }}\right)^{C_{\text {sleep }}-M_{1}} \\
p_{\text {sucess }}=\sum_{k=1}^{\infty} \frac{1}{k !} e^{-\frac{\lambda_{\text {poission }}}{N_{\text {study }}} N_{\text {step }}} \cdot\left(\frac{\lambda_{\text {poission }}}{N_{\text {study }}} N_{\text {step }}\right)^{k} \\
\text { being } M_{1}, M_{2} \in \mathbb{N} \quad M_{1}+M_{2}=C_{\text {sleep }}\end{array}$ \\
\hline & $\begin{array}{l}\text { Dynamic } \\
\text { wake-up } \\
\text { scheduling } \\
\text { Exponential } \\
\text { evolution } \\
\text { wake-up }\end{array}$ & $\begin{array}{c}p\left(Q_{\text {consump }}=M_{1} \cdot Q_{\text {update }}+M_{2} \cdot Q_{\text {no-update }}\right)=\left(\begin{array}{c}C_{\text {exp }} \\
M_{1}\end{array}\right)\left(p_{\text {sucess }}\right)^{M_{1}}\left(1-p_{\text {sucess }}\right)^{C_{\text {exp }}-M_{1}} \\
p_{\text {sucess }}=\sum_{r_{0}=0}^{k_{\text {exp }}} \sum_{r_{1}=0}^{k_{\text {exp }}-1-r_{0} k_{\text {exp }}-2-\left(r_{0}+r_{1}\right)} \sum_{r_{2}=0}^{1-\left(r_{0}+\cdots+r_{\left.k_{\text {exp }}-2\right)}\right.} \cdots \sum_{r_{\text {exp }}=0} \prod_{i=0}^{k_{\text {exp }}-1}\left[p_{b}\left(2^{\left.r_{i} \cdot N_{\text {min }}\right)}\left(\prod_{z=0}^{r_{i}-1} 1-p_{b}\left(2^{z} \cdot N_{\text {min }}\right)\right)\right] p_{b}\left(N_{\text {min }}\right)\right. \\
\text { being } M_{1}, M_{2} \in \mathbb{N} \quad M_{1}+M_{2}=C_{\text {exp }}\end{array}$ \\
\hline
\end{tabular}




$$
p_{b}(N)=\sum_{k=1}^{\infty} \frac{1}{k !} e^{-\frac{\lambda_{\text {poission }}}{N_{\text {study }}} \cdot N} \cdot\left(\frac{\lambda_{\text {poission }}}{N_{\text {study }}} \cdot N\right)^{k}
$$

The last problem we must address in this subsection if the calculation of partial costs $Q_{\text {update }}$ and $Q_{\text {no-update }}$. Basically, these costs refer the usage of hardware components such as microprocessors, RAM memory, displays, etc. A combination of different cost functions depending on the endpoint implementation should be employed. In this initial work, as we are not addressing implementation particularities, we are assuming these two partial costs have a predefined value representing the resource consumption according to the endpoint hardware.

\subsection{Proposed algorithm for optimizing communication efficiency}

In order to propose an optimization algorithm in a reasoned manner, we are firstly analyzing the behavior of the communication efficiency according to some relevant parameters using the previously described models. A multi-dimensional analysis would be required to consider all possible cases and situations, but this approach cannot be implemented in practice for more than two parameters. Therefore, we are focusing our analysis on those parameters the endpoints may change or negotiate: the endpoint's lifecycle and the compression method.

Fig. 36 shows the evolution of the mean communication efficiency for the different endpoint's lifecycles, considering one sever following a predefined pattern. Results are evaluated for different values of $\operatorname{card}\left\{\mathcal{N}_{\text {new-block }}\right\}$ (number of generated information blocks). Specific values for communication efficiency depend on many variables at this point, but our objective is only a comparative study between the different endpoint's lifecycle; thus, no values are shown in axes in Fig. 36. As can be seen, dynamic wake-up scheduling endpoints present the best efficiency, as their lifecycle is always synchronized with the severs, reducing the information losses and the link management and information consumption costs. Both, fixed-period wake-up model and exponential evolution wake-up endpoints present a variable behavior as costs tend to be similar but information losses grow as more blocks are generated. Anyway, exponential evolution wake-up endpoints are slightly better as they can adapt increasing or reducing the sleep period. Always-on endpoints present a low efficiency 


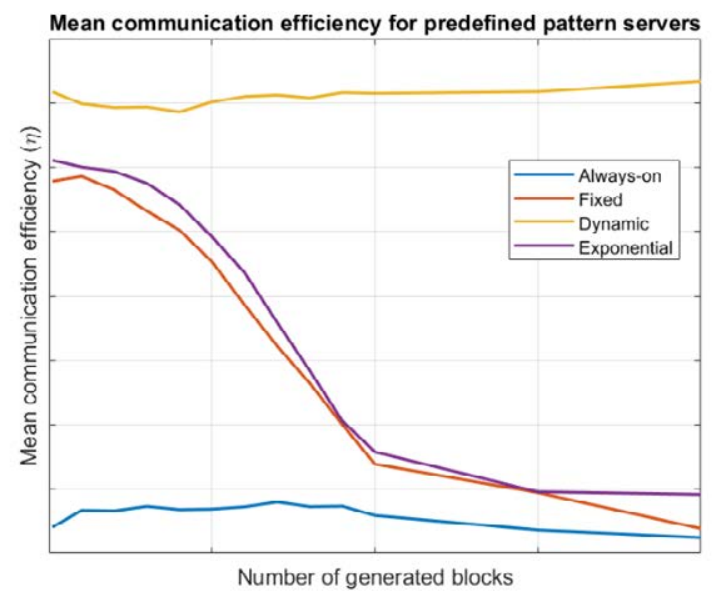

Fig. 36. Mean communication efficiency for predefined pattern servers

Fig. 37 shows the shows the evolution of the mean communication efficiency for the different endpoint's lifecycles, considering a sever following a Bernoulli pattern. Results are evaluated for different values of the block generation probability $p_{b e r}$. Always-on endpoints present a similar efficiency to other cases, but fixed-period wake-up model and exponential evolution wake-up endpoints have a variable behavior. For low block generation probabilities, the link management cost is much bigger than the value of the obtained information and efficiency is low. On the other hand, for large block generation probabilities the information losses go up and efficiency is also low. For values in the middle, a balance is reached, and efficiency is maximum. Besides, for low block generation probabilities, fixed-period wake-up endpoints are more efficient and for high block generation probabilities exponential evolution wake-up end points are better.

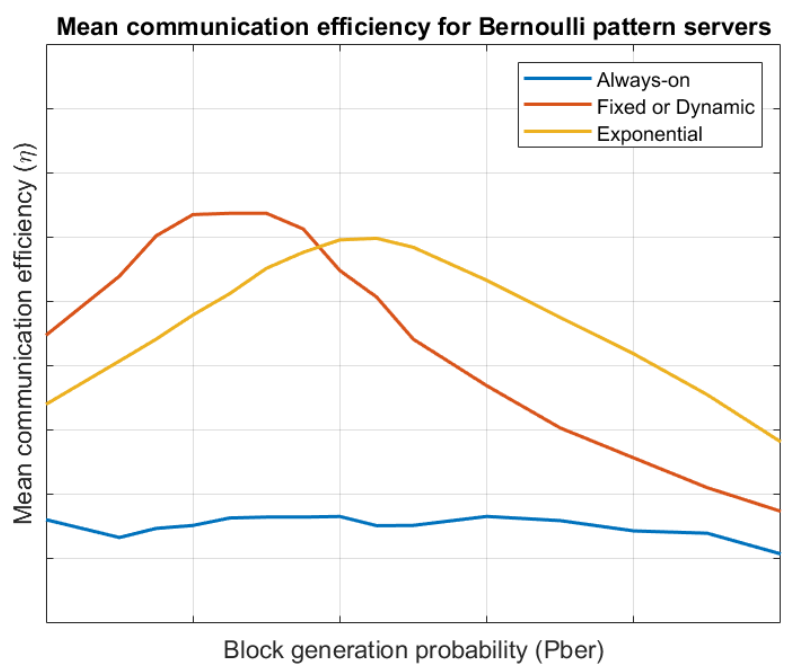

Fig. 37. Mean communication efficiency for Bernoulli pattern servers

Finally, Fig. 38 shows the evolution of the mean communication efficiency for the different endpoint's lifecycles, considering a sever following a Poisson pattern. Results are evaluated for different values of the mean number of generated blocks $\lambda_{\text {poisson }}$. It is 
similar to that obtained for Bernoulli servers, although in this case (globally), exponential evolution wake-up endpoints are more efficient.

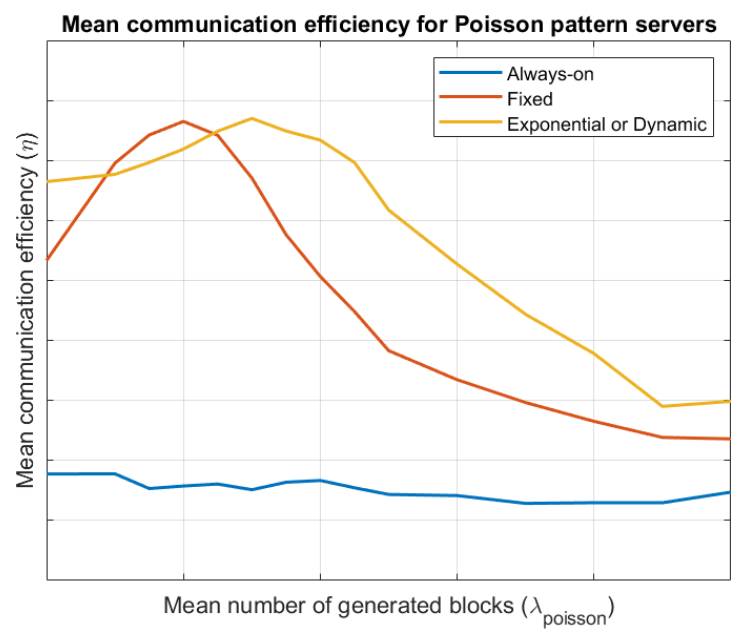

Fig. 38. Mean communication efficiency for Poisson pattern servers

On the other hand, we are evaluating the system efficiency depending on the compression algorithm. Fig. 39 shows the obtained results for different values of entropy. For low entropy information blocks, RLE algorithm is very efficient but as entropy grows the efficiency goes down. The same behavior is shown for Huffman algorithm, but it is a very costly algorithm (code is very complex and large, and large transitory data structures are needed) and only for a small number of situations its use is efficient. For high entropy information blocks the transmission in a raw format is finally more efficient.

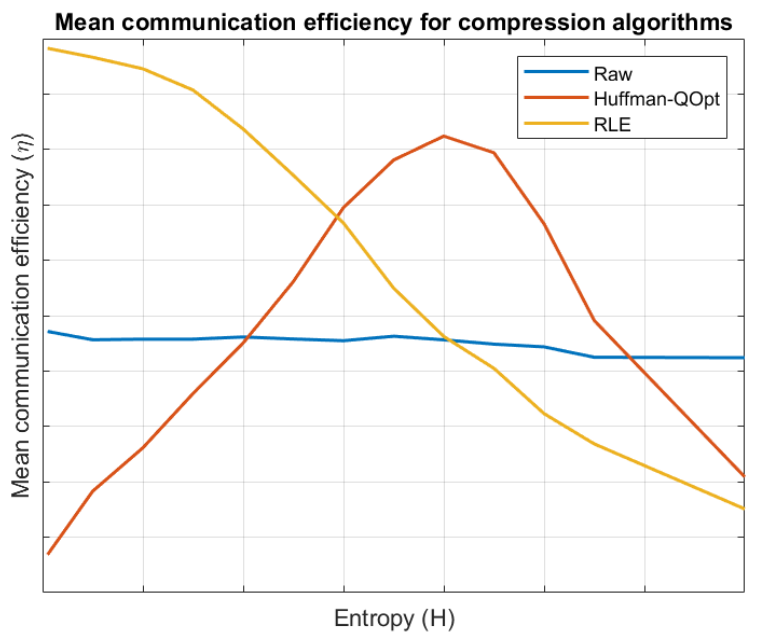

Fig. 39. Mean communication efficiency for different compression algorithms

Then, considering all showed results, the proposed algorithm is following some rules according the previous figures. Namely:

- By default, all endpoints are following an always-on lifecycle and using a raw transmission system. This approach, although it is the least efficient, allow us to 
collect information about the system behavior in the fastest manner, so a more efficient configuration may be easily selected.

- If low entropy information blocks are detected, the RLE compression algorithm will be selected. For high entropy blocks, raw transmission will be employed; and for intermedium situation the Huffman Q-Opt algorithm will be configured. Considering Figure 5, and the fact that entropy ranges in the interval $[0, k]$, Table 12 shows the proposed operation limits.

Table 12. Application limits for each compression algorithm

\begin{tabular}{|c|c|}
\hline Compression algorithm & Application limits \\
\hline RLE & $H\left(b_{i}\right) \in\left[0, \frac{k}{2}\right]$ \\
\hline Raw & $H\left(b_{i}\right) \in\left[3 \frac{k}{2}, k\right]$ \\
\hline Huffman Q-Opt & $H\left(b_{i}\right) \in\left[\frac{k}{2}, 3 \frac{k}{2}\right]$ \\
\hline
\end{tabular}

- For predefined pattern servers, a dynamic wake-up scheduling will be negotiated with the server. If it is not available, an exponential evolution wake-up model will be employed

- For Bernoulli pattern servers, two different situations are clearly shown (see Fig. 37). For low values of the block creation probability (for example, $p_{\text {ber }}<0,5$ ), the fixed-period wake-up model is more efficient. In any other case, the exponential evolution wake-up model will be employed by endpoints.

- Finally, for Poisson pattern servers, an exponential evolution wake-up model will be employed by endpoints.

Now, in order to detect the server configurations and, then, change dynamically the endpoints' configurations to increase as much as possible the communication efficiency, we are using the Bayes theorem (36). It is important to note that server may not be aware about certain behaviors inherit from managers (such as the information generation rate).

$$
p\left(\text { server }=P A T \mid Q_{i}\right)=\frac{p\left(Q_{i} \mid \text { server }=P A T\right)}{\sum_{\forall T} p\left(Q_{i} \mid \text { server }=P A T\right) \cdot p(\text { server }=P A T)} p(\text { server }=P A T)
$$

All endpoints may easily calculate the described costs, $Q_{i}$ (such as the information value $Q_{\text {info }}$ ) considering the proposed cost functions, and the real resource consumption and/or number of received information blocks. Then, after collecting data for a certain time period, it is possible (using previously described expressions) to evaluate the probability of the server to follow a certain pattern $P A T$, i.e. $p\left(Q_{i} \mid\right.$ server $\left.=P A T\right)$, known the costs $Q_{i}$. Thus, using the Bayes theorem and the proposed expression (36) it is evaluated the "posteriori" probability of a server to follow the pattern PAT. For this calculation we are considering all server's pattern equally probable, so $p($ server $=P A T)=$ $\frac{1}{3}$.

As three different costs related to the sever pattern have been defined ( $Q_{\text {info }}, Q_{\text {link }}$ and $\left.Q_{\text {consump }}\right)$, three different probabilities will be obtained. Each probability will be 
mapped into three different integer numbers, according two thresholds $p_{\min }$ and $p_{\max }$ (37).

$$
G\left(p_{i}\right)=\left\{\begin{array}{c}
1 \text { if } p_{i}<p_{\min } \\
2 \quad \text { if } p_{\min } \leq p_{i} \leq p_{\max } \\
3 \text { if } p_{i}>p_{\max }
\end{array}\right.
$$

Finally, an aggregated estimator will be employed (38) to rank all possible server patterns. The pattern with a higher mark is selected as the real pattern. Only patterns with a mark above the global threshold $G_{\text {threshold }}$ will be considered in the decision process.

$$
\begin{gathered}
G_{\text {total }}=G\left(p\left(\text { server }=P A T \mid Q_{\text {info }}\right)\right) \cdot G\left(p\left(\text { server }=P A T \mid Q_{\text {link }}\right)\right) \\
\cdot G\left(p\left(\text { server }=P A T \mid Q_{\text {consump }}\right)\right)
\end{gathered}
$$

In order to select the most efficient compression algorithm it is enough to evaluate the entropy of the received information blocks. If the central server allows stablishing a negotiation process, the compression method will be changed according to previously indicated rules. On the contrary, no change will be applied.

In order to avoid transitory effects (temporary behavior that are not stable nor permanent), the same result must be obtained in several different and independent evaluations, $N_{\text {eval }}$, across $N_{\text {hist }}$ sequential evaluations, to change the endpoint's configuration. Once efficiency is above a certain threshold $\eta_{\text {threshold }}$ the dynamic configuration process stops and will be run again if the efficiency monitoring procedure detects efficiency goes down.

Algorithm 2 codifies the described behavior.

\section{Algorithm 2. Dynamic communication efficiency optimization}

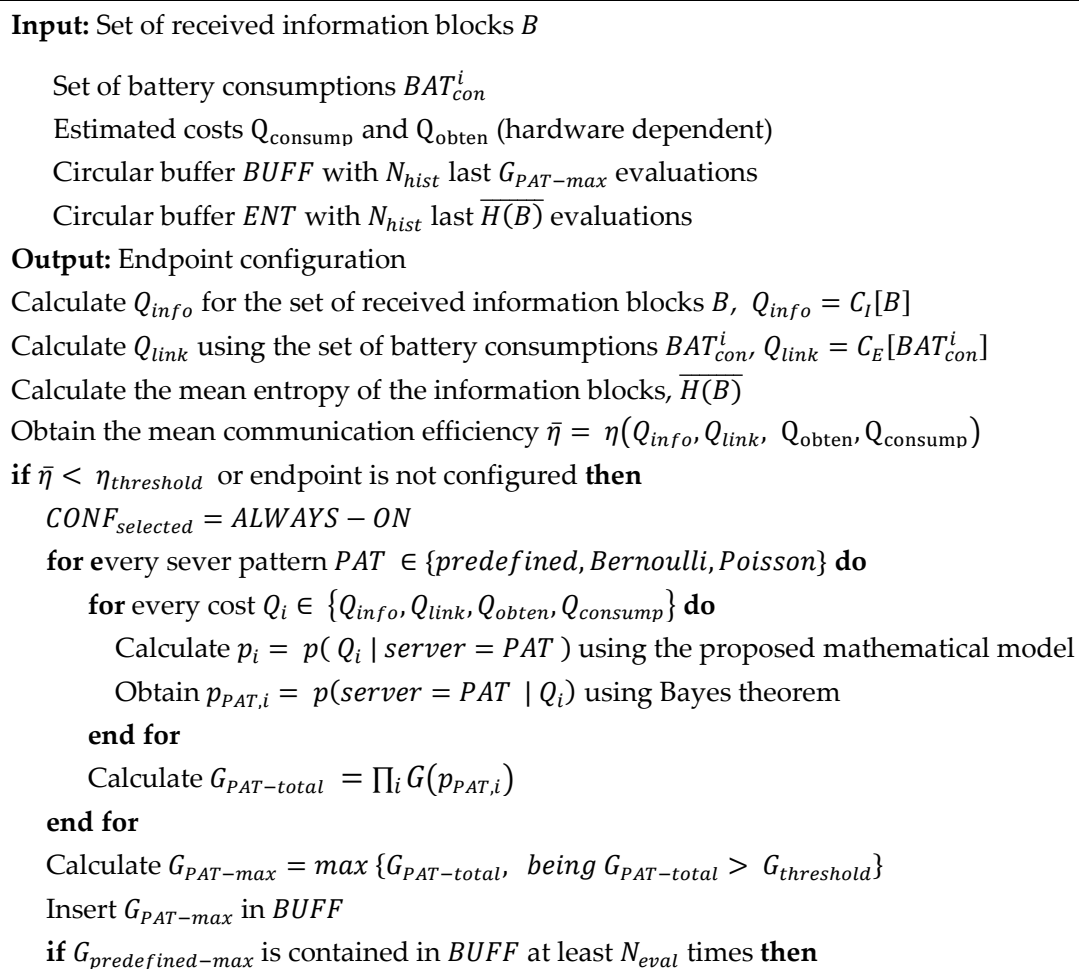




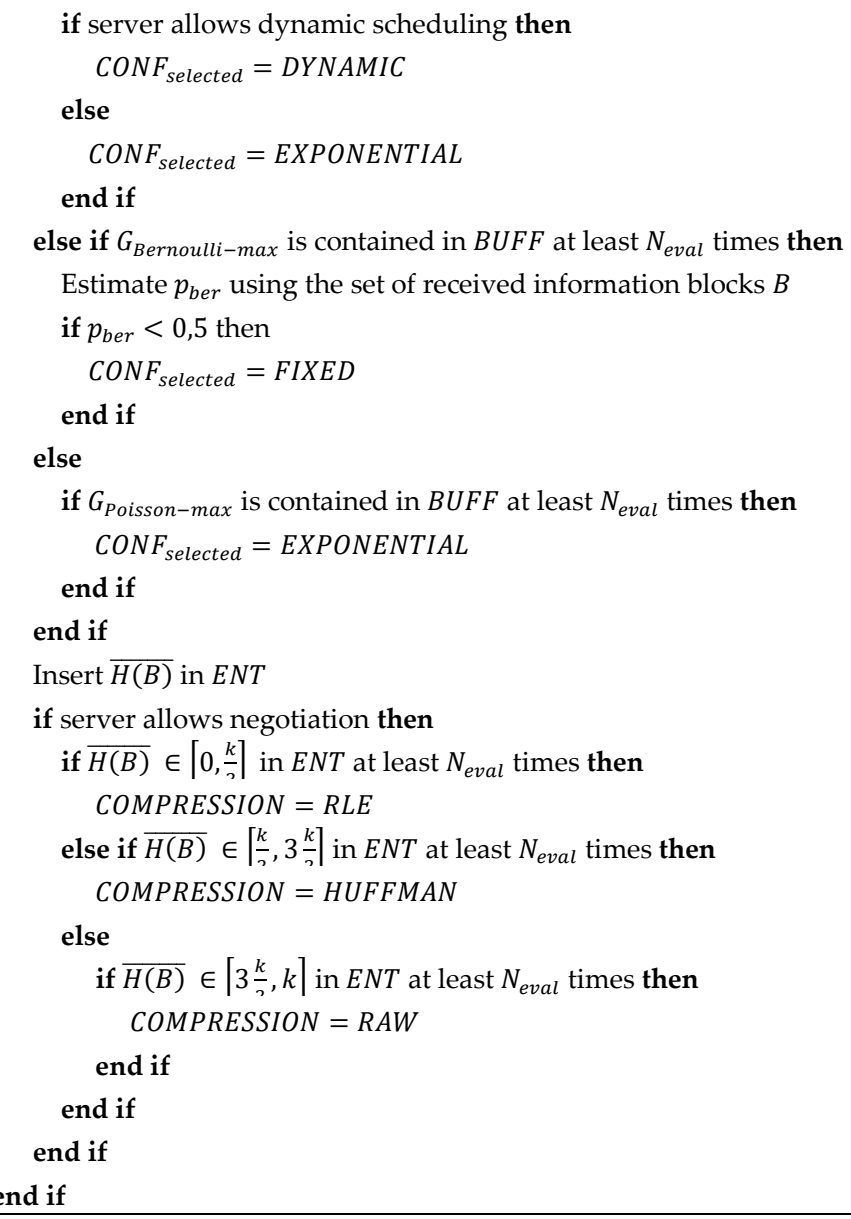

\subsection{Summary}

In this section, I propose two classes of algorithms to improve the battery utilization in a CPS. Devices are often battery-powered and placed in inaccessible locations that do not allow a continuous maintenance. Therefore, as the major impact on battery is done by the air time utilization of the wireless transmission, an optimized method of transferring and wake-up is developed.

Keeping a wireless device discoverable all the time can be avoided to increase the running time on battery with no power source connected. Thus, the addition of these transmission procedures is an important piece that every system will benefit of, as long as is composed by battery-powered elements.

This section proposes some methodologies to improve battery utilization in powered devices in order to extend their battery lifetime in a CPS and the new Internet of Things paradigm. Battery utilization techniques enable low computer resource elements to communicate wirelessly and battery powered with other components in longer periods and therefore reducing the maintenance of the global system.

Schedules in wake-up procedures imply benefits in both, shorter air time utilization and lower battery consumption. In this way, knowing the server model that best fits into 
the system is required to select the most appropriate implementation between the proposed models.

As well, the compression algorithms are a good choice in systems where the information transmitted to the final devices is big enough to produce a battery impact whether or not the information is compressed. This manner, a selection between more computational efforts or more air time utilization is the choice to do in order to reduce the battery draining to a minimum. 
8. OPTIMIZING COMMUNICATION EFFICIENCY IN CONSTRAINED ENDPOINT DEVICES 


\section{Validation in proposed application scenarios}

This section describes the application of the thesis contributions to different frameworks designed to validate the proposals of this work. Specifically, the service process architecture for unattended and interoperable devices have been applied in several testbed infrastructures that have been evolved during the time this PhD Thesis was written. This allowed the validation of the proposed methods in several environments, with a final evaluation in a real-world deployment as a smart shelf display system. The configuration of the system in this last scenario were made by nontechnical users and an evaluation has been made to provide also the perception of the technology improvements achieved. Other proposals, which were not validated in real scenarios, have also been theoretically validated in simulation environments. The methods described in this work have been validated through a series of measure and analysis of different indicators.

As a result, the behavior of the different implementations was validated by the implementation of different scenarios. The design and selection of these evaluation frameworks will be discussed in the following sections, as well as the analysis of the obtained results in each case.

\subsection{Validation frameworks}

I have defined several frameworks to validate the proposals of this PhD Thesis work. This section describes the aspects and the objectives of these frameworks in order to obtain a global visualization of the validation process used in this work. The presentation and the analysis are done from a chronological point of view, through research activities associated with the author's doctoral studies. The research activities have been developed in order to validate the contributions described in the previous sections are highlighted in bold. This chronological order does not necessarily correspond to the order of the presentation of the sections in this thesis. 
The first framework presented validates Section 4 proposal of a service generation chain of a CPS infrastructure. In this first framework, with the help of a domain expert, a description of the desired processes, a control entity is generated to manage the service execution phases. Measuring the amount of the successful execution in a simulated environment, I validate the improvements over other traditional service control processes. In this framework, the Section 7 proposal is also added to monitor the relevant information and to provide feedback to the platform administrator. Additionally, a multi-site environment is simulated, and the on-site locations are supplied with hardware managers.

The second framework is driven by the need of a CPS with automated capabilities. This requirement is defined by $R E Q \# \mathbf{1}$ that rely on the capacity of a system that recognizes and adapts itself with a set of available devices in an automated way. To provide a validation to the Section 6 proposal of this document, several scenarios have been designed in order to gather different measures of the system response. Specifically, a smart environment is used to prove the QoS concept applied to the resource allocation process and validate the convergence of the self-configuration method. At this stage of the work, I wanted to prove the usefulness of the methodology in its general form, thus the scenarios developed are not brought because any special requirement or any specific application. As they are focused only in the validation of the proposal, the inclusion of real-time processes and human intervention are required to expand the uses of the infrastructure to wider uses.

The third framework is designed to simulate the environment of a CPS with wireless devices in which several interfaces can be tested against the algorithms and models proposed in Section 8 in order to validate the proposed algorithm for a optimal communication efficiency in constrained devices. Several battery powered devices are put into the system and tested with several models of traffic generating a continuous flow of requests, allowing to measure the improvements over the standard communication methods. In this stage, a first prototype of a real-world CPS is provided. A display infrastructure is deployed as an optimized shelf system with continuous display representing information. These wireless devices are used to measure the improvements of the proposed algorithms for battery saving methods.

\subsection{A process control system testbed}

In this first framework, I validate the proposed service control system described in Section 4. As a first approach to a improve a CPS service chain, one of the first contributions to this field in my research work was to provide tools to a system administrator to interact with the system in a convenient way and to offer a concise tool to manage the configuration phase of a CPS. This work was expanded to not only support the configuration phase, but a continuous integration of the system inputs was implemented. 
The framework proposed in this section aims to validate the service control process as an effective system to control and improve the traditional methods present in the literature, often related to the emerging Industry 4.0 paradigm. The system implemented was conceived as a simulation environment of a real industrial deployment, and was developed in a laboratory of the Technical University of Madrid, where several machines in a virtualization environment emulated a production system. A total of four machines were deployed. Three of them implemented four virtual machines using an Ubuntu 16.04 image. Each virtual machine was executing an emulation program that represented different production process in each real machine. The virtual entities were interconnected by a virtual LAN and exposed to the exterior with a common interface. The emulation program was developed using Simulink, a module of MATLAB.

Three industrial processes were developed and simulated in the virtual entities, each of one deployed in a different real machine. All the virtual machines of the same real server were executing the same program, and simulating therefore the same process. The developed process considered three kind of manufactures: logic gates, capacitors and diodes.

The hardware controller of each group was executed at the same host, whereas the hardware manager was connected by an Ethernet adapter and hosted in a dedicated computer. In this last computer, the publication and subscription broker were deployed.

The remaining components defined in the Fig. 11, were implemented using different cloud services, where the majority of the components employed the Amazon Web Services framework in order to support the service flow proposed.

The interface showed in Fig. 40 is developed using a simple HTML page with several text boxes in order to interact with the backend server. The FSM can be programed using the DOT language previously described. Activity description also can be uploaded using the same page.

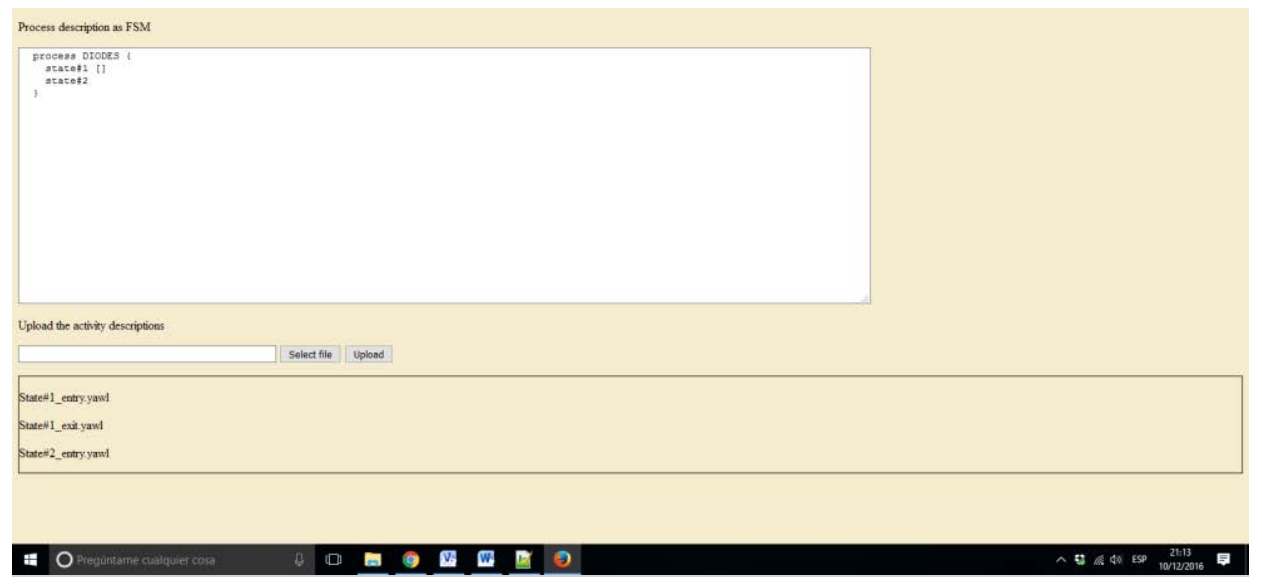

Fig. 40. FSM programing interface

A total of two different experiments were planned to provide a successful validation of the proposed service flow in the implemented architecture. 
In the first experiment of the process control system testbed, the programs used for emulating the service execution were designed to malfunction. Those failures must be addressed and solved by the proposed solution. Additionally, in order to measure the capability of the system to recover of such failures, different levels of severity were implemented. This first experiment was repeated fifteen times. A total of 180 processes were controlled by the proposed solution when the experiment finished, and all the gathered data were evaluated in order to prove the performance of the proposed method.

After the first experiment, a second infrastructure was implemented to contextualize and to value the results obtained. This second design comprises a supervisory system to control production entities. This logic was programmed in virtual SCADA platform with PLCs. In addition, OPC was selected as the communication protocol in order to simulate a real-world deployment of an actual CPS. The system was deployed in the AWS cloud infrastructure and programed with the openSCADA ('OpenSCADA: Main', n.d.) framework, as shown in Fig. 41.

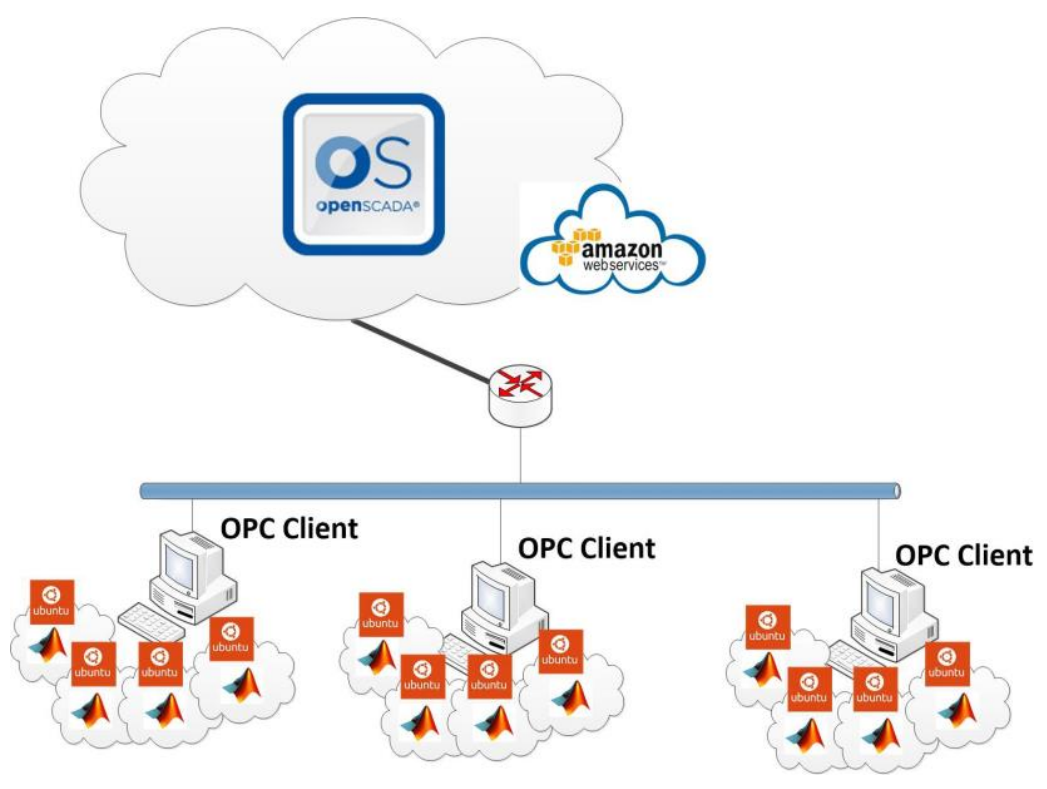

Fig. 41. Implemented supervisory system

The second experiment was planned to measure the response time of the service control flow proposed. To prove that the proposed solution is capable of provide a robust control of the service execution, the system should respond to the state change in a quick way in order to be able to recover the system successfully. The second experiment was divided in two parts.

In the first part of the second experiment, a constant number of service processes were executed. A number of ten processes were executed. Next, the events/minute rate was incremented in 10 events in each iteration. In order to achieve the increment, the virtual sensors adopted by the system were increased, as well as the virtual production systems. The rate was increased until the system became incapable of control the processes in a stable manner. 
The second part was performed by increment the processes in one unit, while keeping the events/minute rate fixed. Similar to the first part, the increment was stopped when the system failed to deliver a stable control.

\subsubsection{Results of the process control system testbed}

The combined results of the first experiment are shown in Fig. 42. The results show that in a $97 \%$ of the cases of the service executions, the proposed solution succeed in correct the malfunctions. In the remaining cases, the control policies applied could not control the processes and the system became blocked. These results are slightly better than other CPS control process methods found in the literature (Bordel Sánchez et al., 2015).

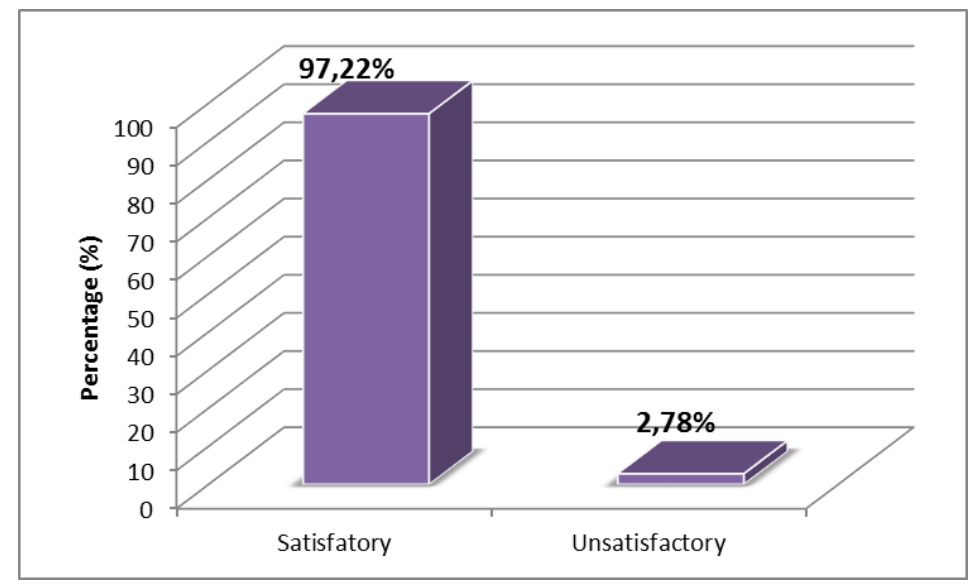

Fig. 42. Combined results

In this first experiment, the failures where assigned to a severity level and a various requirements depending of the classification of the process. Fig. 43 shows the failure causes that the control process was not able to recover.

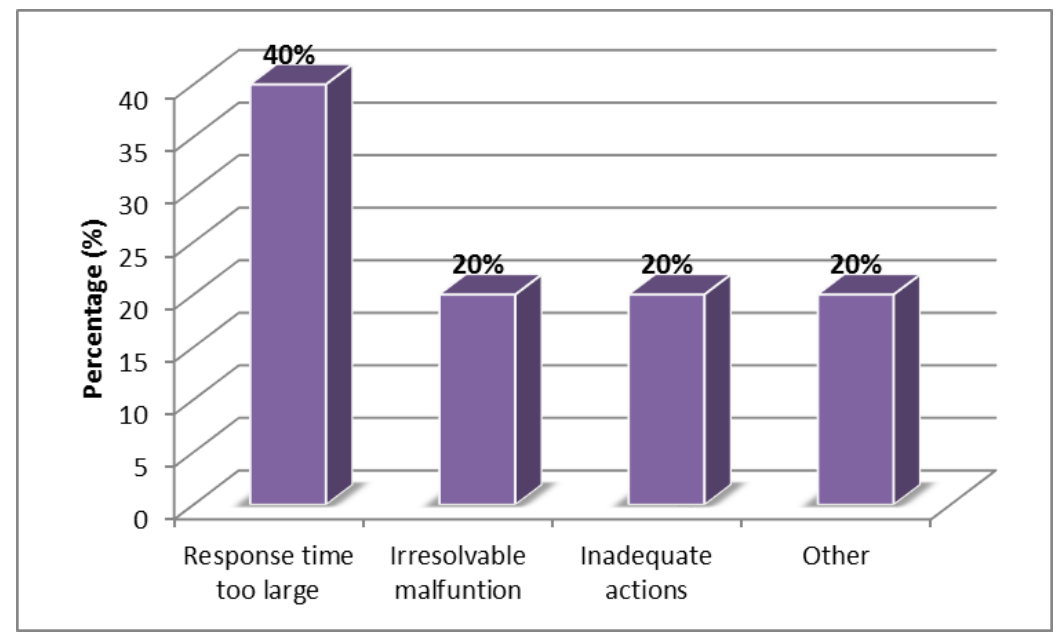

Fig. 43. Failure causes

With a $40 \%$ of the cases, the response time is responsible of the limitation of a correct failure recovery in the system. Although the malfunction was recoverable and the actions programmed in the control policies supported the resolution of the problem, a late response of the communication events were responsible of discard the 
resolution. Delays between the supervisory engine and the emulated systems cannot disseminate the events to apply the control policies in the required time, and therefore, the process response are not capable of correct the failures in the required time window. To reduce this time, a reduction of the control rules can be applied, so the hardware manager can react more rapidly in case of critical failures.

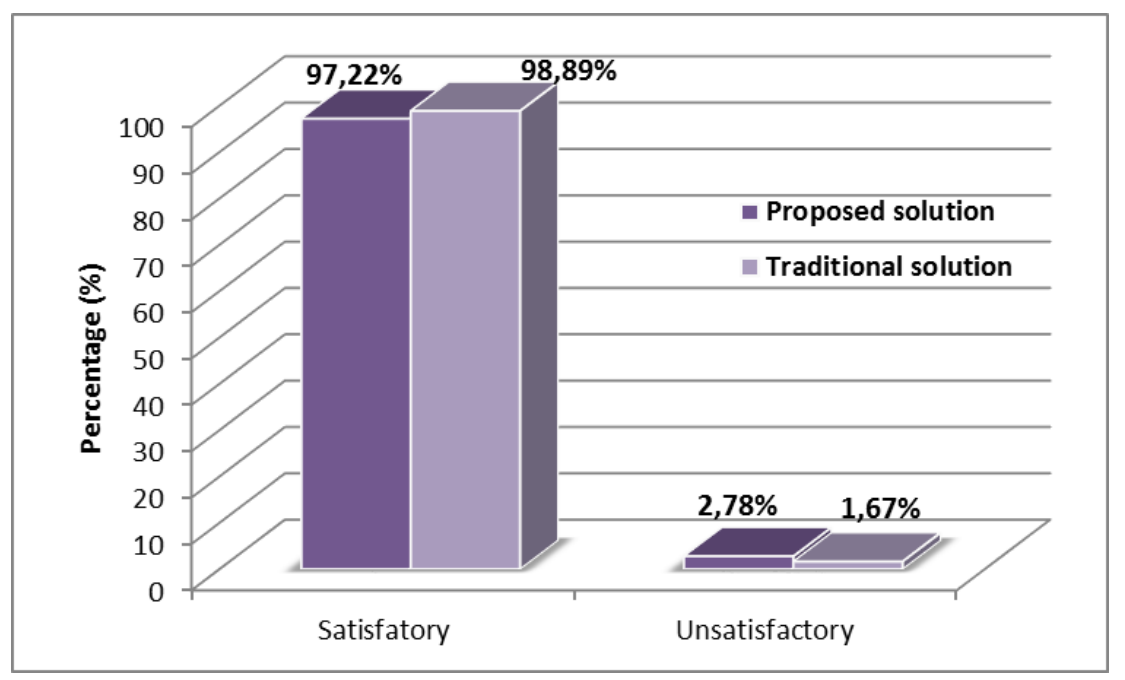

Fig. 44. Traditional system comparison

Apart from the resoluble malfunctions, in every system deployed in a real-world infrastructure, there are some situations that will not be able to correct in any case. Those situations must also be identified and extracted of our service control proposal. Some examples that can be found are external failures like supply or mechanical malfunctions. Other complementary control systems should be incorporated in order to mitigate those effects.

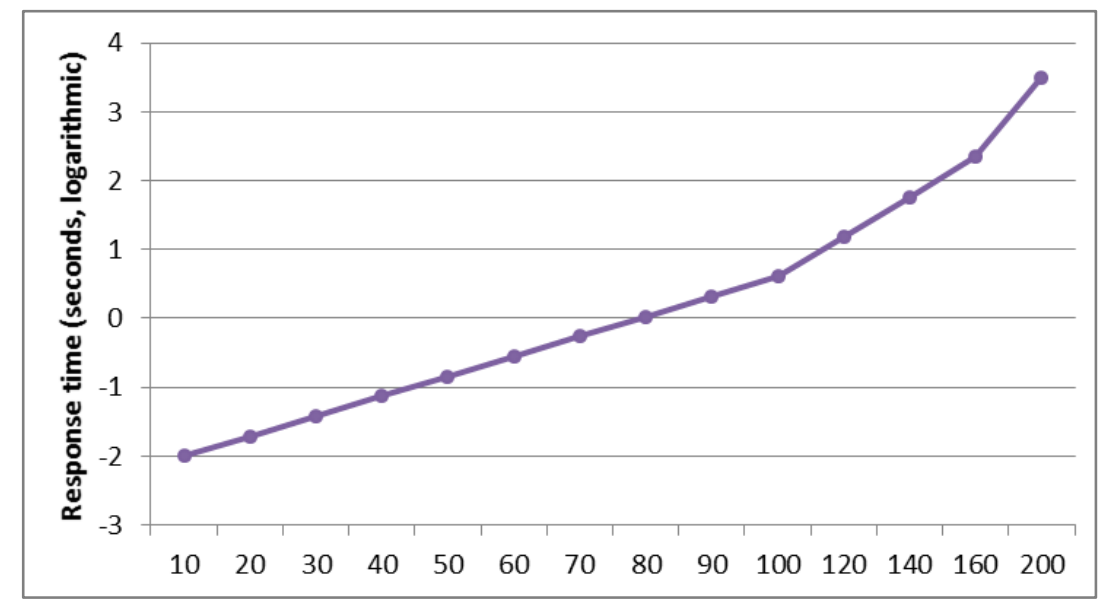

Fig. 45. Response time incrementing events/minute rate

As stated in the scenario description, these results were contextualized by compare them with a traditional control system, and the Fig. 44 shows the obtained outcome. The results show that, although the proposed method has a small improvement over other solution for service control, traditional SCADA system presents a better performance. In this analysis, the traditional service control process is capable of correcting $1 \%$ of the services better than the technology proposed in this work. 
Nevertheless, this improvement can be related to the variations produced by newer version of the SCADA developed over the last years and the experience gained in the real-world deployments of real devices.

Related to the second experiment, Fig. 45 shows the variation in the response time while incrementing the events/minute rate produced by the final entities (virtual sensors and production systems). The graph shows the mean of the temporal axis, as the time variable is a stochastic process, and presented in a logarithmic scale.

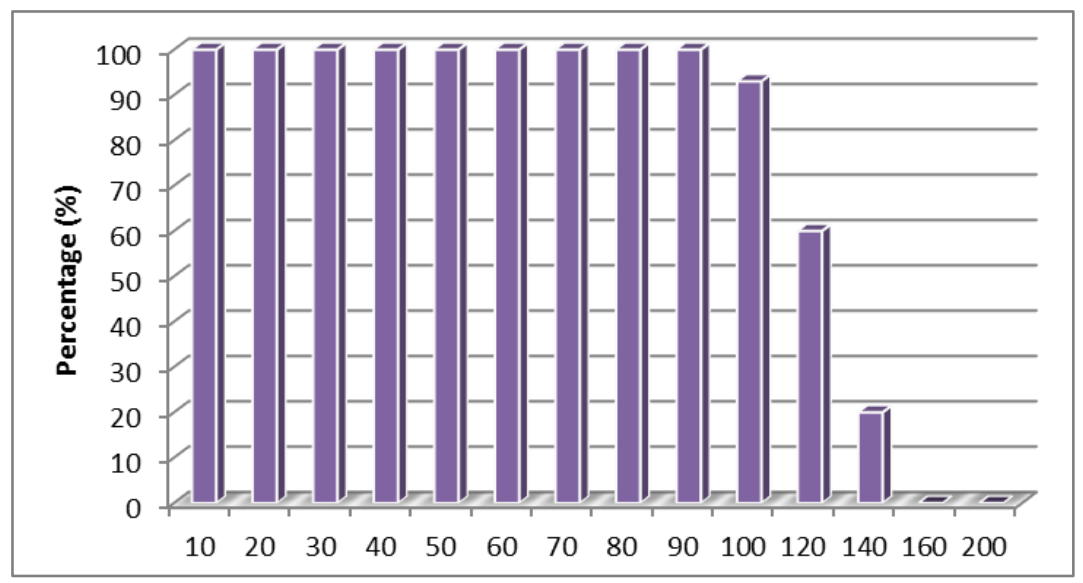

Fig. 46. Successful events percentage incrementing events/minute rate

In this case, the response time shows an exponential curve related to the events rate. This is shown as linear in the graph due to the logarithmic scale. A successful percentage of the events generated are processed in a satisfactory mode until the events reached the quantity of 100 events, where a drop can be observed. At this rate, the response time is about 100 seconds. Once the response delay exceeds one minute, the system starts to get blocked and the policies cannot be applied correctly. In this scenario, this situation occurs around a rate of 140 events per minute.

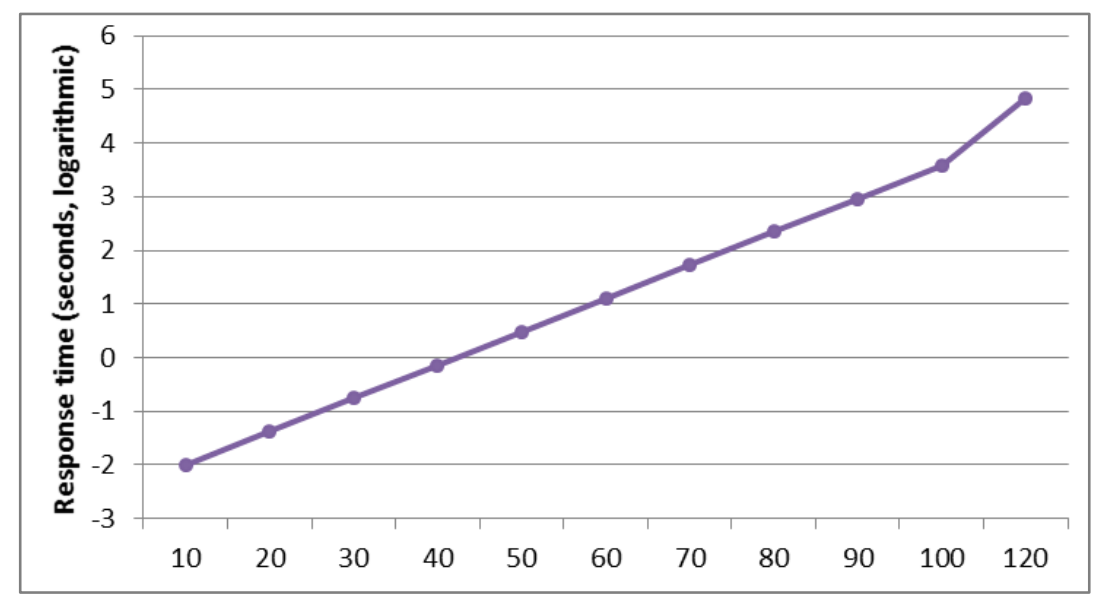

Fig. 47. Response time incrementing number of processes

In order to prove the real application of the proposed method, the values used in the experiment have been oversized to test the maximum rates of the system. As these values are very superior to real industry numbers, the proposal suits perfectly. Additionally, if a system requires a higher rate of events, more hardware managers can be added to 
obtain a more distributed architecture, gaining more computational power to address these situations.

Fig. 47 shows the second part of the second experiment. In this case, instead of increasing the rate of events per minute, the processes are increased, and the rate was leaved fixed. The graph shows a very similar tendency. Fig. 48 shows the percentage of the processes corrected in a successful way. This time, a drop appears around 50 processes. From there, a reduction can be observed until the processes reach 80 , where the system is blocked.

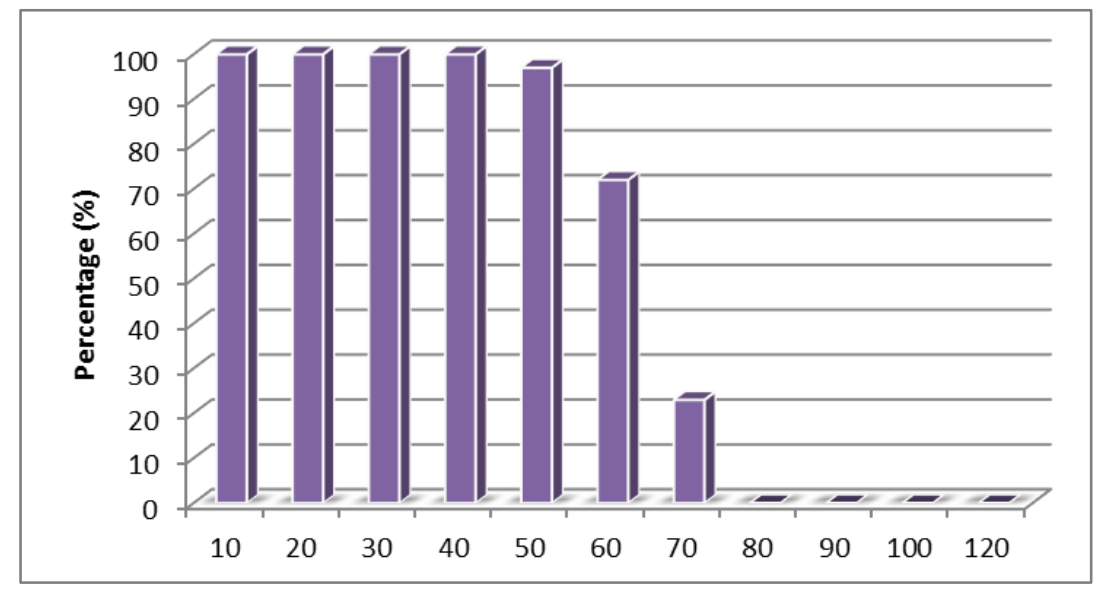

Fig. 48. Successful events percentage incrementing number of processes

As said in the previous case, more modules can be added to the system if the infrastructure needs to support more processes.

\subsection{Interoperable device configuration testbed}

In this second framework, several interoperable device configuration infrastructures are described to evaluate the validity of the proposed implementation described in Section 6.

To validate the solution and prove that there are improvements over other previous work in the same field, a set of scenarios are implemented and some considerations assumed:

- The adaptation of the system based on the QoS concept is implemented. The service composition procedures are required in order to obtain and update the list of the available services on the system, thus this composition of information is only performed here using simple mathematical operations.

- Information of the different final devices are gathered using logical operations and binary data format. This allows to perform the adaptation in a quick succession reducing the time of the transmission to a minimum.

- The semantic composition of the services, as stated before, is performed on the initial configuration phase. 
The experimental process is validated using four experiments that are complemented. Firstly, a real deploy is defined in order to prove the real work of the solution, and secondly, a set of simulation is done to prove the system scalability. Evaluation processes allow to measure the required convergence time that the configuration stage needs, and also depict the medium of the time that the system needs to converge to a stable state where starts to react to changes in the CPS environment.

Although, a smart environment can comprise several kinds of devices, to achieve this validation, a smart environment with three types of devices was used: a RFID enabled glove (Bordel Sánchez et al., 2015), a worktable provided with NFC readers and LEDs for user notifications, and an autonomous wireless device with e-paper display and lowenergy communication for information presentation (Rivera, Alcarria, Andres, Bordel, \& Robles, 2016).

In this first experiment, the smart environment was deployed and the scenarios designed, explained and evaluated in the following manner. Two scenarios were used.

- The first scenario was made up by fifteen devices, five worktables, five autonomous displays and five gloves

- Second scenario was made up by only seven hardware devices, including two worktables, two gloves and three autonomous devices.

Each scenario was deployed with a succession of two phases.

First the system is powered on and the time employed in the self-adaptation process measured. Then the system was turned off. This was repeated ten times. The second phase starts by initializing the system and then the data is gathered while the system is running for three hours. Information of the different adaptation processes and the time used to converge in each process were gathered. A said in the definition of the method, selecting a refreshing period is key in the configuration parameters. Different values has been tested in the second scenario. Table 13 shows the processes acquired in each scenario, and the values selected.

Table 13. Values acquired in each scenario - Second phase.

\begin{tabular}{lcccc}
\hline \multirow{2}{*}{ Scenario } & \multicolumn{4}{c}{ Refreshing period } \\
\cline { 2 - 5 } & $\mathbf{5}$ & $\mathbf{1 0}$ & $\mathbf{3 0}$ & $\mathbf{6 0}$ \\
\hline First & 142 & 106 & 97 & 89 \\
Second & 248 & 173 & 145 & 109 \\
\hline
\end{tabular}

In the second experiment, I evaluate the progression of the time that the system last in converge, when the self-configuration method is applied in a number of physical elements. This time, instead a real deployment, a simulated environment was deployed due to the difficultness of perform a real experiment with large amount of people and devices. 
This simulation was designed with the help of the NS3 simulator. This simulator allows to implement each entity as an agent with the correspondent particularities and technology. With the data that was obtained in the first experiment, a more precise behavior of the simulated entities was done. Information about the humans that participated in the experiment also was included. A series of scenarios were deployed and evaluated. To obtain incremental results, only one element was included in the first scenario, and then the devices were added to a total of 1000. Each simulation was undertaken for three real working hours, and all the data about the adaptation process gathered.

The third experiment focused on measuring the evolution of the resources needed to the adaptation process when several devices are being added to the system. This experiment can look similar to the second one, but with some considerations. As the resources were measured, several sinks of information have to be added to control the signalization overhead that can happen in the system.

The procedure in this third scenario was the same as the described for the second scenario, including the number of devices added to the system.

Lastly, in the fourth experiment, the results obtained in the previous experiments are compared to traditional solutions regarding self-configuration and self-adaptation processes in service-oriented CPS. A semantic service composition process (nsnam, n.d.) is selected as it resembles the traditional way of transmit the description files to other components.

\subsubsection{Results of the interoperable configuration testbeds}

This section summarizes the results of the experiments described in the previous section. Those results are presented and analyzed.

In Fig. 49 can be seen the results of the first phase performed in the first experiment. The measurements indicate the temporal values in the $Y$ axis that were normalized by the maximum value. The important information can be depicted by the relation between the two scenarios.

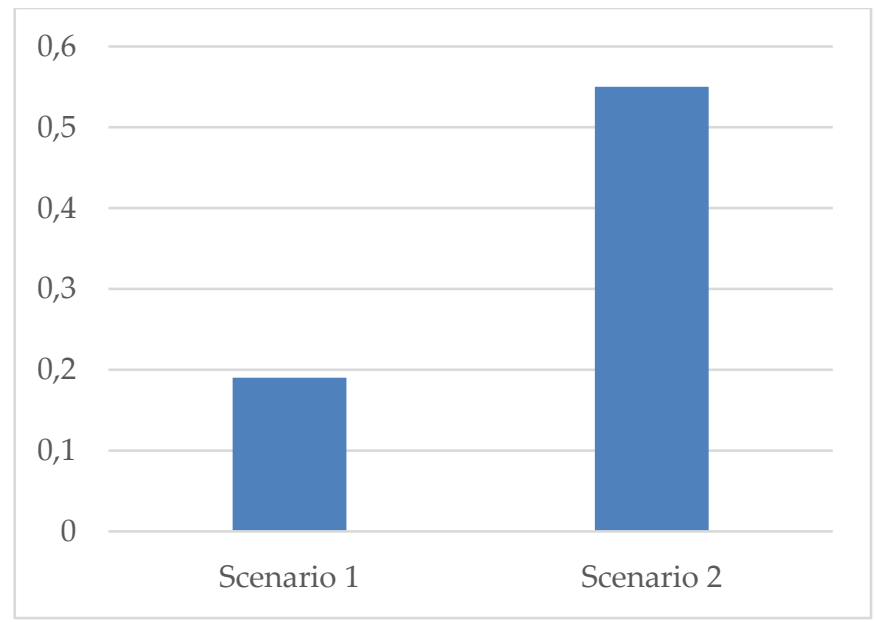

Fig. 49. Normalized initial convergence time (s) - first phase 
Fig. 49 shows the medium time resulted from the all repetitions performed. It can be seen that the maximum time is reached in the second scenario, when some humans were included in the experiment to prove the successful executions with social entities. The difference in the time is as much as $33 \%$ of the second experiment when only including hardware devices. This makes clear the increment in the needed time when humans are included and can be caused by several factors.

Human's states, per definition, requires more variables to configure than hardware devices, so the configuration phase must be superior in this scenario. Another identified factor refers to the hardware controller used that is user-focused and running in a central server. This is not the case when only smart devices are included, as the controller can be run inside their logic. This causes the communication delay to increment in the last scenario.

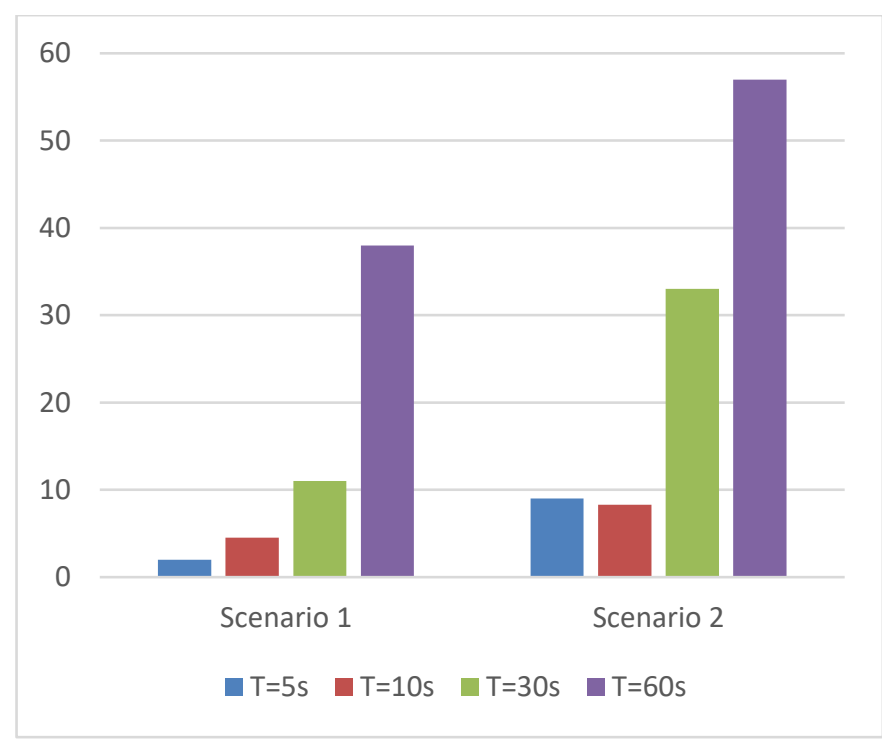

Fig. 50. Normalized initial convergence time (s) - second phase

Following, the second phase of the first experiment was measured. Table 13 summarizes the number of adaptation processes in each selected refreshing period. It can be seen that when the period is lower, the number of successful adaptations increment. These results prove that with a lower period, the fluctuations that can be found in a CPS with self-adaptation mechanism can be addressed by selecting the appropriate value.

In Fig. 50, the results measured on the second phase of the first experiment are shown. The $\mathrm{Y}$ axis represents the amount of time in which the system completes their adaptation. The two scenarios are shown, and their different refreshing time are also presented.

It is clear the relation that exists between the two scenarios, even with humans participating in the second one. Taking into consideration the results of the Fig. 49, some deductions can be extracted. The tendency observed can be proved as the second scenario obtains higher values in every selected value. This time, the convergence time around $40 \%$ lower from the first to the second scenario. 
If the tendency is eliminated, an important consideration can be extracted. The convergence time is not assured to be lower when a lower refreshing value is selected. This can be provoked by the measures that are being performed by the system that, in the second scenario cannot be completed in a short time because the human factors. Another consideration is that, when selecting a higher refreshing value, the convergence time is approximately the same. This remarks the independency of the value over the processing time but on the waiting intervals between the experimental measures.

Now, I analyze the results of the second experiment that has been performed. The measurements of the evolution of the convergence time when the number elements in the infrastructure grows, are indicated in the Fig. 51 . The results are normalized in order to obtain a relevant result from all the number of elements.

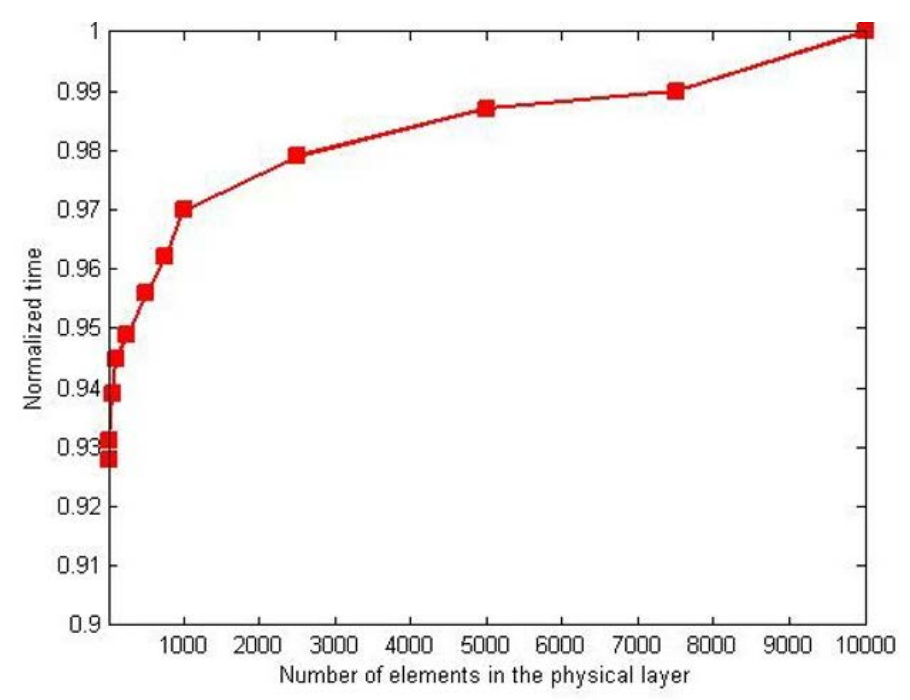

Fig. 51. Convergence time - second experiment

It is clear that the measured time are not influenced by the number of the hardware elements in the infrastructure. The values only present a $7 \%$ of variation between the lowest values to the highest, while the number of devices increases in several magnitudes high. This low variation can be due various facts. The architecture presents a topology in a tree form that allow the information flow to cover all the layers in an independent way of the quantity of elements present into the platform.

Additionally, the hardware manager acts as the gateway to the devices, so the information about the system are based on the performance achieved in its layer and then it rely all the business operation to a higher layer. Here, the codification of the status of each device being binary vectors helps the algorithms in order to ease the process. Therefore, the system proposed can be scaled, and the REQ\#6 fulfilled with the architecture described in this work.

In order to prove the scalability of the system implementing the self-adaptation processes, Fig. 52 shows the results of the third experiment. As with the convergence time, this experiment shows the normalized resource consumption versus the quantity 
of the hardware devices present in the system. The resource consumption is calculated based on the expression (39), and it measures the percentage of the transmitted information related to the configuration process in relation with the total.

$$
(\text { Configuration bytes } / \text { Transmitted bytes }) * 100
$$

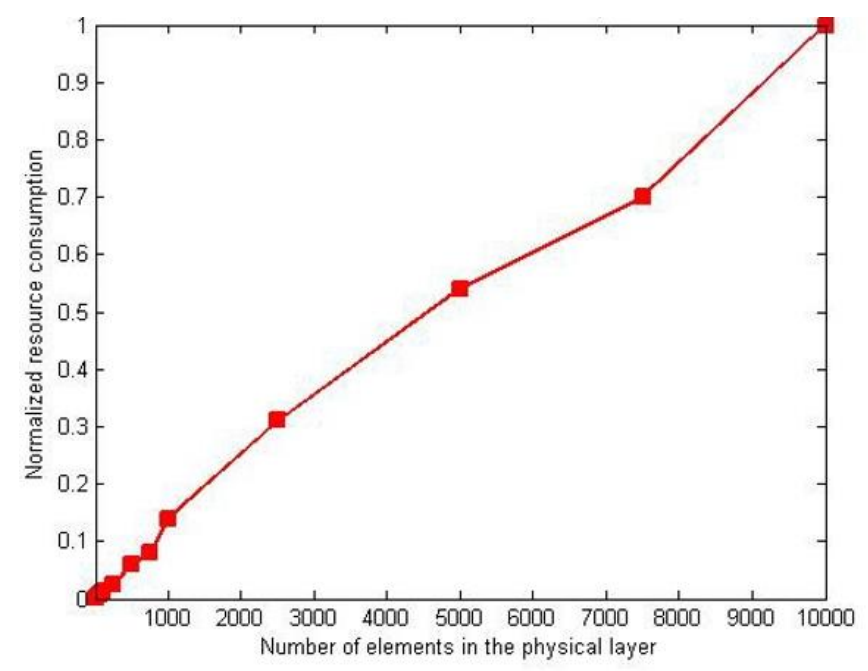

Fig. 52. Normalized resource consumption - third experiment

Fig. 52 shows the resource consumption increasing almost linearly with the increment of the hardware devices in the system. As each hardware device includes its own hardware controlled in this scenario -as imposed by the simulator used in this experiment- the result is logical. This results, although it may be imposed by the used technology, improves previous work where $n^{2}$ relations have been obtained (J. Wang, Zhu, \& Ma, 2013).

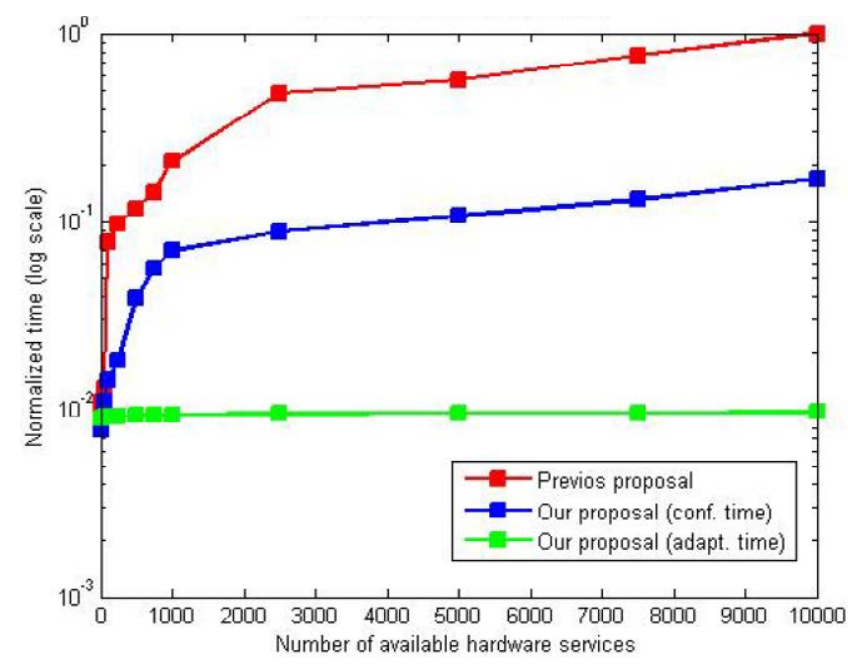

Fig. 53. Previous technology comparison

To finalize this first set of validation scenarios, a comparison is made between the data gathered in the convergence time and adaptation time experiments and a previous work that is using another method to provide similar results. Fig. 53 shows that the previous technology employs much more time to calculate the system adaptation 
process. The reduction in time gained by the inclusion of the proposed technology is clear and presents up to a magnitude order of reduction.

\subsection{Optimizing communication efficiency testbed}

Four experiments were carried out. The first group includes the three initial experiments and are based on simulation scenarios and tools. The second group, including only the fourth and final experiment, employs as main element an initial real implementation of the proposed solution.

Simulation scenarios and experiments are built using the NS3 network simulator. NS3 is a research simulation tool where scenarios and networks are described using C++ language. Results are obtained as a discrete sequence of events which may be processed and analyzed after finishing the simulation. This networks simulator considers three basic elements: information sources, communication networks and information endpoints. As seen, these elements perfectly fit the elements in our scenario (see Fig. 34). All simulations are carried out using a Linux architecture (Linux 16.04 LTS) with the following hardware characteristics: Dell R540 Rack 2U, 96 GB RAM, two processors Intel Xeon Silver 4114 2.2G, 2TB SATA 7,2K rpm.

Basically, the first three experiments are performed in the same scenario. A Smart Home, where connectivity is supported by WiFi solutions and where only one information source emulating the server behavior and functions is considered. The number of endpoints in the same scenario is variable, as well as the characteristics of content generated by the information source. All elements are considered to be configured at network and service level, to guarantee the connectivity and interoperability.

In order to implement the proposed configuration algorithm in the endpoints, and obtain relevant results, endpoints are connected to virtual instances running over the same operating system. These virtual machines are created and maintained through LXC technologies (Linux Containers) and the libvirt interface which enable the automatic creation and monitoring of these instances. Containers execute a unique process consisting of the described solution in Algorithm 1. Using ghost nodes and TAP bridges the output and inputs of these virtual instances is connected to the simulation elements representing the endpoints. In that way, it is possible to evaluate the performance of the proposed solution and enrich our simulation with real information. All the virtual machines are monitored in the use of their resources through the libvirt interface in order to feed the proposed algorithm with these data.

Using this scheme three different experiments were carried out. The first one considers fifteen endpoints in the scenario. Seven endpoints are receiving content with a low entropy (text) and eight endpoints are receiving data with a high entropy (images). The experiment studies the efficiency evolution in the proposed scenarios for the two different endpoints groups. Three cases are considered. In each case the sever behavior is changed: predefined fixed pattern, Bernoulli pattern and Poisson pattern. For each 
case, twelve simulations were developed and presented results are the mean of all obtained realizations. One hundred operation hours are simulated in each case.

The second experiment analyzes the delay required by our solution to react and change the endpoints' configuration to the most efficient scheme after a spontaneous change in the server or information characteristics. To perform this analysis, fifteen endpoints receiving all of them the same information are considered. Three cases are considered: change in the server pattern, change in the information entropy and change in the server pattern and information entropy at the same time. For each case, twelve simulations were developed and presented results are the mean of all obtained realizations. One hundred operation hours are simulated in each case.

The third experiment, the last one using simulation tools, is focused on comparing the proposed solution to existing proposals in the state of the art. The efficiency reached by the proposed solution is compared to the efficiency reached by a standard solution (Borja Bordel, Alcarria, et al., 2018). The same scenario than in second experiment was employed. Five hundred simulations were performed for each algorithm and maximum reached efficiency was measured.

The four experiment is quite different. In order to evaluate the performance of the proposed solution in a real deployment, it is developed a first initial system implementation. The proposed Smart Home consisted of a central server were a web server generated the information blocks. Then, five information endpoints were connected to this server thought a Smart Gateway implemented using the Samsung Artik 530 (Linux) architecture. Endpoints are electronic ink displays were images are shown. These endpoints are based on Artik 020 architecture and connected through Bluetooth wireless technology to the Smart Gateway. Fig. 54 shows the described deployment.

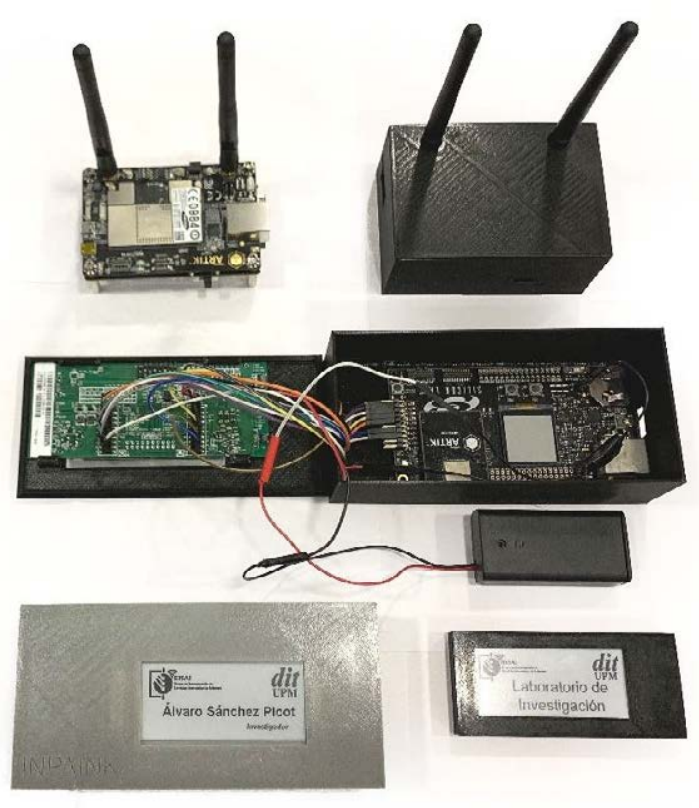

Fig. 54. Real implementation of information endpoints for CPS 
Artik 020 architecture is based on a high Performance 32-bit $40 \mathrm{MHz}$ ARM Cortex®M4 with DSP instruction and floating-point unit for efficient signal processing. It also includes a $256 \mathrm{kB}$ flash program memory and a $32 \mathrm{kB}$ RAM data memory. Using these resource-constrained devices it is evaluated the real consumption caused by the proposed solution. A very important aspect in our proposal is the possibility of the algorithm to be implemented and executed in resource constrained endpoints. The described deployment was operated for three days, and data about the resource consumption was collected through the debugging interface. In particular, the use of data memory (RAM), program memory (flash) and processing time is evaluated. The resource consumption was evaluated for different situations and configuration actions.

Table 14. Configuration parameters for the experimental validation

\begin{tabular}{c|c}
\hline Parameter & Value \\
\hline $\boldsymbol{N}_{\text {step }}$ & 10 \\
\hline $\boldsymbol{\lambda}_{\text {poission }}$ & 50 \\
\hline $\boldsymbol{N}_{\text {study }}$ & 5000 \\
\hline $\boldsymbol{p}_{\boldsymbol{b e r}}$ & 0,7 \\
\hline $\boldsymbol{T}_{\boldsymbol{s}}$ & 1 second \\
\hline $\boldsymbol{N}_{\boldsymbol{m i n}}$ & 2 \\
\hline $\boldsymbol{k}$ & 8 \\
\hline $\boldsymbol{h}$ & $7200 \mathrm{mAh}$ \\
\hline $\boldsymbol{B A T}$ & \\
\hline
\end{tabular}

Finally, for all four experiments, the Table 14 represents the value of all the configuration parameters described in the mathematical model.

\subsubsection{Results of applying the proposed algorithm}

Fig. 55 shows the results of the first experiment. As can be seen, the efficiency evolves according to a staircase function. This is caused by the double analysis described in our proposal: first the endpoint lifecycle and later the compression algorithm. In fact, calculations associated to compression algorithms (i.e. the information entropy calculation) are more stable in time, so the most efficient compression algorithm is selected much faster than the most efficient lifecycle, whose analysis has a more important stochastic character.

Thus, the first step in the stair corresponds to the compression method selection, and the second step to the lifecycle selection. Anyway, as can be seen, the proposed algorithm increases the efficiency operation above $60 \%$ in all cases. Even, for predefined severs (where the analysis considers few variables and then statistical noise is less relevant) efficiency reaches up to $90 \%$ (approximately). On the other hand, as Poisson servers are studied with expressions where probabilistic variables have a higher weight, statistical noise in this case is higher and present greater fluctuations. Besides, in this case, time required to obtain the most appropriate lifecycle is also higher than in any other case (and minimum for predefined servers where probabilistic variables have a smaller 
impact). On the other hand, in endpoints receiving messages with a high entropy, the time required to select the most efficient compression method is much smaller than in endpoint receiving low entropy messages. That is caused by the behavior of entropy function (logarithmic) which is more stable as the independent variable goes up. Finally, in some situations (see "Poisson server, high entropy" figure) temporary states may appear caused by false convergences (which are lately corrected).
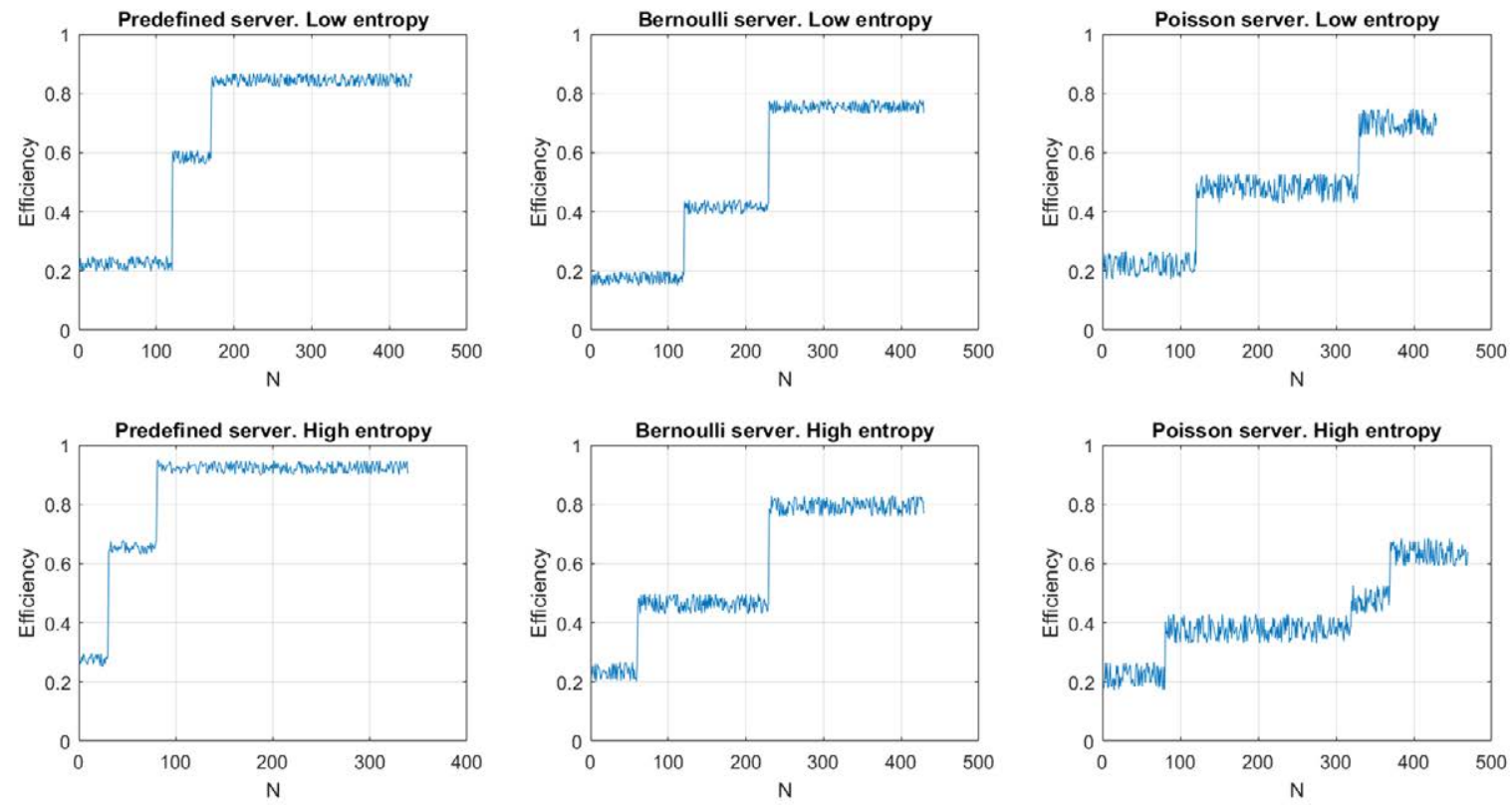

Fig. 55. First experiment: results

Fig. 56 shows of the results of the second experiment. As can be seen, changes in the information blocks' entropy are solved much faster than any other change; approximately $50 \%$ faster than changes in the server pattern.
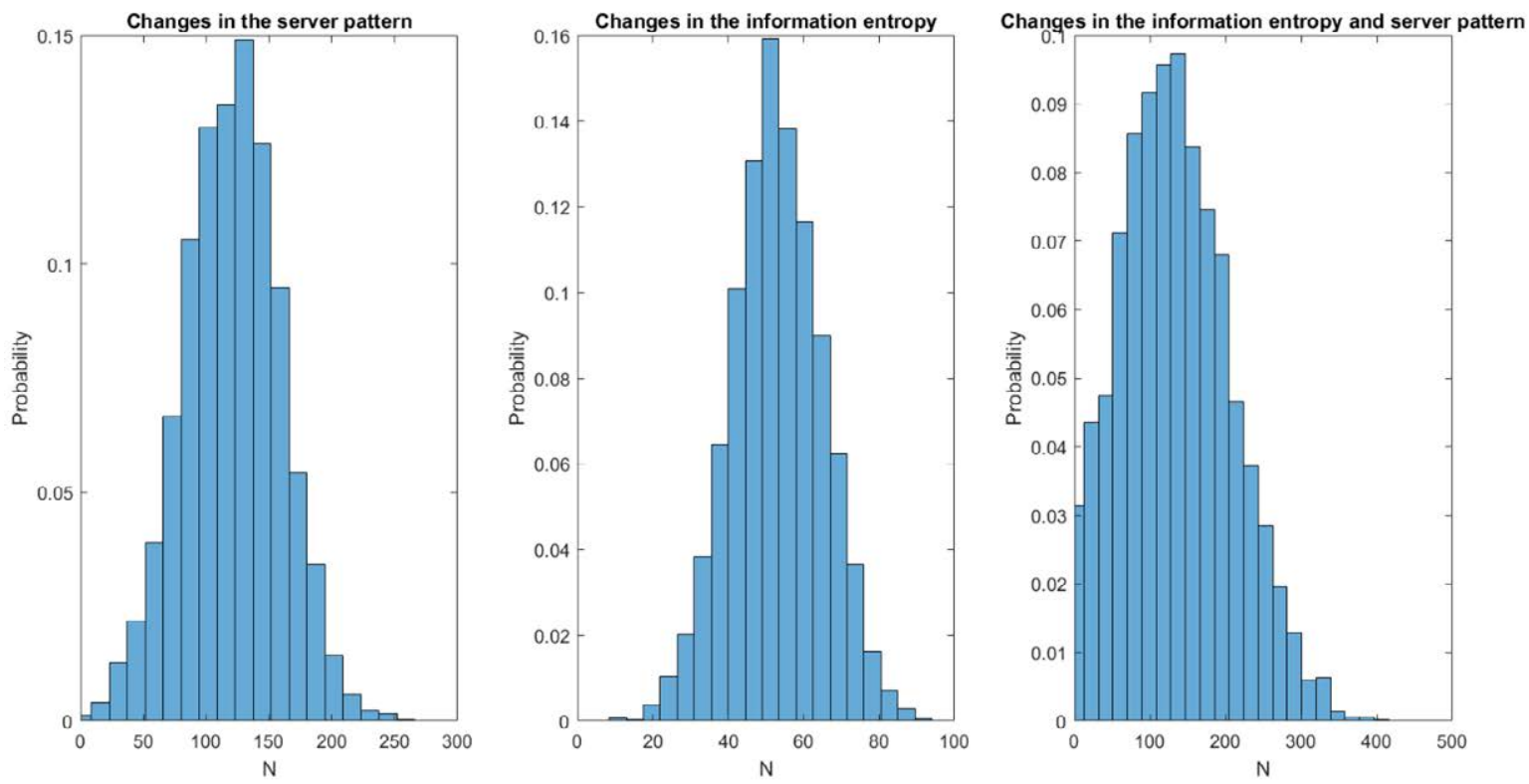

Fig. 56. Second experiment: results 
In standard time, changes in the information entropy are addressed in, approximately, 50 seconds, while changes in the sever pattern require around one hundred twenty (120) seconds. Dispersion (jitter) is also higher for changes in the server pattern, although it is especially relevant for situation when server pattern and information entropy suffer changes at the same time. In particular, situations where both changes occur together present a dispersion 100\% higher than any other situation.

Fig. 57 presents the results of the third experiment. Maximum reached efficiency is evaluated for different simulations, showing an increase up to $70 \%$ when employing the proposed solution. However, the main difference is the probability distribution for each case. Solutions in the state of the art are nor focused on a future efficient operation, so the maximum reached efficiency is a totally random value with a uniform distribution, and almost every possible value has a non-null probability. On the contrary, using the proposed solution, maximum efficiency is a Gaussian distribution with a quite low dispersion and centered around $\eta_{\max }=0,7$ (approximately). As can be seen, it is a very relevant improvement.
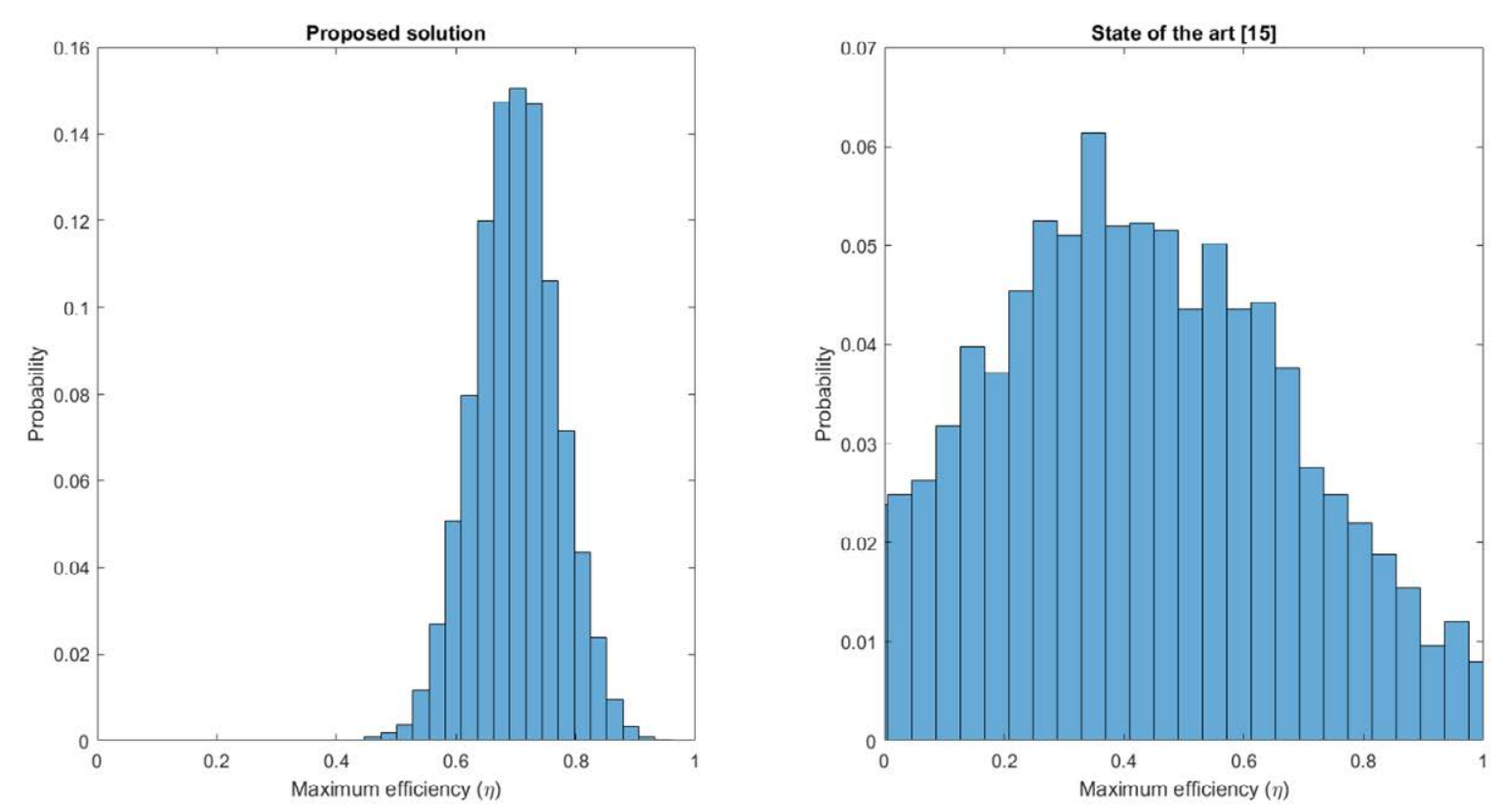

Fig. 57. Third experiment: results

Table 15 shows the results of the fourth and last experiment. As can be seen, the use of the space program, although is relevant, is acceptable or resource constrained endpoints. Moreover, the use of memory RAM is low (below 20\%), which fits the requirements of endpoints in CPS. Besides, the consumed processing time is always below three seconds per actualization (execution). 
Table 15. Fourth experiment: results

\begin{tabular}{|c|c|c|c|}
\hline $\begin{array}{c}\text { Configuration action } \\
\text { Predefined server to } \\
\text { Bernoulli server }\end{array}$ & $16 \%$ & $34 \%$ & $\begin{array}{c}\text { Processing time to perform } \\
\text { an actualization }\end{array}$ \\
\hline $\begin{array}{c}\text { Predefined server to } \\
\text { Poisson server }\end{array}$ & $18 \%$ & $34 \%$ & $1.9 \mathrm{~s}$ \\
\hline $\begin{array}{c}\text { Bernoulli server to } \\
\text { Poisson server }\end{array}$ & $18 \%$ & $34 \%$ & $1.9 \mathrm{~s}$ \\
\hline Entropy increasing & $12 \%$ & $34 \%$ & $1.5 \mathrm{~s}$ \\
\hline Entropy decreasing & $12 \%$ & $34 \%$ & $1.5 \mathrm{~s}$ \\
\hline
\end{tabular}

\subsection{Summary}

During this PhD Thesis work I have not limited to perform a theoretical work. Instead, the proposed contributions have been validated by defining three real scenarios whose problems are similar to the goals covered in this thesis work. The contributions made to meet these objectives have been implemented in the defined scenarios and the detected problems have proved resolved or reduced.

To conclude the first testbed scenario, the solution proposed has been analyzed and validated based on the NIST reference, see Fig. 8, which has been modified accordingly to the specifications and requirements described in this PhD Thesis. Although the process control proposed is focused on CPS infrastructure, it can be ported to the Industry 4.0 paradigm in order to provide process execution capabilities to the final devices. The process can be seen as a bottom-up process, as the hardware devices must be supervised by components sited in a higher layer, and allowing more granular control of the whole process.

In essence, the solution incorporates the domain expert figure to the configuration stage to provide a process model. This model is used to control a supervisory engine in charge of replicate a finite state machine applied to the execution control. The model created by the domain expert needs to be transformed in order to be understandable and executable, and then employed to apply the control policies into the system. All the process is monitored, and the transformation is described.

Second testbed validated the proposal described in Section 6, where an interoperable device deployment is done, and the system is started with the auto-provision method proposed. The testbed analysis was divided in four experiments, each of one with the objective of measure a different process. The separation of the different experiments has been proved as a successful way to complement the results of the global interoperable device methodology, and at the end, to prove the validity of the proposal. 
The different experiments showed a combination of real and simulated deployments that were composed by several devices. These devices were developed in previous work and provide a base for the test environment. It is worth to note that although the majority of the devices are autonomous, five smart gloves were used, incorporating to the experiments the human component that the nowadays CPS require.

The results of the second testbed proved that the proposed method provides similar results that other traditional proposals related to the configuration time, my proposal reduces the time that the system uses to calculate the adoption process.

Related to the third testbed, where a real deployment was made in order to test the entire system, the proposed algorithm presented in Section 8 was analyzed and validated in depth. Also, the analysis was performed by the inclusion of the described monitoring system stated in Section 7. The validation in a simulation environment shows that the proposed solution offers a comparable performance related to the control engines present in traditional literature, but this approach fits better in the CPS paradigm and Industry 4.0 requirements.

In this work we propose a new configuration algorithm for endpoints in CPS, so that they can operate in the most efficient way according to the dynamic characteristics of received information blocks and central sever behavior. The proposed algorithm makes priori predictions using a mathematical model, where all involved costs in the information reception and consumption are identified and quantified. Different server patterns and information block types (presenting different entropies) are considered in the model, to select the most appropriate endpoint lifecycle and compression method to increase efficiency as much as possible.

Priori predictions are corrected using the Bayes theorem with real measurement about the real resource consumption. The proposed solution is complementary to any other installed configuration solution to guarantee the system connectivity and interoperability.

In order to validate the proposed solution an experimental validation was carried out using simulation scenarios and real deployments. Results show a good performance of the proposed solution and a relevant efficiency increase in the system operation in comparison to previous proposals. 


\section{Conclusions and Future Works}

This $\mathrm{PhD}$ Thesis is motivated by the need to improve the current cyber-physical system scenarios where devices with minimum maintenance is required. This involves a rethink of the processes that nowadays have this CPS in order to provide novel solutions to the service process creation, device integration and device handling.

This section summarizes the contributions of this $\mathrm{PhD}$ Thesis work and justifies the validation of such contributions to meet the defined objectives. It is also discussed the novelty of applying this contribution compared to the state of the art.

Finally, a section of planned future works is presented, mainly related to the search, modeling and validation strategies to improve prosumer success.

\subsection{Conclusions}

This PhD Thesis is motivated by the current interest in the CPS paradigm, as in every other smart environment, of trying to reduce the configuration and integration phases and to obtain a working system with every task automated. The traditional conception of a CPS lacks a standardized architecture scenario where all modules are integrated gracefully in order to obtain the required maintenance, although several components were designed to provide those functionalities.

This need arises after analysis of the state of the art in various areas, detecting unexplored zones and trying to integrate several layer functionalities in a complete scenario where all concepts could be tested and validated.

Fields like service control, service provisioning and control paths were the starting point from which a service model that focuses on the interoperability level was introduced. This soon evolved to a scenario assessment and a reference architecture was proposed, trying to have covered several aspects that were identified as key, to provide the final environment complex. However, a lack of a unified method of device provision in the so-called on-site locations, provoked several of problems that I tried to solve in this work. 
To solve the problems that I identified in Section 3, where an analysis of the state of the art in the critical areas, I defined a service assessment model which enables the propagation of a service created in the management plane to the devices involved in the process. Considering that the creating of a new service is the responsibility of a user role of the platform, the needed propagation, and the capabilities requirements that the newly created task will employ, is specified. Moreover, with a service creation process, an important step in the initial phase of the system is the configuration phase, where I propose a workflow composed by several functional blocks. Those functionalities when working together can gather the basic information from the hardware level -hardware devices acting as sensors or actuators for the CPS- and start building in the integration chain a set of capabilities, and then, a set of services to be used by the application.

At this point, I use the concept of service-oriented CPS, as this enforces the focus of the proposed framework to the lifecycle of the services created and left behind the old linear structure of device and resource management lifecycle. The service-oriented CPS is based on top of the final devices that can change in the middle of a work phase: devices are often battery powered and wirelessly connected, so they are prone to suffer of battery run out and getting isolated of the network unexpectedly. This provokes the need of methods to reconfigure the system in these cases, and furthermore, the need for reconfigure again when those devices rejoin the same infrastructure.

The self-configuration for interoperable devices that this $\mathrm{PhD}$ Thesis work proposes to help the unitizing phase and the working phase of a system by understanding those changes and adapting the system. By the inclusion of the QoS information, the upper layers can evaluate the parameters compatibility that the new device is exposing to the system, and nevertheless, this information can be updated during all of the device coexistence in it. This allows the production level to be aware of certain changes in the behavior of the system. By updating the status of the services, applications can be aware of the current capabilities of the system and thus, optimize the use of resources accordingly.

After the definition of a smart environment, the analysis of the service-based framework shows that it is clear that the traditional problems on the SE based environments are depicted by the feedbacks loops that in most of the cases are predefined and pre-configured. With the identification of several problems that can be take place in the CPS environment, I have proposed a set of contributions to allow the mitigation of unwanted effects by the use of the current architectures. Concepts like battery powered devices and wireless transmission bring problems that I have tried to mitigate with the addition of several methods and procedures. With this, added as a dynamic process, the architecture presented achieve more complex and more powerful integration, even with the auto-adaptation mechanisms proposed in this work. This proposal fits in the group of mechanism that relies the low-level service definitions of the hardware level to the superior levels where it can be managed. With this background, I extracted a series of requirements that helped the design decisions in the 
next phase of the work: the definition of a reference architecture that englobes all the proposed integrations.

One important step of my work was the definition of a reference architecture for multi-site CPS, where the design of a complete system was made. Using a component per function approach, the resulting system is able to identify all the actors and modules integrated into the system and to perform tasks that are created and started from a central management entity.

After the definition of a smart environment, an analysis of the service-based frameworks it is clear that the traditional problems on the SE based environments are depicted by the feedback loops that in most of the cases are predefined and preconfigured. With the addition of a dynamic process, the abstraction achieved is more complex and more powerful, integrating in the loop the service creation and autodiscovery capabilities proposed in this work. The proposal of Section 6, fits in the group of mechanisms that rely the low-level service definitions of the hardware level to the superior levels where it can be managed.

With a focus on final devices, an algorithm to decide the best option to maximize the communication efficiency has been proposed. With the help of a mathematical model, a priori predictions are calculated to select the most appropriate transmission method and wake-up algorithm. Several server models have been considered to discern between different patterns. A theoretical analysis has been done to prove the benefit of the proposed algorithm. In this manner, the use of the Bayes theorem helps the calculation of the a priori predictions when working with predefined server models.

A real deployment focused on a smart shelf system was done, and the proposals tested to prove and to validate them. The proposed architecture was implemented and several locations simulated to interact with the management module. The system was composed by several sensors and smart displays acting as actuators in order to simulate a real world environment.

A stated at the beginning of this work, manage a flexible CPS with ongoing processes in a multi-site environment, can be challenging and requires scalable solutions that allow the addressing of the all-possible cases that can occur. CPShaveevolved from their origin as advanced embedded devices to a paradigm for the next generation of technological systems. In particular, systems to execute user-defined processes based on serviceoriented CPS have emerged as a promising field. Many proposals related to this topic may be found, although none of them truly allows users to define processes in a high level of abstraction, which later, may be executed in a traditional ad hoc network or pervasive computing infrastructure.

Therefore I have not limited myself to perform theoretical work. Instead, the proposed contributions have been validated by defining three real scenarios whose problems are similar to the goals covered in this thesis work. 


\subsection{Future Works}

This section exposes the planned works that are planned in the future, in relation to the contributions that are described in this PhD Thesis work.

As the CPS paradigm is growing every day, there are already candidates where the future hopes to base its development. In order to prioritize the works that have to be done in this field to get a more powerful architecture of a scalable, adaptable, heterogeneous and extensible CPS infrastructure, I have identified some relevant steps that I think are worth to take.

This work will include researching the current trends in terms of the architectural motivation to support the new technologies that arose recently, and that it is expected to disrupt the actual form of facing a device-based architecture. The addition to the mix of the devices that are capable of last years without human intervention, or the inclusion of connected devices in each miniaturized element, only complicate the design of a truly scalable infrastructure that supports the growing pace of the last trend.

Future works should address further challenges. Besides, as the proposed experimental validation only considers a first basic implementation of CPS (in a scenario similar to a multi-site environment in a small scale), future works should also evaluate the performance of the proposal in applications such as traffic flow management (large scale), electric power generation management and personalized healthcare devices. Finally, advanced studies about the performance of the proposed framework should also be carried out: Scalability of fault resiliency is parameters to be investigated.

Future works should also consider the proposal of particularized lifecycles for on-site management process and the definition of specific operations for service regeneration, update, etc. based on labels and selectors. A prosumer interface for the creation and configuration phase of the servicer workflow in an automatic way should be also interesting to be investigated in the future.

With relevant challenges that nowadays emerge in the future paradigm, concepts like $5 \mathrm{G}$ demand architectures that adapt themselves in a generic framework with great scalability that provides and impulse new methodologies, procedures and technology to provide people the information they require in a seamless experience.

In this way, the CPS paradigm can bring the improvements achieved in the last years in order to ease the change and the evolution of a global 5G framework. It is necessary, however, the need of research and finding a solution to various problems that I think a global infrastructure would require.

For example, the simple task of synchronizing service execution in a wider environment, can lead to synchronization problems along the entire system, and it can result in real-time disruptions in the service provision.

Transversal requites can be also a matter that worth to work. In this case, the requirements of the system can be consistent with the environment, but with the new trend of providing a holistic point of view (by means of dealing with the all the possible 
aspects), the field of application can be extended and new challenges found. To name some, I would focus on the incorporation of people to the process and encourage them to participate in the execution lifecycle, not only to provide actions to the system, but to take part into the tasks involved. 


\section{Publications}

During the course of this PhD Thesis, the author has made a number of publications, some of them directly related to the contributions and some indirectly related, but that, somehow, contributed to validate the ideas and proposed solutions.

\section{Publications directly related to the objectives of this thesis}

Publications that are directly related to the objectives of this thesis can be classified into the following areas:

In the context of the CPS service provision, where the author has proposed and validated the methodologies described in this work:

- Bordel, B., Alcarria, R., Sánchez de Rivera, D., \& Robles, T. (2018). Process execution in Cyber-Physical Systems using cloud and Cyber-Physical Internet services. The Journal of Supercomputing, 74(8), 4127-4169. JCR (2017): 1.532 Q2.

- Sánchez, B. B., Alcarria, R., Sánchez de Rivera, D., \& Sánchez-Picot, A. (2016). Enhancing Process Control in Industry 4.0 Scenarios using Cyber-Physical Systems. JoWUA, 7(4), 41-64.

- Sánchez de Rivera, D., Alcarria, R., Martín, D., Sánchez-Picot, Á., Bordel, B., \& Robles, T. (2016, March). Distributed Query Results and IoT Data in a PublishSubscribe Network Implementing User Notifications. In 2016 30th International Conference on Advanced Information Networking and Applications Workshops (WAINA) (pp. 778-783). IEEE.

- Sánchez de Rivera, D., Sánchez-Picot, Á., Alcarria, R., de Andrés, D. M., \& Robles, T. (2014, December). Smart dynamic pricing based on ECA rules and electronic ink labeling for retail. In International Conference on Ubiquitous Computing and Ambient Intelligence (pp. 468-475). Springer, Cham.

- Sánchez de Rivera, D., López, C., Alcarria, R., Martín, D., \& Robles, T. (2014, December). Smart Product Management in Retail Environment Based on Dynamic Pricing and Location Services. In International Conference on Ubiquitous Computing and Ambient Intelligence (pp. 171-178). Springer, Cham. 
- $\quad$ Bordel, B., Sánchez de Rivera, D., \& Alcarria, R. (2016, July). Plug-and-play transducers in cyber-physical systems for device-driven applications. In 2016 10th International Conference on Innovative Mobile and Internet Services in Ubiquitous Computing (IMIS) (pp. 316-321). IEEE.

- Bordel, B., Sánchez de Rivera, D., Sánchez-Picot, Á., \& Robles, T. (2016). Physical processes control in industry 4.0-based systems: A focus on cyber-physical systems. In Ubiquitous Computing and Ambient Intelligence (pp. 257-262). Springer, Cham.

In the context of providing a methodology for automated configuration processes in CPS environments, with special interest in ease the device incorporation mechanism and bootstrapping procedures:

- Bordel, B., Alcarria, R., Martín, D., Robles, T., \& Sánchez de Rivera, D. (2017). Selfconfiguration in humanized cyber-physical systems. Journal of Ambient Intelligence and Humanized Computing, 8(4), 485-496. JCR (2017): 1.423 Q3.

- Bordel, B., Alcarria, R., Sánchez de Rivera, D., Martín, D., \& Robles, T. (2018). Fast self-configuration in service-oriented Smart Environments for real-time applications. Journal of Ambient Intelligence and Smart Environments, 10(2), 143-167. JCR (2017): 0.878 Q4.

- Sánchez-de-Rivera, D., Bordel, B., Sánchez-Picot, Á., Martín, D., Alcarria, R., \& Robles, T. (2019, February). Sharing Device Resources in Heterogeneous CPS Using Unique Identifiers with Multi-site Systems Environments. In International Conference on Information Technology \& Systems (pp. 153-164). Springer, Cham.

- Bordel, B., Iturrioz, T., Alcarria, R., \& Sánchez-de-Rivera, D. (2018, July). CyberPhysical Sensors and Devices for the Provision of Next-Generation Personalized Services. In International Conference on Innovative Mobile and Internet Services in Ubiquitous Computing (pp. 479-490). Springer, Cham.

In the context of optimizing the communication efficiency and enabling constrained devices to optimize their operation in an automated manner:

- Sánchez-de-Rivera, D., Bordel, B., Alcarria, R., \& Robles, T. (2019). Enabling Efficient Communications with Resource Constrained Information Endpoints in Smart Homes. Sensors, 19(8), 1779. JCR (2017) 2.475 Q2.

- Sánchez-de-Rivera, D., Alcarria, R., Martín, D., \& Huecas, G. (2018). Proposal and Assessment of Algorithms for Power Consumption Reduction in Wireless Networks with E-Ink Displays. Journal of Internet Technology, 19(7), 2035-2046. JCR (2017): 1.301 Q3.

- Sánchez, B. B., Alcarria, R., Sánchez De Rivera, D., \& Sánchez-Picot, A. (2016). Predictive algorithms for mobility and device lifecycle management in Cyber-Physical Systems. EURASIP Journal on Wireless Communications and Networking, 2016(1), 228. JCR (2016): 1.529 Q3. 
- Sánchez de Rivera, D., Alcarria, R., de Andres, D. M., Bordel, B., \& Robles, T. (2016, January). An autonomous information device with e-paper display for personal environments. In 2016 IEEE International Conference on Consumer Electronics (ICCE) (pp. 139-140). IEEE.

- Sánchez de Rivera, D., Robles, T., López, J.A., Sierra de Miguel, A., Navarro, M., M. de la Cruz, J.A., Skarmeta, A.F., Iglesias Gómez, M.S. (2017). Adaptation of Ontology Sets for Water Related Scenarios Management with IoT Systems for a More Productive and Sustainable Agriculture Systems. SEMANTICS Workshops.

- Sánchez, B. B., Sánchez-Picot, A., \& Sanchez De Rivera, D. (2015, July). Using 5G technologies in the Internet of things handovers, problems and challenges. In 2015 th International Conference on Innovative Mobile and Internet Services in Ubiquitous Computing (pp. 364-369). IEEE.

- Bordel, B., Alcarria, R., Pérez-Jiménez, M., Robles, T., Martín, D., \& Sánchez de Rivera, D. (2015, December). Building smart adaptable Cyber-Physical Systems: definitions, classification and elements. In International Conference on Ubiquitous Computing and Ambient Intelligence (pp. 144-149). Springer, Cham.

- Bordel, B., Alcarria, R., Sánchez-de-Rivera, D., \& Jara, A. (2017, July). Low-Level Service Management in Cyber-Physical Systems. In International Conference on Innovative Mobile and Internet Services in Ubiquitous Computing (pp. 860872). Springer, Cham.

In the context of providing a social aware service provision environment, where the CPS deployment can be tested against real users and measurements allow to validate the proposal in a real world deployment:

- Sánchez-de-Rivera, D., Martín, D., Alcarria, R., Bordel, B., \& Robles, T. (2017). Towards a wireless and low-power infrastructure for representing information based on e-paper displays. Sustainability, 9(1), 76. JCR (2017): 2.075 Q2.

- Sánchez, B. B., Alcarria, R., Sánchez-Picot, Á., \& Sánchez-de-Rivera, D. (2017). A methodology for the design of application-specific cyber-physical social sensing cosimulators. Sensors, 17(10), 2177. JCR (2017) 2.475 Q2.

- Alcarria, R., de Andrés, D. M., Bordel, B., Sánchez de Rivera, D., Sánchez-Picot, Á., \& Robles, T. (2017, January). A service-oriented monitoring system based on rule evaluation for Home Automation. In 2017 IEEE International Conference on Consumer Electronics (ICCE) (pp. 329-330). IEEE.

- Martín, D., Alcarria, R., Sánchez-Picot, A., Robles, T., \& Sánchez de Rivera, D. (2014, December). A four-leaf clover shape methodology for prosumer service developments. In International Conference on Ubiquitous Computing and Ambient Intelligence (pp. 488-495). Springer, Cham.

- Bordel, B., Alcarria, R., Sánchez-Picot, Á., \& Sánchez-de-Rivera, D. (2019, February). Cyber-Physical Systems for Environment and People Monitoring in Large 
Facilities: A Study Case in Public Health. In International Conference on Information Technology \& Systems (pp. 406-416). Springer, Cham.

- Martín, D., Bordel, B., Alcarria, R., Sánchez-Picot, Á., Sánchez de Rivera, D., \& Robles, T. (2016, November). Improving learning tasks for mentally handicapped people using AmI environments based on cyber-physical systems. In International Conference on Ubiquitous Computing and Ambient Intelligence (pp. 166-177). Springer, Cham.

\section{Other publications by the author, indirectly related to this thesis:}

International peer reviewed Journals indexed in JCR:

- Alcarria, R., Bordel, B., Martín, D., \& Sánchez De Rivera, D. (2017). Rule-based monitoring and coordination of resource consumption in smart communities. IEEE Transactions on Consumer Electronics, 63(2), 191-199. JCR (2017): 1.802 Q3.

- Bordel, B., Orúe, A. B., Alcarria, R., \& Sánchez-De-Rivera, D. (2018). An intra-slice security solution for emerging $5 G$ networks based on pseudo-random number generators. IEEE Access, 6, 16149-16164. JCR (2017): 3.557 Q1.

- Sánchez-Picot, Á., Martín, D., Bordel, B., \& Sánchez-de-Rivera, D. (2018). AmI environments simulations approach integrating social and network aspects: A case study. Journal of Ambient Intelligence and Smart Environments, 10(4), 303-314. JCR (2017): 0.878 Q4.

International peer-reviewed Journals non-indexed in JCR:

- Sánchez de Rivera, D., \& Sánchez-Picot, A. (2015). Dynamic price generation and distribution system using ECA rules and ink labeling. IT CoNvergence PRActice (INPRA), 3(1), 31-41.

- Sánchez, B. B., Sánchez de Rivera, D., \& Sánchez-Picot, A. (2016). Building unobtrusive wearable devices: an ergonomic cybernetic glove. J. Internet Serv. Inf. Secur., 6(2), 37-52.

- Bordel Sánchez, B., Pérez Jiménez, M., \& Sánchez de Rivera, D. (2017). Recognition of activities of daily living in Enhanced Living Environments. IT CoNvergence PRActice (INPRA), 4(4), 18-31.

- Sánchez-Picot, A., \& Sánchez de Rivera, D. (2015). Collaboration between services using a B2B Platform in the retail environment. IT CoNvergence PRActice (INPRA), $3(2), 36-45$.

International peer-reviewed conferences with proceedings:

- Martín, D., Bordel, B., Alcarria, R., Sánchez-Picot, Á., Sánchez de Rivera, D., \& Robles, T. (2017, November). Prosumerization Approach to Semantic Ambient Intelligence Platforms. In International Conference on Ubiquitous Computing and Ambient Intelligence (pp. 109-120). Springer, Cham.

- Bordel, B., Alcarria, R., Sánchez-de-Rivera, D., \& Robles, T. (2017, November). Protecting industry 4.0 systems against the malicious effects of cyber-physical attacks. In 
International Conference on Ubiquitous Computing and Ambient Intelligence (pp. 161-171). Springer, Cham.

- Sánchez-Picot, Á., Martín, D., Sánchez de Rivera, D., Bordel, B., \& Robles, T. (2016, March). Modeling and simulation of interactions among people and devices in ambient intelligence environments. In 2016 30th International Conference on Advanced Information Networking and Applications Workshops (WAINA) (pp. 784-789). IEEE.

- Bordel, B., Alcarria, R., \& Sánchez-de-Rivera, D. (2017, April). Detecting malicious components in large-scale Internet-of-Things systems and architectures. In World Conference on Information Systems and Technologies (pp. 155-165). Springer, Cham.

- Sánchez-Picot, Á., Sánchez-de-Rivera, D., Robles, T., \& Jiménez, J. (2018, June). Time Analysis of the Integration of Simulators for an AmI Environment. In International Symposium on Distributed Computing and Artificial Intelligence (pp. 157-164). Springer, Cham.

- Robles, T., Bordel, B., Alcarria, R., \& Sánchez-de-Rivera, D. (2018). Blockchain Technologies for Private Data Management in AmI Environments. In Multidisciplinary Digital Publishing Institute Proceedings (Vol. 2, No. 19, p. 1230).

- Bordel, B., Alcarria, R., Sánchez-de-Rivera, D., \& Sánchez, Á. (2019, March). An Inter-slice Management Solution for Future Virtualization-Based 5G Systems. In International Conference on Advanced Information Networking and Applications (pp. 1059-1070). Springer, Cham.

- Bordel, B., Alcarria, R., Martín, D., \& Sánchez-de-Rivera, D. (2017, May). Improving MOOC student learning through enhanced peer-to-peer tasks. In European Conference on Massive Open Online Courses (pp. 140-149). Springer, Cham.

- Bordel, B., Sánchez de Rivera, D., \& Alcarria, R. (2018, March). Virtualization-Based Techniques for the Design, Management and Implementation of Future 5G Systems with Network Slicing. In World Conference on Information Systems and Technologies (pp. 133-143). Springer, Cham.

To summarize, Table 16 shows the publications number achieved in this $\mathrm{PhD}$ Thesis work.

Table 16. Achieved publications

\begin{tabular}{|c|c|c|c|}
\hline & JCR Indexed & $\begin{array}{c}\text { Non indexed } \\
\text { Journal }\end{array}$ & $\begin{array}{c}\text { Conference } \\
\text { proceedings }\end{array}$ \\
\hline $\begin{array}{c}\text { Directly } \\
\text { related }\end{array}$ & 8 & - & 18 \\
\hline $\begin{array}{c}\text { Indirectly } \\
\text { related }\end{array}$ & 3 & 3 & 9 \\
\hline Total & $\mathbf{1 1}$ & $\mathbf{3}$ & $\mathbf{2 7}$ \\
\hline
\end{tabular}




\section{References}

Aberer, K., Hauswirth, M., \& Salehi, A. (2006). The Global Sensor Networks middleware for efficient and flexible deployment and interconnection of sensor networks. Retrieved from https://infoscience.epfl.ch/record/83891

Alcarria, R., Bordel, B., Robles, T., Martín, D., \& Manso-Callejo, M.-Á. (2018). A Blockchain-Based Authorization System for Trustworthy Resource Monitoring and Trading in Smart Communities. Sensors (Basel, Switzerland), 18(10). https://doi.org/10.3390/s18103561

Alcarria, R., Robles, T., Morales, A., \& González-Miranda, S. (2012). New Service Development Method for Prosumer Environments. 6.

Alcarria, R., Robles, T., Morales, A., López-de-Ipiña, D., \& Aguilera, U. (2012). Enabling Flexible and Continuous Capability Invocation in Mobile Prosumer Environments. Sensors (Basel, Switzerland), 12(7), 8930-8954. https://doi.org/10.3390/s120708930

Al-Khawaldeh, M., Al-Naimi, I., Chen, X., \& Moore, P. (2016). Ubiquitous robotics for knowledge-based auto-configuration system within smart home environment. 2016 7th International Conference on Information and Communication Systems (ICICS), 139-144. https://doi.org/10.1109/IACS.2016.7476100

Appel, S., Frischbier, S., Freudenreich, T., \& Buchmann, A. (2013). Event Stream Processing Units in Business Processes. In F. Daniel, J. Wang, \& B. Weber (Eds.), Business Process Management (pp. 187-202). Springer Berlin Heidelberg.

AutoPnP. (n.d.). Retrieved 19 March 2019, from http://www.autopnp.com/

Bicer, V., Kilic, O., Dogac, A., \& Laleci, G. B. (2005). Archetype-Based Semantic Interoperability of Web Service Messages in the Health Care Domain. 
International Journal on Semantic Web and Information Systems (IJSWIS), 1(4), 1-23. https://doi.org/10.4018/jswis.2005100101

Bonzini, P., \& Pozzi, L. (2006). Code transformation strategies for extensible embedded processors. In S. Hong, W. H. Wolf, K. Flautner, \& T. Kim (Eds.), Proceedings of the 2006 International Conference on Compilers, Architecture, and Synthesis for Embedded Systems, CASES 2006, Seoul, Korea, October 22-25, 2006 (pp. 242-252). https://doi.org/10.1145/1176760.1176791

Bordel, B., Iturrioz, T., Alcarria, R., \& Sanchez-Picot, A. (2018). Provision of nextgeneration personalized cyber-physical services. 2018 13th Iberian Conference on Information Systems and Technologies (CISTI), 1-6. https://doi.org/10.23919/CISTI.2018.8399338

Bordel, Borja, Alcarria, R., Robles, T., \& Martín, D. (2017). Cyber-physical systems: Extending pervasive sensing from control theory to the Internet of Things. Pervasive and Mobile Computing, 40, 156-184. https://doi.org/10.1016/j.pmcj.2017.06.011

Bordel, Borja, Alcarria, R., Sánchez de Rivera, D., Martín, D., \& Robles, T. (2018). Fast self-configuration in service-oriented Smart Environments for real-time applications. Journal of Ambient Intelligence and Smart Environments, 10(2), 143167. https://doi.org/10.3233/AIS-180479

Bordel, Borja, Miguel, C., Alcarria, R., \& Robles, T. (2018). A Hardware-Supported Algorithm for Self-Managed and Choreographed Task Execution in Sensor Networks. Sensors (Basel, Switzerland), 18(3). https://doi.org/10.3390/s18030812

Bordel Sánchez, B., Alcarria, R., Martín, D., \& Robles, T. (2015). TF4SM: A Framework for Developing Traceability Solutions in Small Manufacturing Companies. Sensors (Basel, $\quad$ Switzerland), 15(11), 29478-29510. https://doi.org/10.3390/s151129478

Byun, J., Jeon, B., Noh, J., Kim, Y., \& Park, S. (2012). An intelligent self-adjusting sensor for smart home services based on ZigBee communications. IEEE Transactions on Consumer Electronics, 58(3), 794-802. https://doi.org/10.1109/TCE.2012.6311320

Cetina, C., Giner, P., Fons, J., \& Pelechano, V. (2009). Using Feature Models for Developing Self-Configuring Smart Homes. 2009 Fifth International Conference on 
Autonomic and Autonomous 179-188. https://doi.org/10.1109/ICAS.2009.50

Charif, Y., \& Sabouret, N. (2006). An Overview of Semantic Web Services Composition Approaches. Electronic Notes in Theoretical Computer Science, 146(1), 33-41. https://doi.org/10.1016/j.entcs.2005.11.005

Chen, T., Xiao, N., \& Liu, F. (2010). Multi-aggregate-query Scheduling over Data Streams. 2010 International Conference on Parallel and Distributed Computing, Applications and Technologies, 27-33. https://doi.org/10.1109/PDCAT.2010.92

Chidlovskii, B., \& Borghoff, U. M. (1998). Query translation for distributed information gathering on the Web. Proceedings. IDEAS'98. International Database Engineering and Applications Symposium (Cat. No.98EX156), 214-223. https://doi.org/10.1109/IDEAS.1998.694381

Childers, B., Davidson, J. W., \& Soffa, M. L. (2003). Continuous compilation: a new approach to aggressive and adaptive code transformation. Proceedings International Parallel and Distributed Processing Symposium, 10. https://doi.org/10.1109/IPDPS.2003.1213375

Clauß, S., \& Schulte, A. (2014). Task delegation in an agent supervisory control relationship capability awareness in a cognitive agent. 2014 IEEE International Conference on Systems, Man, and Cybernetics (SMC), 825-830. https://doi.org/10.1109/SMC.2014.6974013

Colombo, A.-W., Karnouskos, S., \& Mendes, J.-M. (2010). Factory of the Future: A Service-oriented System of Modular, Dynamic Reconfigurable and Collaborative Systems. In L. Benyoucef \& B. Grabot (Eds.), Artificial Intelligence Techniques for Networked Manufacturing Enterprises Management (pp. 459-481). https://doi.org/10.1007/978-1-84996-119-6_15

Cook, D., \& Das, S. (2004). Smart Environments: Technology, Protocols and Applications (Wiley Series on Parallel and Distributed Computing). New York, NY, USA: WileyInterscience.

Cook, D. J., \& Das, S. K. (2007). How smart are our environments? An updated look at the state of the art. Pervasive and Mobile Computing, 3(2), 53-73. https://doi.org/10.1016/j.pmcj.2006.12.001 
Crisan, V., \& Rantzau, R. (2008). An alert notification facility for RFID event repositories. 2008 IEEE 24th International Conference on Data Engineering Workshop, 88-93. https://doi.org/10.1109/ICDEW.2008.4498292

Czarnecki, K., \& Helsen, S. (2003). Classification of Model Transformation Approaches.

Dawy, Z., Saad, W., Ghosh, A., Andrews, J. G., \& Yaacoub, E. (2017). Toward Massive Machine Type Cellular Communications. IEEE Wireless Communications, 24(1), 120-128. https://doi.org/10.1109/MWC.2016.1500284WC

De, D., Song, W., Xu, M., Wang, C., Cook, D., \& Huo, X. (2012). FindingHuMo: RealTime Tracking of Motion Trajectories from Anonymous Binary Sensing in Smart Environments. 2012 IEEE 32nd International Conference on Distributed Computing Systems, 163-172. https://doi.org/10.1109/ICDCS.2012.76

Derler, P., Lee, E. A., Tripakis, S., \& Törngren, M. (2013). Cyber-physical System Design Contracts. Proceedings of the ACM/IEEE 4th International Conference on CyberPhysical Systems, 109-118. https://doi.org/10.1145/2502524.2502540

Dillon, T., Potdar, V., Singh, J., \& Talevski, A. (2011). Cyber-physical systems: Providing Quality of Service (QoS) in a heterogeneous systems-of-systems environment. 5th IEEE International Conference on Digital Ecosystems and Technologies (IEEE DEST 2011), 330-335. https://doi.org/10.1109/DEST.2011.5936595

Ding, F., Song, A., Tong, E., \& Li, J. (2016). A Smart Gateway Architecture for Improving Efficiency of Home Network Applications [Research article]. https://doi.org/10.1155/2016/2197237

Erradi, A., Maheshwari, P., \& Tosic, V. (2006). Policy-Driven Middleware for Selfadaptation of Web Services Compositions. In M. van Steen \& M. Henning (Eds.), Middleware 2006 (pp. 62-80). Springer Berlin Heidelberg.

EuroCPS I Cyber-Physical Systems. (n.d.). Retrieved 23 January 2019, from https://www.eurocps.org/

Evensen, P., \& Meling, H. (2009). SenseWrap: A service oriented middleware with sensor virtualization and self-configuration. 2009 International Conference on Intelligent Sensors, Sensor Networks and Information Processing (ISSNIP), 261-266. https://doi.org/10.1109/ISSNIP.2009.5416827 
Familiar, M. S., Martínez, J. F., Corredor, I., \& García-Rubio, C. (2012). Building Serviceoriented Smart Infrastructures over Wireless Ad Hoc Sensor Networks: A Middleware Perspective. Comput. Netw., 56(4), 1303-1328. https://doi.org/10.1016/j.comnet.2011.12.005

Familiar, M. S., Martínez, J. F., \& López, L. (2012). Pervasive Smart Spaces and Environments: A Service-Oriented Middleware Architecture for Wireless Ad Hoc and Sensor Networks. International Journal of Distributed Sensor Networks, 8(4), 725190. https://doi.org/10.1155/2012/725190

Gannon, and D. (2006). A comparative study of Web services-based event notification specifications. 2006 International Conference on Parallel Processing Workshops (ICPPW'06), 8 pp. - 14. https://doi.org/10.1109/ICPPW.2006.5

Gouin-Vallerand, C., Abdulrazak, B., Giroux, S., \& Dey, A. K. (2013). A Context-aware Service Provision System for Smart Environments Based on the User Interaction Modalities. J. Ambient Intell. Smart Environ., 5(1), 47-64.

Houyou, A. M. (2012). Internet of things at work: Architecture approach to interoperability. Presentation Slide at IoT Interoperability Workshop Paris, March.

Hu, H., Zhang, J., Zheng, X., Yang, Y., \& Wu, P. (2010). Self-configuration and selfoptimization for LTE networks. IEEE Communications Magazine, 48(2), 94-100. https://doi.org/10.1109/MCOM.2010.5402670

Jammes, F., Karnouskos, S., Bony, B., Nappey, P., Colombo, A. W., Delsing, J., ... Bangemann, T. (2014). Promising Technologies for SOA-Based Industrial Automation Systems. In A. W. Colombo, T. Bangemann, S. Karnouskos, J. Delsing, P. Stluka, R. Harrison, ... J. L. Lastra (Eds.), Industrial Cloud-Based CyberPhysical Systems: The IMC-AESOP Approach (pp. 89-109). https://doi.org/10.1007/978-3-319-05624-1_4

Karlan, D., \& Zinman, J. (2018). Price and control elasticities of demand for savings. Journal of Development Economics, 130(C), 145-159.

Kim, J., Lakshmanan, K., \& Rajkumar, R. (Raj). (2012). Rhythmic Tasks: A New Task Model with Continually Varying Periods for Cyber-Physical Systems. Proceedings of the 2012 IEEE/ACM Third International Conference on Cyber-Physical Systems, 5564. https://doi.org/10.1109/ICCPS.2012.14 
Kimura, N., \& Latifi, S. (2005). A survey on data compression in wireless sensor networks. International Conference on Information Technology: Coding and Computing (ITCC'05) - Volume II, 2, $\quad 8-13 \quad$ Vol. 2. https://doi.org/10.1109/ITCC.2005.43

Klie, T., Fischer, S., \& Mueller, F. (2011). Network Monitoring with Asynchronous Notifications in Web Service Environments.

Kortuem, G., Kawsar, F., Sundramoorthy, V., \& Fitton, D. (2010). Smart objects as building blocks for the Internet of things. IEEE Internet Computing, 14(1), 44-51. https://doi.org/10.1109/MIC.2009.143

Kumar, A., \& Singh, B. (2015). Optimizing compression for power efficient wireless multimedia sensor networks. 2015 IEEE International Conference on Computational Intelligence and Computing Research (ICCIC), 1-4. https://doi.org/10.1109/ICCIC.2015.7435776

Kyusakov, R., Eliasson, J., Delsing, J., Deventer, J. van, \& Gustafsson, J. (2013). Integration of Wireless Sensor and Actuator Nodes With IT Infrastructure Using Service-Oriented Architecture. IEEE Transactions on Industrial Informatics, 9(1), 43-51. https://doi.org/10.1109/TII.2012.2198655

La, H. J., \& Kim, S. D. (2010). A Service-Based Approach to Designing Cyber Physical Systems. 2010 IEEE/ACIS 9th International Conference on Computer and Information Science, 895-900. https://doi.org/10.1109/ICIS.2010.73

Lai, C.-F., Ma, Y.-W., Chang, S.-Y., Chao, H.-C., \& Huang, Y.-M. (2011). OSGi-based services architecture for Cyber-Physical Home Control Systems. Computer Communications, 34(2), 184-191. https://doi.org/10.1016/j.comcom.2010.03.034

Lee, E. (2006). Cyber-Physical Systems - Are Computing Foundations Adequate? Presented at the Position Paper for NSF Workshop On Cyber-Physical Systems: Research Motivation, Techniques and Roadmap. Retrieved from http://chess.eecs.berkeley.edu/pubs/329.html

Lee, J., Dong, M., \& Sun, Y. (2015). A preliminary study of low power wireless technologies: ZigBee and Bluetooth Low Energy. 2015 IEEE 10th Conference on Industrial Electronics and Applications (ICIEA), 135-139. https://doi.org/10.1109/ICIEA.2015.7334098 
Lee, M., Kim, Y., \& Lee, Y. (2015). A home cloud-based home network auto-configuration using SDN. 2015 IEEE 12th International Conference on Networking, Sensing and Control, 444-449. https://doi.org/10.1109/ICNSC.2015.7116078

Lee, S., Jeong, J. P., \& Park, J. (2016). DNSNA: DNS name autoconfiguration for Internet of Things devices. 2016 18th International Conference on Advanced Communication Technology (ICACT), 410-416. https://doi.org/10.1109/ICACT.2016.7423412

Lin, H., \& Bergmann, N. W. (2016). IoT Privacy and Security Challenges for Smart Home Environments. Information, 7(3), 44. https://doi.org/10.3390/info7030044

Lin, K., \& Panahi, M. (2010). A real-time service-oriented framework to support sustainable cyber-physical systems. 2010 8th IEEE International Conference on Industrial Informatics, 15-21. https://doi.org/10.1109/INDIN.2010.5549473

Loyall, J. P., Gillen, M., Haigh, K. Z., Walsh, R., Partridge, C., Lauer, G., \& Strayer, T. (2012). A concept for publish-subscribe information dissemination and networking. 2012 IEEE International Conference on Communications (ICC), 58105816. https://doi.org/10.1109/ICC.2012.6364730

Ma, T., Hempel, M., Peng, D., \& Sharif, H. (2013). A Survey of Energy-Efficient Compression and Communication Techniques for Multimedia in Resource Constrained Systems. IEEE Communications Surveys Tutorials, 15(3), 963-972. https://doi.org/10.1109/SURV.2012.060912.00149

Marrella, A., \& Mecella, M. (2017). Adaptive Process Management in Cyber-Physical Domains. In G. Grambow, R. Oberhauser, \& M. Reichert (Eds.), Advances in Intelligent Process-Aware Information Systems: Concepts, Methods, and Technologies (pp. 15-48). https://doi.org/10.1007/978-3-319-52181-7_2

Mayer, S., Verborgh, R., Kovatsch, M., \& Mattern, F. (2016). Smart Configuration of Smart Environments. IEEE Transactions on Automation Science and Engineering, 13(3), 1247-1255. https://doi.org/10.1109/TASE.2016.2533321

Moldovan, D., Copil, G., \& Dustdar, S. (2018). Elastic systems: Towards cyber-physical ecosystems of people, processes, and things. Computer Standards $\mathcal{E}$ Interfaces, 57, 76-82. https://doi.org/10.1016/j.csi.2017.04.002

Mondal, N. I., Zaman, S. U., Masud, A. A., \& Alam, J. (2008). Comparisons of maximum system lifetime in diverse scenarios for body sensor networks. 2008 11th 
International Conference on Computer and Information Technology, 73-78. https://doi.org/10.1109/ICCITECHN.2008.4803006

Mönks, U., Trsek, H., Dürkop, L., Geneiß, V., \& Lohweg, V. (2014). Assisting the Design of Sensor and Information Fusion Systems. Procedia Technology, 15, 35-45. https://doi.org/10.1016/j.protcy.2014.09.032

Morales, A., Robles, T., Alcarria, R., \& Cedeño, E. (2013). On the Support of Scientific Workflows over Pub/Sub Brokers. Sensors (Basel, Switzerland), 13(8), 10954-10980. https://doi.org/10.3390/s130810954

Mueller, M. (2015). Reducing Hazards in Multiagent Task Delegation. Wirtschaftsinformatik Proceedings 2015. Retrieved from https://aisel.aisnet.org/wi2015/112

Munir, S., Stankovic, J. A., Liang, C.-J. M., \& Lin, S. (2013). Cyber Physical System Challenges for Human-in-the-Loop Control. Presented at the Presented as part of the 8th International Workshop on Feedback Computing. Retrieved from https://www.usenix.org/node/174694

Nair, K., Kulkarni, J., Warde, M., Dave, Z., Rawalgaonkar, V., Gore, G., \& Joshi, J. (2015). Optimizing power consumption in iot based wireless sensor networks using Bluetooth Low Energy. 2015 International Conference on Green Computing and Internet of Things (ICGCIoT), 589-593. https://doi.org/10.1109/ICGCIoT.2015.7380533

Nimmagadda, Y., Kumar, K., \& Lu, Y. (2009). Energy-efficient image compression in mobile devices for wireless transmission. 2009 IEEE International Conference on Multimedia and Expo, 1278-1281. https://doi.org/10.1109/ICME.2009.5202735

nsnam. (n.d.). ns-3. Retrieved 26 March 2019, from ns-3 website: /

OpenSCADA: Main. (n.d.). Retrieved 26 March 2019, from http://oscada.org/

Pereira, E. G., \& Pereira, R. (2015). Video Encoding and Streaming Mechanisms in IoT Low Power Networks. 2015 3rd International Conference on Future Internet of Things and Cloud, 357-362. https://doi.org/10.1109/FiCloud.2015.88

Puthenpurayil, S., Gu, R., \& Bhattacharyya, S. S. (2007). Energy-Aware Data Compression for Wireless Sensor Networks. 2007 IEEE International Conference on 
Acoustics, Speech and Signal Processing - ICASSP '07, 2, II-45-II-48. https://doi.org/10.1109/ICASSP.2007.366168

Raghunathan, V., Schurgers, C., \& Srivastava, and M. B. (2002). Energy-aware wireless microsensor networks. IEEE Signal Processing Magazine, 19(2), 40-50. https://doi.org/10.1109/79.985679

Rajkumar, R., Lee, I., Sha, L., \& Stankovic, J. (2010). Cyber-physical systems: The next computing revolution. Design Automation Conference, 731-736. https://doi.org/10.1145/1837274.1837461

Rivera, D. S. de, Alcarria, R., Andres, D. M. de, Bordel, B., \& Robles, T. (2016). An autonomous information device with e-paper display for personal environments. 2016 IEEE International Conference on Consumer Electronics (ICCE), 139-140. https://doi.org/10.1109/ICCE.2016.7430554

Robles, T., Alcarria, R., Andrés, D. M. de, Cruz, M. N. de la, Calero, R., Iglesias, S., \& López, M. (2015). An IoT based reference architecture for smart water management processes. JoWUA, 6, 4-23.

Rogers, J. A., Bao, Z., Baldwin, K., Dodabalapur, A., Crone, B., Raju, V. R., ... Drzaic, P. (2001). Paper-like electronic displays: Large-area rubber-stamped plastic sheets of electronics and microencapsulated electrophoretic inks. Proceedings of the National Academy of Sciences, 98(9), 4835-4840. https://doi.org/10.1073/pnas.091588098

Rust, P., Picard, G., \& Ramparany, F. (2016). Using Message-passing DCOP Algorithms to Solve Energy-efficient Smart Environment Configuration Problems. Proceedings of the Twenty-Fifth International Joint Conference on Artificial Intelligence, 468-474. Retrieved from http://dl.acm.org/citation.cfm?id=3060621.3060687

Sánchez-de-Rivera, D., Alcarria, R., Martín, D., \& Huecas, G. (2018). Proposal and Assessment of Algorithms for Power Consumption Reduction in Wireless Networks with E-Ink Displays. (19:7), 2035-2046.

Seiger, R., Huber, S., \& Schlegel, T. (2018). Toward an execution system for self-healing workflows in cyber-physical systems. Software E Systems Modeling, 17(2), 551572. https://doi.org/10.1007/s10270-016-0551-z 
Sen, S. (2016). Invited - Context-aware Energy-efficient Communication for IoT Sensor Nodes. Proceedings of the 53rd Annual Design Automation Conference, 67:1-67:6. https://doi.org/10.1145/2897937.2905005

Sharma, R. (2015). A data compression application for wireless sensor networks using LTC algorithm. 2015 IEEE International Conference on Electro/Information Technology (EIT), 598-604. https://doi.org/10.1109/EIT.2015.7293435

Shon, T., \& Park, Y. (2010). Implementation of RF4CE-Based Wireless Auto Configuration Architecture for Ubiquitous Smart Home. 2010 International Conference on Complex, Intelligent and Software Intensive Systems, 779-783. https://doi.org/10.1109/CISIS.2010.36

Smirnov, A., Kashevnik, A., Shilov, N., Makklya, A., \& Gusikhin, O. (2013). Contextaware service composition in cyber physical human system for transportation safety. 2013 13th International Conference on ITS Telecommunications (ITST), 139144. https://doi.org/10.1109/ITST.2013.6685535

Sridhar, S., Hahn, A., \& Govindarasu, M. (2012). Cyber-Physical System Security for the Electric Power Grid. Proceedings of the IEEE, 100(1), 210-224. https://doi.org/10.1109/JPROC.2011.2165269

Study Report on IoT Reference Architectures/Frameworks - PDF. (n.d.). Retrieved 23 January 2019, from https://docplayer.net/16351625-Study-report-on-iotreference-architectures-frameworks.html

Sungur, C. T., Spiess, P., Oertel, N., \& Kopp, O. (2013). Extending BPMN for Wireless Sensor Networks. 2013 IEEE 15th Conference on Business Informatics, 109-116. https://doi.org/10.1109/CBI.2013.24

Szalapski, T., \& Madria, S. (2014). Toward energy efficient multistream collaborative compression in wireless sensor networks. 10th IEEE International Conference on Collaborative Computing: Networking, Applications and Worksharing, 124-133.

Sztipanovits, J., Koutsoukos, X., Karsai, G., Kottenstette, N., Antsaklis, P., Gupta, V., ... Wang, S. (2012). Toward a Science of Cyber-Physical System Integration. Proceedings of the IEEE, 100(1), 29-44. https://doi.org/10.1109/JPROC.2011.2161529

Tamarit, S., Mariño, J., Vigueras, G., \& Carro, M. (2017). Towards a Semantics-Aware Code Transformation Toolchain for Heterogeneous Systems. Electronic 
Proceedings in Theoretical Computer Science, 237, 34-51. https://doi.org/10.4204/EPTCS.237.3

Tan, Y., Goddard, S., \& Pérez, L. C. (2008). A Prototype Architecture for Cyber-physical Systems. SIGBED Rev., 5(1), 26:1-26:2. https://doi.org/10.1145/1366283.1366309

The Official YAML Web Site. (n.d.). Retrieved 26 March 2019, from https://yaml.org/

Thompson, K. D. (2014, June 20). Cyber-Physical Systems. Retrieved 23 January 2019, from NIST website: https://www.nist.gov/el/cyber-physical-systems

TRAN, V. X., \& TSUJI, H. (2007). OWL-T: A Task Ontology Language for Automatic Service Composition. IEEE International Conference on Web Services (ICWS 2007), 1164-1167. https://doi.org/10.1109/ICWS.2007.138

Tranquillini, S., Spieß, P., Daniel, F., Karnouskos, S., Casati, F., Oertel, N., ... Voigt, T. (2012). Process-Based Design and Integration of Wireless Sensor Network Applications. In A. Barros, A. Gal, \& E. Kindler (Eds.), Business Process Management (pp. 134-149). Springer Berlin Heidelberg.

Vimercati, S. D. C. di, Foresti, S., Jajodia, S., Livraga, G., Paraboschi, S., \& Samarati, P. (2014). Integrity for distributed queries. 2014 IEEE Conference on Communications and Network Security, 364-372. https://doi.org/10.1109/CNS.2014.6997505

Wan, J., Zhang, D., Zhao, S., Yang, L. T., \& Lloret, J. (2014). Context-aware vehicular cyber-physical systems with cloud support: architecture, challenges, and solutions. IEEE Communications Magazine, 52(8), 106-113. https://doi.org/10.1109/MCOM.2014.6871677

Wan, Jiafu, Chen, M. D., Xia, F., Li, D., \& Zhou, K. (2013). W From Machine-to-Machine Communications towards Cyber-Physical Systems.

Wang, J., Zhu, Q., \& Ma, Y. (2013). An agent-based hybrid service delivery for coordinating internet of things and 3rd party service providers. Journal of Network and Computer Applications, 36(6), 1684-1695. https://doi.org/10.1016/j.jnca.2013.04.014

Wang, S., Wan, J., Li, D., \& Zhang, C. (2016). Implementing Smart Factory of Industrie 4.0: An Outlook. International Journal of Distributed Sensor Networks, 2016. Retrieved from https://doaj.org 
Youngblood, G. M., Cook, D. J., \& Holder, L. B. (2005). Managing Adaptive Versatile environments. Pervasive and Mobile Computing, 1(4), 373-403. https://doi.org/10.1016/j.pmcj.2005.08.004

Youngblood, G. M., Heierman, E. O., Holder, L. B., \& Cook, D. J. (2005). Automation Intelligence for the Smart Environment. Proceedings of the 19th International Joint Conference on Artificial Intelligence, 1513-1514. Retrieved from http://dl.acm.org/citation.cfm?id=1642293.1642535

Zeng, L., Benatallah, B., Dumas, M., Kalagnanam, J., \& Sheng, Q. Z. (2003). Quality Driven Web Services Composition. Proceedings of the 12th International Conference on World Wide Web, 411-421. https://doi.org/10.1145/775152.775211

Zhang, F., Szwaykowska, K., Wolf, W., \& Mooney, V. (2008). Task Scheduling for Control Oriented Requirements for Cyber-Physical Systems. 2008 Real-Time Systems Symposium, 47-56. https://doi.org/10.1109/RTSS.2008.52 